\title{
First-order Complete and Computationally Complete Query Languages for Spatio-Temporal Databases ${ }^{1}$
}

\author{
Floris Geerts ${ }^{2}$, Sofie Haesevoets, Bart Kuijpers ${ }^{3}$ \\ University of Limburg
}

\begin{abstract}
We address a fundamental question concerning spatio-temporal database systems: "What are exactly spatio-temporal queries?" We define spatio-temporal queries to be computable mappings that are also generic, meaning that the result of a query may only depend to a limited extent on the actual internal representation of the spatio-temporal data. Genericity is defined as invariance under groups of geometric transformations that preserve certain characteristics of spatio-temporal data (e.g., collinearity, distance, velocity, acceleration, ...). These groups depend on the notions that are relevant in particular spatio-temporal database applications. These transformations also have the distinctive property that they respect the monotone and unidirectional nature of time.

We investigate different genericity classes with respect to the constraint database model for spatio-temporal databases and we identify sound and complete languages for the first-order and the computable queries in these genericity classes. We distinguish between genericity determined by time-invariant transformations, genericity notions concerning physical quantities and genericity determined by time-dependent transformations.
\end{abstract}

Categories and Subject Descriptors: []:

General Terms:

Additional Key Words and Phrases:

\section{INTRODUCTION}

Since the early 1990s, various database systems have been developed to handle spatial data [Abel and Ooi 1993; Buchmann 1989; Egenhofer and Herring 1995; Gunther and Schek 1991; Güting 1999; Rigaux et al. 2000; Scholl and Voisard 1997] and solid theories for such systems have been proposed and studied [Paredaens et al. 1994; Kuper and Scholl 2000]. Conceptually, spatial databases contain possibly infinite sets of points in a real space $\mathbf{R}^{n}$. In more recent years, we have seen the emergence of database systems and applications that are dealing with spatiotemporal data [Böhlen et al. 1999; Chen and Zaniolo 2000; Frank et al. 1999; Güting et al. 2000; Pfoser and Tryfona 1998]. Conceptually, spatio-temporal data can be modeled as infinite spatial sets that move or change in time, i.e., sets in $\mathbf{R}^{n} \times \mathbf{R}$.

A recent and much acclaimed method for effectively representing infinite geometrical figures is provided by the constraint database model, that was introduced in 1990 by Kanellakis, Kuper and Revesz [Kanellakis et al. 1990; 1995] (an overview of the area of constraint databases appeared [Paredaens et al. 2000]; and [Revesz

\footnotetext{
${ }^{1}$ An extended abstract appeared in the proceedings of DBPL'01 [Geerts et al. 2001].

${ }^{2}$ Post-doctoral researcher of the FWO-Vlaanderen and visiting researcher at the University of Edinburgh.

${ }^{3}$ Corresponding author: University of Limburg, Department of Mathematics, Physics and Computer Science, B-3590 Diepenbeek, Belgium, bart.kuijpers@luc.ac.be
} 


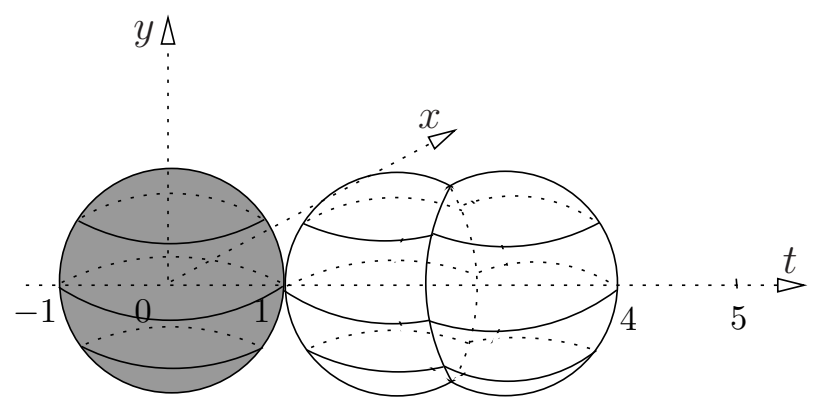

Fig. 1. An example of a spatio-temporal database in $\mathbf{R}^{2} \times \mathbf{R}$.

2002] is a textbook on this topic). Until recently this model has been used mainly in the area of spatial databases, but it provides an equally elegant and efficient way to model spatio-temporal data [Chen and Zaniolo 2000; Chomicki and Revesz 1997; 1999; Grumbach et al. 1998; Kuijpers et al. 2000]. In the setting of the constraint model, a spatio-temporal relation in $\mathbf{R}^{n} \times \mathbf{R}$ is finitely represented as a Boolean combination of polynomial equalities and inequalities. Figure 1 depicts the spatio-temporal database $\left\{(x, y ; t) \mid x^{2}+y^{2}+t^{2} \leq 1 \vee\left(x^{2}+y^{2}+(t-2)^{2}=\right.\right.$ $\left.1 \wedge t \leq 5 / 2) \vee\left(x^{2}+y^{2}+(t-3)^{2}=1 \wedge t>5 / 2\right)\right\}$ in $\mathbf{R}^{2} \times \mathbf{R}$. A spatio-temporal database is a finite collection of such relations and can be finitely represented by the polynomial constraint formulas that represent its relations.

A number of theoretical studies have appeared on the status of time and its relation with space in systems that model moving objects. Erwig et al. [Erwig et al. 1999] give a taxonomy of applications ranging from those that rely on a step-wise constant geometry to applications which need more complete integration of space and time (like for instance a continuous description of a trajectory). MOST [Sistla et al. 1997], an example of the latter category, relies on a strong interaction of the space and time components (since the space variables are described by linear polynomials in time) and provides a query language that is a combination of a spatial query language and a temporal logic. On the other range of the spectrum, variable independence (defined in terms of orthographic dimension) gives rise to a less expressive data model which has the advantage of a lower complexity of query evaluation [Grumbach et al. 1998; Libkin 2000].

We study spatio-temporal queries from the perspective of expressive power, and do this against the background of the full modeling and querying power of the constraint database model and the first-order and computationally complete languages it offers. We ask which expressions in these languages may be considered as reasonable spatio-temporal queries. In database theory it is usually required that the result of queries should only to a certain limited extent depend on the actual internal representation of databases and that queries should only ask for properties that are shared by "isomorphic" encodings of the same data. The meaning of "isomorphic" may be influenced by the actual database application and by which notions are relevant to it. In the context of the relational database model, Chandra and Harel [Chandra and Harel 1980] formalized this independence of the actual encoding in terms of the notion of genericity. Paredaens, Van den Bussche and Van 
Gucht [Paredaens et al. 1994] identified a hierarchy of genericity classes for spatial database applications. The generic queries in the different classes focus on different geometrical and topological aspects of the spatial data. On a technical level, generic queries are defined as being invariant under those transformations of the data that preserve the relevant aspects of the data. Whereas Chandra and Harel considered the group of the isomorphisms (that possibly fix some elements of the domain) in the case of relational databases, Paredaens, Van den Bussche and Van Gucht identified different geometrical and topological transformation groups (affinities, isometries, translations, homeomorphisms, ...) for spatial database applications.

We define spatio-temporal queries to be computable mappings that are also generic, meaning that the result of a query may only depend to a limited extent on the actual internal representation of the spatio-temporal data. Genericity is defined as invariance under some (application-dependent) group of geometric transformations. These transformations preserve certain characteristics of spatio-temporal data (e.g., collinearity, distance, velocity, acceleration, ...).

We investigate which notions of genericity are appropriate for spatio-temporal databases and which transformation groups express them. We observe that the transformations should first and foremost respect the monotone and unidirectional nature of time, i.e., leave the temporal order of events unchanged. It follows that the relevant transformation groups are the product of a group of time-(in)dependent spatial transformations and a group of monotone increasing transformations of the time-component of the spatio-temporal data. Next, we focus on the former groups and study which of them leave different spatial and spatio-temporal properties (like collinearity, distance and orientation) unchanged. We also focus on physical properties of spatio-temporal data (like velocity and acceleration). The transformation groups that we consider are all subgroups of the time-dependent or time-independent affinities of $\mathbf{R}^{n} \times \mathbf{R}$.

We study the notion of spatio-temporal genericity relative to two popular query languages in the constraint model: first-order logic over the reals (FO) and an extension of this logic with a while-loop $(\mathrm{FO}+\mathrm{While})$. Both languages are known to be effectively computable (given termination in the case of FO + While-programs) and $\mathrm{FO}+$ While is known to be a computationally complete language on spatiotemporal databases [Van den Bussche 2000]. First, we show that all the genericity classes are undecidable. We show that the considered classes of generic first-order queries are recursively enumerable, however. Hereto, we define first-order pointbased languages in which variables are assumed to range over points in $\mathbf{R}^{n} \times \mathbf{R}$ and which contain certain point predicates (such as Between and Before). These point-based languages are shown to be sound and complete for the first-order queries in the considered genericity classes. We have also shown that extensions of these point-based logics with a While-loop give sound and complete languages for the computable queries in the different genericity classes. Our results are inspired by similar results that were obtained by Gyssens, Van den Bussche and Van Gucht in the context of spatial databases [Gyssens et al. 1999]. Also, the proof techniques we use for time-independent transformation groups, are generalisations of techniques introduced in those papers. However, our results for genericity notions described by time-dependent transformations require new proof techniques. 
This paper is organized as follows. In Section 2, we define spatio-temporal databases, spatio-temporal queries, and the constraint query languages FO and FO + While. In Section 3, we define a number of genericity notions. In Section 4, we present sound and complete first-order query languages for the different notions of genericity. In Section 5, we present sound and complete languages for the computable queries satisfying the different notions of genericity. We end with a discussion in Section 6.

\section{DEFINITIONS AND PRELIMINARIES}

We denote the set of the real numbers by $\mathbf{R}$ and the $n$-dimensional real space by $\mathbf{R}^{n}$.

Throughout this paper, we use the following notational convention. Variables that range over real numbers are denoted by characters $x, y, z, x_{1}, y_{1}, z_{1}, x_{2}, y_{2}, z_{2}$, .... When there is the need to distinguish between real variables that indicate spatial coordinates and time coordinates, we use $x, y, z, x_{1}, y_{1}, z_{1}, x_{2}, y_{2}, z_{2}, \ldots$ for the former and use $t, t_{1}, t_{2}, \ldots$ for the latter. Variables that range over vectors in $\mathbf{R}^{n}$ and that represent spatial information are denoted by bold characters $\mathbf{x}, \mathbf{x}_{\mathbf{1}}$, $\mathbf{x}_{2}, \ldots$ Real constants are represented by characters $a, b, c, a_{1}, b_{1}, c_{1}, a_{2}, b_{2}, c_{2}, \ldots$. When there is the need to distinguish between real constants that indicate spatial coordinates and time coordinates, we use $a, b, c, a_{1}, b_{1}, c_{1}, a_{2}, b_{2}, c_{2} \ldots$ for the former and use Greek characters $\tau, \tau_{1}, \tau_{2}, \ldots$ for the latter. Finally, bold characters $\mathbf{a}, \mathbf{a}_{1}, \mathbf{a}_{2}, \ldots$ represent constant $n$-dimensional spatial vectors.

Vectors $(\mathbf{a}, \tau)$ containing mixed spatial and temporal information are denoted $p, q, r, p_{1}, q_{1}, r_{1}, p_{2}, q_{2}, r_{2} \ldots$ and variable vectors $(\mathbf{x}, t)$ are represented by characters $u, v, w, u_{1}, v_{1}, w_{1}, u_{2}, v_{2}, w_{2}, \ldots$

\subsection{Semi-algebraic and spatio-temporal databases}

We consider $n$-dimensional spatial figures that move or change over time. A moving figure in $\mathbf{R}^{n}$ can be described by means of an infinite set of tuples $\left(a_{1}, a_{2}, \ldots, a_{n}\right.$, $\tau)$ in $\mathbf{R}^{n} \times \mathbf{R}$, where $\left(a_{1}, a_{2}, \ldots, a_{n}\right)$ represent the $n$-dimensional spatial coordinates of $\left(a_{1}, a_{2}, \ldots, a_{n}, \tau\right)$ and $\tau$ its time coordinate. Obviously, this infinite information needs to be represented finitely in order to be stored in the memory of a computer. In this section, we describe two approaches to model such changing figures, namely the semi-algebraic database model and the spatio-temporal database model. Semialgebraic databases are based on real numbers, while spatio-temporal databases are based on $(n+1)$-dimensional points. Both models resort under the constraint database model [Paredaens et al. 2000; Revesz 2002].

Definition 2.1. A semi-algebraic relation in $\mathbf{R}^{n}$ is a subset of $\mathbf{R}^{n}$ that can be described as a Boolean combination of sets of the form

$$
\left\{\left(x_{1}, x_{2}, \ldots, x_{n}\right) \in \mathbf{R}^{n} \mid p\left(x_{1}, x_{2}, \ldots, x_{n}\right)>0\right\},
$$

with $p$ a polynomial with integer coefficients in the real variables $x_{1}, x_{2}, \ldots, x_{n}$.

In mathematical terms, semi-algebraic relations are known as semi-algebraic sets [Bochnak et al. 1987]. In this paper, we will be mainly interested in semialgebraic relations in real spaces of the form $\mathbf{R}^{(n+1) \times k}$. These relations can be 
viewed as $k$-ary relations over $\mathbf{R}^{n} \times \mathbf{R}$ (i.e., the $n$-dimensional space extended with a time dimension). The next example illustrates this for $k=1$ and $n=2$.

ExAmple 2.1. Figure 1 gives an example of a semi-algebraic relation in $\mathbf{R}^{3}$. This set can be described as follows: $\left\{(x, y, t) \in \mathbf{R}^{2} \times \mathbf{R} \mid x^{2}+y^{2}+t^{2} \leq 1 \vee\left(x^{2}+\right.\right.$ $\left.\left.y^{2}+(t-2)^{2}=1 \wedge t \leq 5 / 2\right) \vee\left(x^{2}+y^{2}+(t-3)^{2}=1 \wedge t>5 / 2\right)\right\}$.

We call a semi-algebraic relation in $\mathbf{R}^{n}$ also a semi-algebraic relation of arity $n$. A semi-algebraic database is essentially a finite collection of semi-algebraic relations. We define this now.

Definition 2.2. A (semi-algebraic) database schema $\sigma$ is a finite set of relation names, where each relation name $R$ has a natural number $\operatorname{ar}(R)$, called its arity, associated to it.

Let $\sigma$ be a database schema. A semi-algebraic database over $\sigma$ is a structure $\mathcal{D}$ over $\sigma$ with domain $\mathbf{R}$ such that, for each relation name $R$ of $\sigma$, the associated relation $R^{\mathcal{D}}$ in $\mathcal{D}$ is a semi-algebraic relation of arity $\operatorname{ar}(R)$.

ExAmple 2.2. Let $\sigma=\{R, S\}$, with $\operatorname{ar}(R)=2$ and $\operatorname{ar}(S)=1$ be a semialgebraic database schema. Then the structure $\mathcal{D}$ given by

$$
\left(\mathbf{R}, R^{\mathcal{D}}=\left\{(x, y) \in \mathbf{R}^{2} \mid x^{2}+y^{2}<1\right\}, S^{\mathcal{D}}=\{x \in \mathbf{R} \mid 0 \leq x \leq 1\}\right)
$$

is an example of a semi-algebraic database over $\sigma$ that contains the open unit disk and the closed unit interval.

We now define spatio-temporal databases. In contrast to semi-algebraic databases, in which points are described by their real coordinates, spatio-temporal databases are based on $(n+1)$-dimensional points. The domain of a spatio-temporal database is $\mathbf{R}^{n} \times \mathbf{R}$. We prefer the notation $\mathbf{R}^{n} \times \mathbf{R}$ over $\mathbf{R}^{n+1}$ for the domain because it stresses the distinction between the time coordinate and the $n$ spatial coordinates of the $(n+1)$-dimensional points.

In the following definition, we work with $\mathbf{R}^{n} \times \mathbf{R}$ as the domain of the spatiotemporal databases and we assume that this underlying dimension $n$ is fixed on before hand. In this paper, we assume, for technical reasons that will become clear in Section 4, that $n \geq 2$.

Throughout this paper we will often use the canonical bijection

$$
\text { can }:\left(\mathbf{R}^{n} \times \mathbf{R}\right)^{k} \rightarrow \mathbf{R}^{(n+1) \times k}
$$

that maps a tuple $\left(\left(\mathbf{a}_{\mathbf{1}}, \tau_{1}\right), \ldots,\left(\mathbf{a}_{\mathbf{k}}, \tau_{k}\right)\right)$ to $\left(a_{1,1}, \ldots, a_{1, n}, \tau_{1}, \ldots, a_{k, 1}, \ldots, a_{k, n}, \tau_{k}\right)$, where for $1 \leq i \leq k$ and $1 \leq j \leq n, a_{i, j}$ denotes the $j$ th real coordinate of the vector $\mathbf{a}_{\mathbf{i}}$.

DeFinition 2.3. A (spatio-temporal) database schema $\sigma$ is a finite set of relation names, where each relation name $R$ has a natural number $\operatorname{ar}(R)$, called its arity, associated to it.

A subset of $\left(\mathbf{R}^{n} \times \mathbf{R}\right)^{k}$ is a spatio-temporal relation of arity $k$ if its image under the canonical bijection can $:\left(\mathbf{R}^{n} \times \mathbf{R}\right)^{k} \rightarrow \mathbf{R}^{(n+1) \times k}$ is a semi-algebraic relation of arity $(n+1) \times k$. 
Let $\sigma$ be a spatio-temporal schema. A spatio-temporal database over $\sigma$ is a structure $\mathbb{\$}$ over $\sigma$ with domain $\mathbf{R}^{n} \times \mathbf{R}$ such that to each relation name $R$ in $\sigma$, a spatio-temporal relation $R^{\mathbb{S}}$ of arity $\operatorname{ar}(R)$ is associated to it.

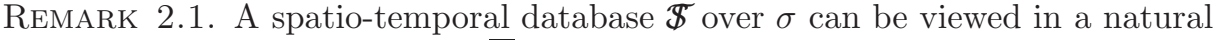
way as a semi-algebraic database $\overline{\mathbb{S}}$ over the semi-algebraic schema $\bar{\sigma}$, which has for each relation name $R$ of $\sigma$, a relation name $\bar{R}$ of arity $(n+1) \times \operatorname{ar}(R)$. For each relation name $R, \bar{R}^{\bar{\Phi}}$ is obtained from $R^{\Phi}$ by applying the canonical bijection can $:\left(\mathbf{R}^{n} \times \mathbf{R}\right)^{\operatorname{ar}(R)} \rightarrow \mathbf{R}^{(n+1) \times a r(R)}$. We will use the notation introduced here, throughout the paper.

Following this remark, we observe that spatio-temporal relations and databases can be finitely encoded and stored by means of the systems of polynomial equalities and inequalities (i.e., by means of a quantifier-free formula of first-order logic over the reals with,$+ \times,<$ and the constants 0 and 1 ) that describe the associated semi-algebraic relations and databases.

REMARK 2.2. Throughout this paper, we assume that databases are finitely encoded by systems of polynomial equations and that a specific data structure is fixed (possible data structures are dense or sparse representations of polynomials). The specific choice of data structure is not relevant to the topic of this paper, but we assume that one is fixed. When we talk about computable queries later on, we mean Turing computable with respect to the chosen encoding and data structures.

The model presented here and the results in this paper can be extended straightforwardly to the situation where spatio-temporal relations are accompanied by classical thematic information, like the typical alpha-numeric data you find in business applications and also, in combination with spatial data, in Geographical Information Systems. However, because the problem that is discussed here is captured by this simplified model, we stick to it for reasons of simplicity of exposition.

EXAMPLE 2.3. Figure 1 in the Introduction gives an illustration of a spatiotemporal database over a schema $\sigma=\{R\}$ with underlying dimension 2, where $R$ has arity 1 . It shows at its beginning, i.e., at $t=-1$, a single point in the origin of $\mathbf{R}^{2}$. Then it shows a disk whose radius increases and later decreases and ends in a point at moment $t=1$, followed by a circle whose radius increases, decreases, increases and then shrinks to a point.

Definition 2.4. Let $\sigma$ be a spatio-temporal schema and let $\$$ be a spatiotemporal database over $\sigma$ with underlying dimension $n$. Let $R$ be a relation name in $\sigma$ and let $\tau_{0}$ be a real number representing a moment in time. We call the subset

$$
R^{\Phi} \cap\left(\mathbf{R}^{n} \times\left\{\tau_{0}\right\}\right)^{\operatorname{ar}(R)}
$$

of $\left(\mathbf{R}^{n} \times\left\{\tau_{0}\right\}\right)^{\operatorname{ar}(R)}$ the snapshot of $R$ at the moment $\tau_{0}$. The snapshot of the spatio-temporal database $\mathbb{S}$ at the moment $\tau_{0}$ is the finite set of snapshots of all its relations at $\tau_{0}$.

EXAmPle 2.4. For the spatio-temporal relation depicted in Figure 1, the snapshot at -1 is $\{(0,0,-1)\}$, the snapshot at 0 is the closed unit disk in the plane $t=0$ and the snapshot at 5 is the empty set.

ACM Journal Name, Vol. V, No. N, 20YY. 


\subsection{Spatio-temporal and semi-algebraic database queries}

Here, we define spatio-temporal and semi-algebraic database queries as computable mappings of some type. In the next section, we will argue that not all such mappings should be regarded as "reasonable" queries and that further conditions on the nature of these mappings have to be imposed.

Definition 2.5. Let $\sigma$ be a semi-algebraic database schema. A $k$-ary semialgebraic database query $Q$ over $\sigma$ is a partial, computable mapping (in the sense of Remark 2.2) from the set of semi-algebraic databases over $\sigma$ to the set of $k$-ary semi-algebraic relations.

Definition 2.6. Let $\sigma$ be a spatio-temporal database schema and let us consider input spatio-temporal databases over $\sigma$ with underlying dimension $n$. A $k$-ary $n$-dimensional spatio-temporal database query $Q$ over $\sigma$ is a partial, computable mapping (in the sense of Remark 2.2) from the set of spatio-temporal databases over $\sigma$ to the set of $k$-ary spatio-temporal relations with underlying dimension $n$.

We also call a $k$-ary $n$-dimensional spatio-temporal database query a spatio-temporal database query of output type $(n, k)$.

Note that we restrict spatio-temporal database queries to preserve the underlying dimension of the input database.

EXAmPle 2.5. Let $\sigma=\{R\}$, where $R$ has arity 1 and let the underlying dimension be 2 . The query that selects those snapshots from the relation $R$ where $R$ shows a circle is a spatio-temporal database query of output type $(2,1)$. Applied to the database of Example 2.3 and shown in Figure 1, this query returns the union of its snapshots in the open time interval $] 1,4[$.

There is a natural way to see spatio-temporal queries as semi-algebraic queries, that is captured in the following definition of equivalence of queries.

Definition 2.7. Let $\sigma$ be a spatio-temporal database schema and let us consider input spatio-temporal databases over $\sigma$ with underlying dimension $n$. Let $\bar{\sigma}$ be the corresponding semi-algebraic database schema (see Remark 2.1). Let $Q$ be a $k$-ary $n$-dimensional spatio-temporal database query over $\sigma$ and let $\bar{Q}$ be a $((n+1) \times k)$ ary semi-algebaric database query over $\bar{\sigma}$. We say that $Q$ and $\bar{Q}$ are equivalent if

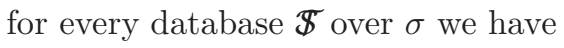

$$
\overline{Q(\bar{\Phi})}=\bar{Q}(\bar{\Phi})
$$

\subsection{First-order logic and its extension with a while loop as a spatio-temporal query} language

First-order logic over the field of the real numbers, $\mathrm{FO}(+, \times,<, 0,1)$ for short, has been well-studied as a query language for spatial databases [Kanellakis et al. 1995; Paredaens et al. 1994; Paredaens et al. 2000]. In the setting of spatio-temporal databases, it can be used as a query language in a similar way. We introduce 
$\mathrm{FO}(+, \times,<, 0,1)$ here as a spatio-temporal query language, beit on semi-algebraic databases that represent spatio-temporal databases.

Definition 2.8. Let $\sigma=\left\{R_{1}, R_{2}, \ldots, R_{m}\right\}$ be a spatio-temporal database schema and let us consider queries working on input databases over $\sigma$ with underlying dimension $n$. Let $\bar{R}_{i} \quad(1 \leq i \leq m)$ be the corresponding semi-algebraic relation names of arity $(n+1) \times \operatorname{ar}\left(R_{i}\right)$ (we follow the notation of Remark 2.1) and let $\bar{\sigma}$ be the semi-algebraic schema $\left\{\bar{R}_{1}, \bar{R}_{2}, \ldots, \bar{R}_{m}\right\}$.

Let $\varphi\left(\mathbf{x}_{\mathbf{1}}, t_{1}, \mathbf{x}_{\mathbf{2}}, t_{2}, \ldots, \mathbf{x}_{\mathbf{k}}, t_{k}\right)$, be a first-order logic formula over the alphabet $\left(+, \times, 0,1,<, \bar{R}_{1}, \bar{R}_{2}, \ldots, \bar{R}_{m}\right)$. If $\mathbf{x}_{\mathbf{i}}=\left(x_{i, 1}, \ldots, x_{i, n}\right)$, then the free variables of $\varphi$ are $x_{1,1}, \ldots, x_{1, n}, t_{1}, x_{2,1}, \ldots, x_{2, n}, t_{2}, \ldots, x_{k, 1}, \ldots, x_{k, n}, t_{k}$. The formula $\varphi$ expresses a semi-algebraic $((n+1) \times k)$-ary query $\bar{Q}$ which is equivalent to a $k$-ary $n$-dimensional spatio-temporal query $Q$. For each input spatio-temporal database $\$$ over $\sigma, Q(\$)$ is defined as the set of points $\left(\left(\mathbf{a}_{\mathbf{1}}, \tau_{1}\right),\left(\mathbf{a}_{\mathbf{2}}, \tau_{2}\right), \ldots,\left(\mathbf{a}_{\mathbf{k}}, \tau_{k}\right)\right)$ of $\left(\mathbf{R}^{n} \times \mathbf{R}\right)^{k}$ such that

$$
\left(\mathbf{R},+, \times, 0,1,<, \bar{R}_{1}^{\bar{\Phi}}, \bar{R}_{2}^{\bar{\Phi}}, \ldots, \bar{R}_{m}^{\bar{\Phi}}\right) \models \varphi\left[\mathbf{a}_{1}, \tau_{1}, \mathbf{a}_{2}, \tau_{2}, \ldots, \mathbf{a}_{\mathbf{k}}, \tau_{k}\right],
$$

where $\varphi\left[\mathbf{a}_{\mathbf{1}}, \tau_{1}, \mathbf{a}_{\mathbf{2}}, \tau_{2}, \ldots, \mathbf{a}_{\mathbf{k}}, \tau_{k}\right]$ denotes the formula $\varphi\left(\mathbf{x}_{\mathbf{1}}, t_{1}, \mathbf{x}_{\mathbf{2}}, t_{2}, \ldots, \mathbf{x}_{\mathbf{k}}, t_{k}\right)$ with its free variables instantiated by $\mathbf{a}_{\mathbf{1}}, \tau_{1}, \mathbf{a}_{\mathbf{2}}, \tau_{2}, \ldots, \mathbf{a}_{\mathbf{k}}, \tau_{k}$.

We will refer to the first-order query language, introduced here, as $\mathrm{FO}(+, \times,<$, $\left.0,1, \bar{R}_{1}, \bar{R}_{2}, \ldots, \bar{R}_{m}\right)$, or, if the schema is clear from the context, as $\mathrm{FO}(+, \times,<, 0$, $1)$.

Example 2.6. As in Example 2.5, let $\sigma=\{R\}$, where $R$ has arity 1 and let the underlying dimension be 2 . The formula $\left(\exists x_{0}\right)\left(\exists y_{0}\right)(\exists r>0)\left(\left(x-x_{0}\right)^{2}+(y-\right.$ $\left.\left.y_{0}\right)^{2}=r^{2} \Leftrightarrow \bar{R}(x, y, t)\right)$ expresses a spatio-temporal query of output type $(2,1)$. It selects those snapshots from a spatio-temporal relation $R$ where $R$ shows a circle. As mentioned, applied to the database of Example 2.3, this query returns all its snapshots in the time interval $] 1,4[$.

We remark that the formalism of semi-algebraic and spatio-temporal databases and the first-order query language introduced here, fits within the framework of constraint databases [Paredaens et al. 2000; Revesz 2002]. It is well known that $\mathrm{FO}(+, \times,<, 0,1)$-formulas can be effectively evaluated in the constraint model and therefore also in this context. It is also known that the output can be represented in the same constraint formalism [Paredaens et al. 2000; Van den Bussche 2000], i.e. by a quantifier-free formula over $(+, \times, 0,1,<)$.

REMARK 2.3. An arbitrary FO-formula does not necessarily express a spatiotemporal database query as shown by the following example. The formula

$$
(\exists t) \bar{R}\left(x_{1}, x_{2}, t\right)
$$

expresses the projection of the spatio-temporal relation $R$ on the spatial $\left(x_{1}, x_{2}\right)$ plane. The formula returns a set of couples $\left(x_{1}, x_{2}\right)$ in $\mathbf{R}^{2}$ that form a semi-algebraic set with a purely spatial meaning.

We end this section by specifying the programming language $\mathrm{FO}(+, \times,<, 0,1$, $\bar{\sigma})+$ While which is known to be computationally complete on semi-algebraic databases [Gyssens et al. 1999]. Essentially, this language is an extension of $\mathrm{FO}(+$, 
$\times,<, 0,1, \bar{\sigma})$ with assignments and a While loop. The use of similar languages will be illustrated in Section 5. We also refer to [Gyssens et al. 1999; Van den Bussche 2000] for illustrations.

Definition 2.9. Let $\sigma$ be a spatio-temporal database schema. Syntactically, a program in the language $\mathrm{FO}(+, \times,<, 0,1, \bar{\sigma})+$ While is a finite sequence of statements and while-loops. It is assumed there is a sufficient supply of new relation variables, each with an appropriate arity.

(i) Each statement has the form

$$
\bar{R}:=\left\{\left(x_{1}, \ldots, x_{k}\right) \mid \varphi\left(x_{1}, \ldots, x_{k}\right)\right\} ; .
$$

Here, $\bar{R}$ is a new relation variable with assigned arity $k$ (the variables $x_{i}$ range over $\mathbf{R}$ ) and $\varphi$ is a formula in $\mathrm{FO}\left(+, \times,<, 0,1, \overline{\sigma^{\prime}}\right)$, where $\overline{\sigma^{\prime}}$ is the set of relation names containing the elements of $\bar{\sigma}$ together with the relation variables introduced in previous statements of the program.

(ii) A while-loop has the form

$$
\text { while } \varphi \text { do } P \text {; }
$$

where $P$ is a program and $\varphi$ is a sentence in $\mathrm{FO}\left(+, \times,<, 0,1, \overline{\sigma^{\prime}}\right)$, where $\overline{\sigma^{\prime}}$ is again the set of relation names containing the elements of $\bar{\sigma}$ together with the relation variables introduced in previous statements of the program.

(iii) One of the relation names occurring in the program is designated as the output relation and is named $\bar{R}_{\text {out }}$.

Semantically, a program in the query language $\mathrm{FO}(+, \times,<, 0,1, \bar{\sigma})+$ While expresses a spatio-temporal query as soon as $\bar{R}_{\text {out }}$ is assigned a return value. The execution of an $\mathrm{FO}(+, \times,<, 0,1, \bar{\sigma})+$ While-program applied to an input database is performed step-by-step. A statement is executed by first evaluating the $\mathrm{FO}(+, \times$, $<, 0,1, \bar{\sigma})$-formula on the right hand side on the input database together with the new relations resulting from previous statements. Next, the result of the evaluation of the right hand side is assigned to the relation variable on the left-hand side. The effect of a while loop is to execute the body as long as the condition $\varphi$ evaluates to true.

Note that these programs are not guaranteed to halt. For those input databases it does not, the query represented by the program is not defined on that particular input database.

\section{SPATIO-TEMPORAL GENERICITY}

As stated in the introduction, we are interested in spatio-temporal database queries that are invariant under the elements of a certain spatio-temporal transformation group (for function composition)

$$
\mathcal{F}=\left\{f \mid f=\left(f_{1}, f_{2}, \ldots, f_{n}, f_{t}\right): \mathbf{R}^{n} \times \mathbf{R} \rightarrow \mathbf{R}^{n} \times \mathbf{R}\right\} .
$$

The idea is that the result of spatio-temporal queries should be largely independent of the particular coordinate system in which the data are presented. In this section, we formalize this idea by the notion of $\mathcal{F}$-genericity. 
In the remainder of this section, we look at different types of transformation groups and we impose two further conditions on these transformations. Firstly, we look at purely temporal conditions. Secondly, we look at purely spatial or spatiotemporal conditions that reflect the nature of the queries one is interested in. We also look at transformation groups that are suited for applications in which physical notions such as velocity and acceleration are of importance.

\subsection{Definition of spatio-temporal genericity}

Let $f: \mathbf{R}^{n} \times \mathbf{R} \rightarrow \mathbf{R}^{n} \times \mathbf{R}$ be a function, let $R$ be a spatio-temporal relation name of arity $k$ and let $R^{\mathbb{S}}$ be a relation instance with underlying dimension $n$. In the following, we use the notation $f\left(R^{\mathbb{\Phi}}\right)$ to abbreviate the set $\left\{\left(f\left(\mathbf{a}_{1}, \tau_{1}\right), f\left(\mathbf{a}_{2}, \tau_{2}\right)\right.\right.$, $\left.\left.\ldots, f\left(\mathbf{a}_{k}, \tau_{k}\right)\right) \in\left(\mathbf{R}^{n} \times \mathbf{R}\right)^{k} \mid\left(\mathbf{a}_{1}, \tau_{1}, \mathbf{a}_{2}, \tau_{2}, \ldots, \mathbf{a}_{k}, \tau_{k}\right) \in R^{\mathscr{\Phi}}\right\}$.

DeFinition 3.1. Let $Q$ be a spatio-temporal database query that takes databases of signature $\sigma=\left\{R_{1}, \ldots, R_{m}\right\}$ with underlying dimension $n$ as input. Let $\mathcal{F}=\left\{f \mid f: \mathbf{R}^{n} \times \mathbf{R} \rightarrow \mathbf{R}^{n} \times \mathbf{R}\right\}$ be a spatio-temporal transformation group. We say that $Q$ is $\mathcal{F}$-generic if, for any $f$ in $\mathcal{F}$ and for each pair of spatio-temporal databases $\mathbb{\$}_{1}$ and $\mathbb{S}_{2}$ over $\sigma$, the fact that $\mathbb{S}_{2}=\left(R_{1}^{\mathbb{S}_{2}}, \ldots, R_{m}^{\Phi_{2}}\right)=\left(f\left(R_{1}^{\mathbb{S}_{1}}\right), \ldots, f\left(R_{m}^{\mathbb{S}_{1}}\right)\right)$ implies that $f\left(Q\left(\mathbb{S}_{1}\right)\right)=Q\left(\mathbb{S}_{2}\right)$.

This definition will be illustrated in Section 3.5.

It is clear that if a query is $\mathcal{F}$-generic, it is also $\mathcal{F}^{\prime}$-generic for any subgroup $\mathcal{F}^{\prime}$ of $\mathcal{F}$.

\subsection{Temporal restrictions on the transformations}

It is very natural to describe spatio-temporal events with the notions "before", "after" and "co-temporal". For instance, when two people arrive shortly after each other, we say "Mary arrived before Jane" rather than "Mary arrived at 9:31 and Jane at 9:35". Another example is any kind of race. The winner is the one that finishes first. So, foremost the order of arrival of the participants matters. Exact time moments are only important in very specific situations.

We start with the definition of a spatio-temporal event.

Definition 3.2. An event is a subset of $\mathbf{R}^{n} \times \mathbf{R}$. The projection of an event $A$ on the time-axis is denoted by $\pi_{t}(A)$ and called the time-domain of $A$.

Let $A$ and $B$ be events. In the terminology of Allen's interval calculus [Allen 1983; Allen and Ferguson 1994], $A$ and $B$ are called co-temporal if $\pi_{t}(A)=\pi_{t}(B)$ (we denote this by $A={ }_{t} B$ ). Allen says $A$ is before $B$ if $t_{A}<t_{B}$ for all $t_{A} \in \pi_{t}(A)$ and all $t_{B} \in \pi_{t}(B)$ (we denote this by $A<_{t} B$ ).

Remark that $A \leq_{t} B:=\left(A={ }_{t} B\right.$ or $\left.A<_{t} B\right)$ is a pre-order on events.

Definition 3.3. We say that a transformation $f: \mathbf{R}^{n} \times \mathbf{R} \rightarrow \mathbf{R}^{n} \times \mathbf{R}$ preserves the order of events if for all events $A$ and $B, A={ }_{t} B$ implies $f(A)={ }_{t} f(B)$ and $A<_{t} B$ implies $f(A)<_{t} f(B)$.

Proposition 3.1. A transformation $f=\left(f_{1}, f_{2}, \ldots, f_{n}, f_{t}\right): \mathbf{R}^{n} \times \mathbf{R} \rightarrow \mathbf{R}^{n} \times$ $\mathbf{R}:(\mathbf{x}, t) \mapsto\left(f_{1}(\mathbf{x}, t), \ldots, f_{n}(\mathbf{x}, t), f_{t}(\mathbf{x}, t)\right)$ preserves the order of events if and only if $f_{t}$ is a strictly monotone increasing bijection of $t$ alone.

ACM Journal Name, Vol. V, No. N, $20 Y Y$. 
Proof. The if-direction is straightforward. To prove the other direction, let $f=\left(f_{1}, f_{2}, \ldots, f_{n}, f_{t}\right)$ be a transformation of $\mathbf{R}^{n} \times \mathbf{R}$. Consider any two events $A=\left\{\left(a_{1}, a_{2}, \ldots, a_{n}, \tau\right)\right\}$ and $B=\left\{\left(a_{1}^{\prime}, a_{2}^{\prime}, \ldots, a_{n}^{\prime}, \tau\right)\right\}$. Since $A={ }_{t} B$, then $f_{t}\left(a_{1}, a_{2}, \ldots, a_{n}, \tau\right)=f_{t}\left(a_{1}^{\prime}, a_{2}^{\prime}, \ldots, a_{n}^{\prime}, \tau\right)$. This shows that $f_{t}$ is a function of $t$ alone.

Consider any two events $A=\left\{\left(a_{1}, a_{2}, \ldots, a_{n}, \tau_{1}\right)\right\}$ and $B=\left\{\left(a_{1}, a_{2}, \ldots, a_{n}, \tau_{2}\right)\right\}$ with $\tau_{1}<\tau_{2}$. Since $A<_{t} B$, then $f_{t}\left(\tau_{A}\right)<f_{t}\left(\tau_{B}\right)$. This shows that $f_{t}$ is a strictly monotone function of $t$.

The transformation groups that we consider are all groups with respect to the composition operator $\circ$ of functions. Therefore, for every transformation $f$ also its inverse exists, and hence $f$ is a bijection. Given the fact that the component $f_{t}$ is a function of $t$ alone, it has to be a bijection too.

We require that transformations preserve the order of events. We can therefore write the transformation groups of interest as a product of groups, i.e., $\mathcal{F}=\left(\mathcal{F}_{s t}, \mathcal{F}_{t}\right)$, where

$$
\left(\mathcal{F}_{s t}, \mathcal{F}_{t}\right)=\left\{\left(f_{s t}, f_{t}\right) \mid f_{s t}=\left(f_{1}, f_{2}, \ldots, f_{n}\right): \mathbf{R}^{n} \times \mathbf{R} \rightarrow \mathbf{R}^{n} \text { and } f_{t}: \mathbf{R} \rightarrow \mathbf{R}\right\} .
$$

The particular groups $\mathcal{F}_{t}$ that we will consider in this paper are:

$-\mathcal{A}_{t}=\{t \mapsto a t+b \mid a, b \in \mathbf{R}$ and $a>0\}$, i.e., the monotone affinities of the time-line;

$-\mathcal{T}_{t}=\{t \mapsto t+b \mid b \in \mathbf{R}\}$, i.e., the translations of the time-line; and

$-I d_{t}=\{\mathrm{id}\}$, i.e., the identity of time.

Invariance with respect to this type of transformations of time is often encountered in physics [Desloge 1982].

\subsection{Spatial and spatio-temporal restrictions on transformations}

In the following, we consider transformations coming from practical situations where moving objects are monitored from a fixed position or situations where a fixed object is observed from a moving position. The frame of reference is therefore changing in a time-dependent way. In real life, this continuous change of reference system arises in different kinds of situations. For example, when a moving person is watching an event, his/her description of that event will be related to his/her position and orientation at each time moment. When this person moves along a straight line at constant speed, the transformation that describes this continuous change of reference system would be a time-dependent affinity.

In this paper, we only look at transformations that have an algebraic description. The general form of the transformation groups $\mathcal{F}_{s t}$ that we consider have elements of the form:

$$
\left(\begin{array}{c}
x_{1} \\
x_{2} \\
\vdots \\
x_{n} \\
t
\end{array}\right) \mapsto\left(\begin{array}{cccc}
\alpha_{11}(t) & \alpha_{12}(t) & \cdots & \alpha_{1 n}(t) \\
\alpha_{21}(t) & \alpha_{22}(t) & \cdots & \alpha_{2 n}(t) \\
\vdots & \vdots & \cdots & \vdots \\
\alpha_{n 1}(t) & \alpha_{n 2}(t) & \cdots & \alpha_{n n}(t)
\end{array}\right) \cdot\left(\begin{array}{c}
x_{1} \\
x_{2} \\
\vdots \\
x_{n}
\end{array}\right)+\left(\begin{array}{c}
\beta_{1}(t) \\
\beta_{2}(t) \\
\vdots \\
\beta_{n}(t)
\end{array}\right)
$$


where the $\alpha_{i j}$ and $\beta_{i}$ are functions from $\mathbf{R}$ to $\mathbf{R}$. Furthermore, we require that the transformation groups that we consider are "semi-algebraic" (we give a precise definition in Section 5.3).

We will consider the following groups $\mathcal{F}_{s t}$ of transformations:

$-\mathcal{A}_{s t}$ is the group of transformations of the above form where the $\alpha_{i j}(t)$ and $\beta_{i}(t)$ are arbitrary functions of $t$ such that the matrix of the $\alpha_{i j}(t)$ has an inverse for each value of $t$, i.e., these are the time-dependent affinities;

$-\mathcal{A}_{s t}^{f}$ is the subgroup of $\mathcal{A}_{s t}$ consisting of transformations for which the functions $\alpha_{i j}(t)$ and $\beta_{i}(t)$ only take a finite number of values, i.e., functions that are piecewise constant;

$-\mathcal{A}_{s t}^{c}$ is the subgroup of $\mathcal{A}_{s t}^{f}$ consisting of transformations for which the functions $\alpha_{i j}(t)$ are constants and $\beta_{i}(t)$ are linear functions of $t$;

$\mathcal{S}_{s t}, \mathcal{S}_{s t}^{f}$ and $\mathcal{S}_{s t}^{c}$ are subgroups of $\mathcal{A}_{s t}, \mathcal{A}_{s t}^{f}$ and $\mathcal{A}_{s t}^{c}$ respectively, where the matrix of the $\alpha_{i j}(t)$ represents at each moment a similarity, i.e. the composition of an isometry (given by a matrix with determinant 1 ) and a scaling (given by a nonzero multiple of the unit matrix);

$\mathcal{I}_{s t}, \mathcal{I}_{s t}^{f}, \mathcal{I}_{s t}^{c}$ are the subgroups of the above groups where the determinant of the matrix consisting of the $\alpha_{i j}(t)$ equals 1 at each moment, i.e., this matrix determines an isometry;

$-\mathcal{T}_{s t}, \mathcal{T}_{s t}^{f}, \mathcal{T}_{s t}^{c}$ are the subgroups of the above groups where the matrix consisting of the $\alpha_{i j}(t)$ is the identity matrix, i.e., these are groups of translations.

\subsection{Physical transformation groups}

The following groups are of interest when notions such as velocity, acceleration and force are important in an application. These transformation groups can be found by solving the differential equations that express that these physical entities are preserved [Desloge 1982]. We consider these notions for arbitrary and rigid motions, respectively. A rigid motion is a motion that preserve the shape of a moving body or moving figure, i.e., it is an isometric movement. To study the velocity and acceleration of a moving body, we only consider the movement of the center of mass of that figure and do not take into account the changes in shape of the body.

The transformation groups of interest here are the following.

$-\mathcal{V}_{s t}$ is the subgroup of $\mathcal{A}_{s t}^{c}$ where the $\beta_{i}$ are constants. This group of transformations preserves the velocity vector of a moving figure.

$-\mathcal{V}(\mathcal{R})_{s t}$ is the subgroup of $\mathcal{I}_{s t}^{c}$ where the $\beta_{i}$ are constants. This group of transformations preserves the velocity vector of a moving figure in rigid motion.

$-\mathcal{A C}_{s t}$ is the group $\mathcal{A}_{s t}^{c}$. This group of transformations preserves the acceleration vector of a moving object.

$-\mathcal{A C}(\mathcal{R})_{s t}$ is the group $\mathcal{I}_{s t}^{c}$. This group of transformations preserves the acceleration vector of a moving figure in rigid motion.

In physics it is customary to consider only translations for what concerns the time dimension, i.e., the transformations in the group $\mathcal{T}_{t}$. The group $\left(\mathcal{A C}(\mathcal{R})_{s t}, \mathcal{T}_{t}\right)$ 
is also known as the group of the Galilei transformations [Desloge 1982]. It is particularly useful because all laws of classical mechanics are invariant for this group of transformations of space-time [Desloge 1982].

\subsection{Examples of generic queries}

We end this section with a number of examples of queries that are generic for some of the genericity classes that we have introduced above.

Suppose in some city, an experiment is set up to evaluate the traffic situation. A number of probe cars (for simplicity, we assume two) is continuously driving around the city in a random way. The trajectories of the cars are stored in a spatio-temporal database, of underlying dimension 2, with schema $\sigma=\{\operatorname{car} A, \operatorname{car} B\}$, where the relations $\operatorname{car} A$ and $\operatorname{car} B$ both have arity 1 .

In these examples, we assume that time is measured in seconds and distance is measured in meters.

We now give some example queries, and indicate for each the transformation groups it is generic for.

EXAMPLE 3.1. $Q_{1}$ : Does the route followed by car A self-intersect more often than the route followed by car $B$ does?

This query is $\left(\mathcal{V}_{s t}, \mathcal{A}_{t}\right)$-generic, but not $\left(\mathcal{A}_{s t}, \mathcal{A}_{t}\right)$-generic, for instance. It is not expressible in first-order logic. In Section 5, we will give a "program" expressing this query.

EXAMPle 3.2. $Q_{2}$ : Give the places and time moments where it is true for car $A$ that when it reaches them, it is standing still at that spot for at least 300 more seconds, (i.e., where and when did car A encounter a traffic jam?).

This query is $\left(\mathcal{V}_{s t}, \mathcal{T}_{t}\right)$-generic. Indeed, the fact that a car has speed zero (when it is standing still) has to be preserved, which requires the group $\mathcal{V}_{s t}$, and the length of time intervals has to be preserved, which requires $\mathcal{T}_{t}$.

This query is expressed by the following $\mathrm{FO}(+, \times,<, 0,1, \overline{\operatorname{car} A})$-formula:

$$
\varphi_{2}(x, y, t):=\left(\overline{\operatorname{car} A}(x, y, t) \wedge\left(\forall t_{2}\right)\left(\left(t \leq t_{2} \wedge t_{2} \leq t+300\right) \rightarrow \overline{\operatorname{carA}}\left(x, y, t_{2}\right)\right) .\right.
$$

EXAmple 3.3. $Q_{3}:$ Was there a collision between car $A$ and car $B$ ?

This query is $\left(\mathcal{A}_{s t}, \mathcal{A}_{t}\right)$-generic. This query is expressed by the following $\mathrm{FO}(+$, $\times,<, 0,1, \overline{\operatorname{car} A}, \overline{\operatorname{car} B})$-formula:

$$
\varphi_{3}:=(\exists x)(\exists y)(\exists t)(\overline{\operatorname{car} A}(x, y, t) \wedge \overline{\operatorname{car} B}(x, y, t)) .
$$

Example 3.4. $Q_{4}$ : Did car A pass at 500 meters north of car $B$ at time moment $t=5930$ ?

This query is $\left(\mathcal{T}_{s t}, I d_{t}\right)$-generic. This query is expressed by the following $\mathrm{FO}(+$, $\times,<, 0,1, \overline{\operatorname{car} A}, \overline{\operatorname{car} B}$ )-formula:

$$
\varphi_{4}:=\left(\exists x_{1}\right)\left(\exists y_{1}\right)\left(\exists y_{2}\right)\left(\overline{\operatorname{carA}}\left(x_{1}, y_{1}, 5930\right) \wedge \overline{\operatorname{carB}}\left(x_{1}, y_{2}, 5930\right) \wedge y_{1}=y_{2}+500\right) \text {. }
$$


EXAmPle 3.5. $Q_{5}:$ Did car A encounter any "empty roads"? (I.e., were there parts of its trajectory where it could drive at constant speed in a straight line for at least 6000 seconds?)

This query is $\left(\mathcal{A C}_{s t}, \mathcal{T}_{t}\right)$-generic. The fact that a car drives at constant speed (i.e., has an acceleration of zero) has to be preserved. Note that, because the car's movement is a polynomial function of time, driving at constant speed means driving in a straight line. Query $Q_{5}$ can be expressed by the following $\mathrm{FO}(+, \times,<$, $0,1, \overline{\operatorname{car} A})$-formula:

$$
\begin{array}{r}
\varphi_{5}:=\left(\exists t_{1}\right)\left(\exists t_{2}\right)\left(\exists x_{1}\right)\left(\exists y_{1}\right)\left(\exists x_{2}\right)\left(\exists y_{2}\right)\left(\overline{\operatorname{carA}}\left(x_{1}, y_{1}, t_{1}\right) \wedge \overline{\operatorname{carA}}\left(x_{2}, y_{2}, t_{2}\right) \wedge\right. \\
t_{2}=t_{1}+6000 \wedge\left(\forall t_{3}\right)\left(( t _ { 1 } \leq t _ { 3 } \wedge t _ { 3 } \leq t _ { 2 } ) \rightarrow ( \exists x _ { 3 } ) ( \exists y _ { 3 } ) \left(\overline{\operatorname{car} A}\left(x_{3}, y_{3}, t_{3}\right) \wedge\right.\right. \\
\left.\left.\left.\left(t_{2}-t_{1}\right) x_{3}=\left(t_{2}-t_{3}\right) x_{1}+\left(t_{3}-t_{1}\right) x_{2} \wedge\left(t_{2}-t_{1}\right) y_{3}=\left(t_{2}-t_{3}\right) y_{1}+\left(t_{3}-t_{1}\right) y_{2}\right)\right)\right) .
\end{array}
$$

This completes the examples section. We return to these examples later on, when we have defined point languages.

\section{SOUND AND COMPLETE LANGUAGES FOR THE GENERIC FIRST-ORDER SPATIO-TEMPORAL QUERIES}

In this section, we study the $\left(\mathcal{F}_{s t}, \mathcal{F}_{t}\right)$-generic queries that are expressible in $\mathrm{FO}(+$, $\times,<, 0,1)$. To start with, we give a general undecidability result. We prove that it is undecidable whether a query is $\left(\mathcal{F}_{s t}, \mathcal{F}_{t}\right)$-generic, for any nontrivial group $\left(\mathcal{F}_{s t}\right.$, $\left.\mathcal{F}_{t}\right)$.

Next, we show that $\left(\mathcal{F}_{s t}, \mathcal{F}_{t}\right)$-generic $\mathrm{FO}(+, \times,<, 0,1)$-queries are recursive enumerable, however. We do this by syntactically specifying languages that capture the $\left(\mathcal{F}_{s t}, \mathcal{F}_{t}\right)$-generic queries, for all groups $\left(\mathcal{F}_{s t}, \mathcal{F}_{t}\right)$ listed in Section 3.3 and Section 3.4.

The strategy to prove the following Theorem was introduced by Paredaens, Van den Bussche and Van Gucht [Paredaens et al. 1994].

Let $\mathbf{N}$ denote the set of the natural numbers.

Theorem 4.1. For all non-trivial groups $\left(\mathcal{F}_{s t}, \mathcal{F}_{t}\right)$ mentioned in the previous section, $\left(\mathcal{F}_{s t}, \mathcal{F}_{t}\right)$-genericity of spatio-temporal $\mathrm{FO}(+, \times,<, 0,1, \sigma)$-queries is undecidable, where $\sigma$ is a non-empty schema.

Proof. Let $\mathcal{F}$ be a group of transformations of $\mathbf{R}^{n} \times \mathbf{R}$ that contains an element $f_{0}$ that does not map $(0,0)$ to itself. We show that $\mathcal{F}$-genericity of spatio-temporal queries over a certain schema $\sigma=\{R\}$, where $R$ is a one-dimensional unary spatiotemporal relation, of output type $(1,1)$ is undecidable. For other non-empty schemas the proof is similar. We will do this by reducing deciding the truth of sentences of the $\forall *$-fragment of number theory to the genericity question. The $\forall *$-fragment of number theory is known to be undecidable since Hilbert's 10th problem [Matijasevich 1993] can be formulated in it.

We encode a natural number $n$ by the unary one-dimensional spatio-temporal relation

$$
\operatorname{enc}(n):=\{(0,0),(1,0), \ldots,(n, 0)\} .
$$

ACM Journal Name, Vol. V, No. N, 20YY. 
A ( $k$-dimensional) vector of natural numbers $\left(n_{1}, n_{2}, \ldots, n_{k}\right)$ is encoded by the relation

$$
\begin{gathered}
\operatorname{enc}\left(n_{1}, n_{2}, \ldots, n_{k}\right):=\operatorname{enc}\left(n_{1}\right) \cup\left(\operatorname{enc}\left(n_{2}\right)+\left(n_{1}+2,0\right)\right) \cup \\
\ldots \cup\left(\operatorname{enc}\left(n_{k}\right)+\left(n_{1}+2+\cdots+n_{k-1}+2,0\right)\right) .
\end{gathered}
$$

For fixed $k$, the corresponding decoding is expressible in $\mathrm{FO}(+, \times,<, 0,1)$. We thus associate to the first-order sentence $\left(\forall n_{1}\right) \cdots\left(\forall n_{k}\right) \varphi\left(n_{1}, \ldots, n_{k}\right)$ of number theory to the following spatio-temporal query $Q_{\varphi}$ over the input schema $\sigma=\{R\}$ :

if $\bar{R}$ encodes a vector $\left(n_{1}, \ldots, n_{k}\right) \in \mathbf{N}^{k}$

then

if $\varphi\left(n_{1}, \ldots, n_{k}\right)$

then return $\emptyset$

else return $\{(0,0)\}$

else return $\emptyset$.

This query is expressible in $\mathrm{FO}(+, \times,<, 0,1)$.

Claim. The query $Q_{\varphi}$ is $\mathcal{F}$-generic if and only if the sentence $\left(\forall n_{1}\right) \cdots\left(\forall n_{k}\right) \varphi\left(n_{1}\right.$, $\ldots, n_{k}$ ) holds in the natural numbers.

Now, we prove this claim. First, suppose that, for all $\left(n_{1}, \ldots, n_{k}\right) \in \mathbf{N}^{k}, \varphi\left(n_{1}\right.$, $\left.\ldots, n_{k}\right)$ holds. Let $R$ be a one-dimensional unary spatio-temporal relation and let $f$ be some transformation of $\mathcal{F}$. We have to prove that

$$
f\left(Q_{\varphi}(R)\right)=Q_{\varphi}(f(R)) .
$$

The result of $Q_{\varphi}(R)$ will always be $\emptyset$ : either $R$ does not encode a vector $\left(n_{1}, \ldots\right.$, $\left.n_{k}\right)$, or it does and $\varphi\left(n_{1}, \ldots, n_{k}\right)$ holds. For the same reason, $Q_{\varphi}(f(R))$ also equals $\emptyset$. The transformation $f$ maps $\emptyset$ to $\emptyset$, hence $f\left(Q_{\varphi}(R)\right)=\emptyset$, which concludes the first part of the proof.

Now assume that there exists an $\left(n_{1}^{0}, \ldots, n_{k}^{0}\right)$ such that $\varphi\left(n_{1}^{0}, \ldots, n_{k}^{0}\right)$ is not true. Let $R$ be the database that decodes $\left(n_{1}^{0}, \ldots, n_{k}^{0}\right)$. The result of $Q_{\varphi}(R)$ will be the origin $(0,0)$ of $\mathbf{R} \times \mathbf{R}$. If we now apply $f_{0}$ to this result, the output is a vector $(y, z) \neq(0,0)$. On the other side, if we first apply $f_{0}$ to $R$, there are three possibilities. Either $f_{0}(R)$ encodes a vector $\left(n_{1}^{1}, \ldots, n_{k}^{1}\right)$ for which $\varphi\left(n_{1}^{1}, \ldots, n_{k}^{1}\right)$ is true, then the result of $Q_{\varphi}\left(f_{0}(R)\right)$ will be $\emptyset$. Or, $f_{0}(R)$ encodes a vector $\left(n_{1}^{1}, \ldots\right.$, $\left.n_{k}^{1}\right)$ for which $\varphi\left(n_{1}^{1}, \ldots, n_{k}^{1}\right)$ is not true, and $Q_{\varphi}\left(f_{0}(R)\right)$ returns $(0,0)$. In the last case, $f_{0}(R)$ does not encode a vector of natural numbers, in which case the result of $Q_{\varphi}\left(f_{0}(R)\right)$ will be $\emptyset$ again. In all cases, we have that $Q_{\varphi}\left(f_{0}(R)\right) \neq f_{0}\left(Q_{\varphi}(R)\right)$. Therefeore, the query $Q_{\varphi}$ is not $\mathcal{F}$-generic.

We can conclude that $Q_{\varphi}$ is $\mathcal{F}$-generic if and only if the sentence $\left(\forall n_{1}\right) \cdots\left(\forall n_{k}\right)$ $\varphi\left(n_{1}, \ldots, n_{k}\right)$ holds in the natural numbers.

Therefore, if $\mathcal{F}$-genericity would be decidable, also the truth of sentences in the $\forall^{*}$-fragment of number theory would be decidable. This concludes the proof.

In the remainder of this section, we show that the first-order queries that are $\left(\mathcal{F}_{s t}, \mathcal{F}_{t}\right)$-generic are recursively enumerable, however. We show this by giving sound and complete languages for the $\left(\mathcal{F}_{s t}, \mathcal{F}_{t}\right)$-generic FO-queries, for the groups $\left(\mathcal{F}_{s t}, \mathcal{F}_{t}\right)$ mentioned in Section 3.

We first define these sound and complete languages that are point-based logics. 
Definition 4.1. Let $\sigma=\left\{R_{1}, R_{2}, \ldots, R_{m}\right\}$ be a spatio-temporal database schema and let $\Pi$ be a set of predicates. The first-order logic over $\sigma$ and $\Pi$, denoted by $\mathrm{FO}\left(\Pi, R_{1}, R_{2}, \ldots, R_{m}\right)$ or $\mathrm{FO}(\Pi, \sigma)$, can be used as a spatio-temporal query language when variables are interpreted to range over points in $\mathbf{R}^{n} \times \mathbf{R}$, (we denote variables by $u, v, w, \ldots)$. The atomic formulas in $\mathrm{FO}(\Pi, \sigma)$ are equality constraints on point variables, the predicates of $\Pi$ applied to point variables, and the relation names $R_{1}, R_{2}, \ldots, R_{m}$ from $\sigma$ applied to point variables.

A $\mathrm{FO}(\Pi, \sigma)$-formula $\varphi\left(v_{1}, v_{2}, \ldots, v_{k}\right)$ defines for each spatio-temporal database

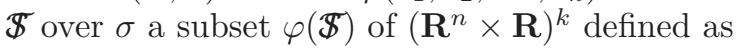

$$
\left\{\left(p_{1}, \ldots, p_{k}\right) \in\left(\mathbf{R}^{n} \times \mathbf{R}\right)^{k} \mid\left(\mathbf{R}^{n} \times \mathbf{R}, \Pi^{\mathbf{R}^{n} \times \mathbf{R}}, R_{1}^{\mathbb{\Phi}}, \ldots, R_{m}^{\mathbb{\Phi}}\right) \models \varphi\left[p_{1}, \ldots, p_{k}\right]\right\},
$$

where $\varphi\left[p_{1}, \ldots, p_{k}\right]$ is obtained from the formula $\varphi\left(v_{1}, \ldots, v_{k}\right)$ by instantiating the variables $v_{i}$ by the constant points $p_{i}, 1 \leq i \leq k$.

From Definition 2.7, it is clear what it means that a $\mathrm{FO}(\Pi, \sigma)$-formula expresses a semi-algebraic databases query.

Definition 4.2. A query language is said to be sound for the $\left(\mathcal{F}_{s t}, \mathcal{F}_{t}\right)$-generic $\mathrm{FO}(+, \times,<, 0,1)$-queries on spatio-temporal databases, if that language only expresses $\left(\mathcal{F}_{s t}, \mathcal{F}_{t}\right)$-generic $\mathrm{FO}(+, \times,<, 0,1)$-queries on spatio-temporal databases.

A query language is said to be complete for the $\left(\mathcal{F}_{s t}, \mathcal{F}_{t}\right)$-generic $\mathrm{FO}(+, \times,<, 0$, $1)$-queries on spatio-temporal databases, if all $\left(\mathcal{F}_{s t}, \mathcal{F}_{t}\right)$-generic $\mathrm{FO}(+, \times,<, 0,1)$ queries on spatio-temporal databases can be expressed in that language.

In the following, we will omit the dependence on the input schema when this is clear from the context, and use the notation $\mathrm{FO}(\Pi)$ for first-order point languages over the predicate set $\Pi$.

In the remainder of this section, we first discuss notions of genericity determined by time-independent transformations (Section 4.1), afterwards we discuss applications to physics (Section 4.2) and we end with genericity for the time-dependent transformations (Section 4.3).

\subsection{Genericity for time-independent transformations}

In this section, we give a general result concerning $\left(\mathcal{F}_{s t}, \mathcal{F}_{t}\right)$-generic queries where $\mathcal{F}_{s t}$ is a subgroup of $\mathcal{A}_{s t}^{c}$, the group of time-independent affinities of $\mathbf{R}^{n} \times \mathbf{R}$. First, we introduce the point predicates that we will use for the different point languages.

To express the temporal order of events, we use the point predicate Before. Let $p_{1}$ and $p_{2}$ be points in $\mathbf{R}^{n} \times \mathbf{R}$. The expression $\operatorname{Before}\left(p_{1}, p_{2}\right)$ evaluates to true if the time coordinate $\tau_{1}$ of $p_{1}$ is smaller than or equal to the time coordinate $\tau_{2}$ of $p_{2}$. In the following, we will often use the derived binary predicate Cotemp, which expresses for two points $p_{1}$ and $p_{2}$ that $\tau_{1}$ equals $\tau_{2}$. This predicate can be expressed in terms of Before as follows:

$$
\operatorname{Cotemp}(u, v):=\operatorname{Before}(u, v) \wedge \operatorname{Before}(v, u) .
$$

There are three more other purely temporal predicates: UnitTime, $\mathbf{0}_{\mathbf{t}}$ and $\mathbf{1}_{\mathbf{t}}$. The predicate UnitTime $\left(p_{1}, p_{2}\right)$ expresses that the points $p_{1}, p_{2} \in \mathbf{R}^{n} \times \mathbf{R}$ have time-coordinates $\tau_{1}$ and $\tau_{2}$ such that $\left|\tau_{1}-\tau_{2}\right|=1$. The unary predicates $\mathbf{0}_{\mathbf{t}}$ and $\mathbf{1}_{\mathbf{t}}$ 
are such that $\mathbf{0}_{\mathbf{t}}(p)$ and $\mathbf{1}_{\mathbf{t}}(p)$ respectively express that the time coordinate of the point $p$ equals to zero and to one.

The following predicates address spatio-temporal relations between points. The point-predicate Between ${ }^{n+1}$ is defined such that Between $^{n+1}\left(p_{1}, p_{2}, p_{3}\right)$ expresses that the points $p_{1}, p_{2}, p_{3}$ in $\mathbf{R}^{n} \times \mathbf{R}$ are collinear (in the space $\mathbf{R}^{n} \times \mathbf{R}$ ) and that $p_{2}$ is between $p_{1}$ and $p_{3}$. The predicates $\leq_{i}\left(p_{1}, p_{2}\right)(1 \leq i \leq n)$ express that the $i$ th spatial coordinate of $p_{1}$ is less or equal than the $i$ th spatial coordinate of $p_{2}$. The expression $\mathbf{E q D i s t}\left(p_{1}, p_{2}, p_{3}, p_{4}\right)$ is true if the distance between the two co-temporal points $p_{1}$ and $p_{2}$ equals the distance between the two co-temporal points $p_{3}$ and $p_{4}$. The binary predicate UnitDist applied to two points $p_{1}$ and $p_{2}$ expresses that they are co-temporal and that the (spatial) distance between $p_{1}$ and $p_{2}$ equals one. Finally, $\operatorname{Pos}^{n+1}\left(p_{0}, p_{1}, p_{2}, \ldots, p_{n+1}\right)$ expresses that the $(n+1)$ tuple $\left(p_{0}, p_{1}, p_{2}, \ldots, p_{n+1}\right)$ of points in $\mathbf{R}^{n} \times \mathbf{R}$ forms a positively oriented $(n+1)$ dimensional coordinate system with $p_{0}$ as origin.

Property 4.1. The point predicates Before, Between ${ }^{n+1}$, UnitTime, $\mathbf{0}_{\mathbf{t}}$, $\mathbf{1}_{\mathbf{t}}, \leq_{i}(1 \leq i \leq n)$, EqDist, UnitDist and $\mathbf{P o s}^{n+1}$ are all expressible in $\mathrm{FO}(+, \times$, $<, 0,1)$.

Proof. The $\mathrm{FO}(+, \times,<, 0,1)$-formulas for the different predicates can be obtained by expressing the constraints on the coordinates of the points satisfying the predicates. We denote the coordinates of a point variable $v_{i}$ in $\mathbf{R}^{n} \times \mathbf{R}$ by $\left(x_{i_{1}}, x_{i_{2}}, \ldots, x_{i_{n}}, t_{i}\right), \quad i=1,2, \ldots$.

The translation of the expression $\operatorname{Before}\left(v_{1}, v_{2}\right)$ is $t_{1} \leq t_{2}$ and the translation of UnitTime $\left(v_{1}, v_{2}\right)$ equals $\left|t_{1}-t_{2}\right|=1$. The translation of $\mathbf{0}_{\mathbf{t}}$ and $\mathbf{1}_{\mathbf{t}}$ is straightforward.

It is well known (e.g. [Gyssens et al. 1999]) that the predicates Between ${ }^{n+1}$, $\leq_{i}(1 \leq i \leq n)$, EqDist and UnitDist are expressible in $\mathrm{FO}(+, \times,<, 0,1)$. For EqDist and UnitDist it is necessary to use Before to express that their arguments should be co-temporal.

The expression $\operatorname{Pos}^{n+1}\left(v_{0}, v_{1}, \ldots, v_{n+1}\right)$ is translated into FO by expressing that the vectors $v_{1}-v_{0}, \ldots, v_{n+1}-v_{0}$ are linearly independent and that the $(n+1) \times$ $(n+1)$-matrix containing their coordinates has a strictly positive determinant.

\begin{tabular}{|l|l|}
\hline \hline$\left(\mathcal{F}_{s t}, \mathcal{F}_{t}\right)$ & Sets of point predicates $\Pi\left(\mathcal{F}_{s t}, \mathcal{F}_{t}\right)$ \\
\hline \hline$\left(\mathcal{A}_{s t}^{c}, \mathcal{A}_{t}\right)$ & $\left\{\right.$ Between $^{n+1}$, Before $\}$ \\
$\left(\mathcal{A}_{s t}^{c}, \mathcal{T}_{t}\right)$ & $\left\{\right.$ Between $^{n+1}$, Before, UnitTime $\}$ \\
$\left(\mathcal{A}_{s t}^{c}, \mathrm{Id}_{t}\right)$ & $\{$ Between \\
$\left(\mathcal{S}_{s t}^{c}, \mathcal{F}_{t}\right)$ & $\Pi\left(\mathcal{A}_{s t}^{c}, \mathcal{F}_{t}\right) \cup\{$ EqDist $\}$ \\
$\left(\mathcal{I}_{s t}^{c}, \mathcal{F}_{t}\right)$ & $\Pi\left(\mathcal{A}_{s t}^{c}, \mathcal{F}_{t}\right) \cup\{$ EqDist, UnitDist $\}$ \\
$\left(\mathcal{T}_{s t}^{c}, \mathcal{F}_{t}\right)$ & $\Pi\left(\mathcal{A}_{s t}^{c}, \mathcal{F}_{t}\right) \cup\left\{\right.$ EqDist, UnitDist $\left., \leq_{i}(1 \leq i \leq n), \mathbf{P o s}^{n+1}\right\}$ \\
\hline
\end{tabular}

Table I. An overview of the different sets of point predicates for a number of spatio-temporal genericity notions. In the three last cases $\mathcal{F}_{t} \in\left\{\mathcal{A}_{t}, \mathcal{T}_{t}, I d_{t}\right\}$.

Property 4.2. Let $\left(\mathcal{F}_{s t}, \mathcal{F}_{t}\right)$ be a group and let $\Pi\left(\mathcal{F}_{s t}, \mathcal{F}_{t}\right)$ be a set of point predicates as in Table I. The point predicates in $\Pi\left(\mathcal{F}_{s t}, \mathcal{F}_{t}\right)$ are invariant under elements of $\left(\mathcal{F}_{s t}, \mathcal{F}_{t}\right)$. 
Proof. First, remark that, if we fix $\mathcal{F}_{t}$ to be one of $\left\{\mathcal{A}_{t}, \mathcal{T}_{t}, I d_{t}\right\}$, then

$$
\left(\mathcal{T}_{s t}^{c}, \mathcal{F}_{t}\right) \subset\left(\mathcal{I}_{s t}^{c}, \mathcal{F}_{t}\right) \subset\left(\mathcal{S}_{s t}^{c}, \mathcal{F}_{t}\right) \subset\left(\mathcal{A}_{s t}^{c}, \mathcal{F}_{t}\right) .
$$

Also, if we fix $\mathcal{F}_{s t}$ to be one of $\left\{\mathcal{A}_{s t}^{c}, \mathcal{S}_{s t}^{c}, \mathcal{I}_{s t}^{c}, \mathcal{T}_{s t}^{c}\right\}$, then

$$
\left(\mathcal{F}_{s t}, I d_{t}\right) \subset\left(\mathcal{F}_{s t}, \mathcal{T}_{t}\right) \subset\left(\mathcal{F}_{s t}, \mathcal{A}_{t}\right)
$$

Also, all groups $\left(\mathcal{F}_{s t}, \mathcal{F}_{t}\right)$ are subgroups of the affinities of $\mathbf{R}^{n} \times \mathbf{R}$.

As we already remarked, if a point predicate is invariant for a certain transformation group $\left(\mathcal{F}_{s t}, \mathcal{F}_{t}\right)$, it is also invariant for all subgroups of $\left(\mathcal{F}_{s t}, \mathcal{F}_{t}\right)$.

We now prove invariance for each of the predicates in the sets $\Pi\left(\mathcal{F}_{s t}, \mathcal{F}_{t}\right)$ of Table I.

- The predicate Between ${ }^{n+1}$ is invariant under elements of $\left(\mathcal{A}_{s t}^{c}, \mathcal{A}_{t}\right)$. It is well known that affinities preserve the betweenness of points. As all groups listed in Table I are subgroups of the affinities of $\mathbf{R}^{n} \times \mathbf{R}$, the predicate Between $^{n+1}$ is invariant for all those groups.

- The predicate Before is invariant under elements of $\left(\mathcal{A}_{s t}^{c}, \mathcal{A}_{t}\right)$, since the elements of $\mathcal{A}_{t}$ are monotone bijections of time. As shown in Proposition 3.1, the order on time events is preserved under all strictly monotone increasing bijections of time. The groups $\mathcal{A}_{t}, \mathcal{I}_{t}, I d_{t}$ are all such bijections.

- The predicate UnitTime is invariant under elements of $\left(\mathcal{A}_{s t}^{c}, \mathcal{T}_{t}\right)$. It is straightforward that all elements of $\mathcal{T}_{t}$, which are translations in the time direction, preserve the time difference between any two points $p_{1}$ and $p_{2}$ in $\mathbf{R}^{n} \times \mathbf{R}$.

- The predicates $\mathbf{0}_{\mathbf{t}}$ and $\mathbf{1}_{\mathbf{t}}$ are invariant under elements of $\left(\mathcal{A}_{s t}^{c}, \mathrm{Id}_{t}\right)$. It is clear that the identity transformation on the time preserves the fact that a point $p$ in $\mathbf{R}^{n} \times \mathbf{R}$ has time coordinate zero or one.

- The predicate EqDist is invariant under elements of $\left(\mathcal{S}_{s t}^{c}, \mathcal{A}_{t}\right)$. It is well known that isometries and scalings (and thus similarities) preserve the fact that the distance between one pair of points equals the distance between a second pair of points. The groups $\mathcal{A}_{t}, \mathcal{T}_{t}, I d_{t}$ all preserve co-temporality of points.

- The predicate UnitDist is invariant under elements of $\left(\mathcal{I}_{s t}^{c}, \mathcal{A}_{t}\right)$, because isometries are distance preserving transformations.

- The predicates $\leq_{i}(1 \leq i \leq n)$ are invariant under elements of $\left(\mathcal{T}_{s t}^{c}, \mathcal{A}_{t}\right)$. It is easy to verify that if for two points $p_{1}$ and $p_{2}$ in $\mathbf{R}^{n} \times \mathbf{R}, \leq_{i}\left(p_{1}, p_{2}\right)$ is true for some $i$ in $\{1, \ldots, n\}$, also $\leq_{i}\left(f\left(p_{1}\right), f\left(p_{2}\right)\right)$ holds for each $f$ in $\left(\mathcal{T}_{s t}^{c}, \mathcal{F}_{t}\right)$, where $\mathcal{F}_{t}$ is one of $\mathcal{A}_{t}, \mathcal{T}_{t}, I d_{t}$.

- The predicate $\operatorname{Pos}^{n+1}$ is invariant under elements of $\left(\mathcal{T}_{s t}^{c}, \mathcal{A}_{t}\right)$, since translations are orientation-preserving transformations.

REMARK 4.1. From now, all results are valid for underlying dimension $n \geq 2$.

The following theorem follows directly from the proof of Theorem 5.5 [Gyssens et al. 1999].

Theorem 4.2. Let $\sigma$ be a spatio-temporal database schema. Let $\mathcal{F}$ be a subgroup of the affinities of $\mathbf{R}^{n} \times \mathbf{R}$. Let $\Pi$ be a set of point-predicates that contains Between $^{n+1}$. If the predicates in $\Pi$ are $\mathrm{FO}(+, \times,<, 0,1)$-expressible and invariant under the transformations of $\mathcal{F}$ and if the fact " $\left(v_{0}, v_{1}, \ldots, v_{n+1}\right)$ is the 
image of the standard coordinate system of $\mathbf{R}^{n} \times \mathbf{R}$ under some element $f$ of $\mathcal{F}$ " is expressible in $\mathrm{FO}(\Pi)$, then $\mathrm{FO}(\Pi, \sigma)$ is sound and complete for the $\mathcal{F}$-generic spatio-temporal database queries that are expressible in $\mathrm{FO}(+, \times,<, 0,1, \bar{\sigma})$.

We now prove the following theorem.

Theorem 4.3. Let $\sigma$ be a spatio-temporal database schema. Let $\mathcal{F}_{s t}$ be a subgroup of $\mathcal{A}_{s t}^{c}$ and $\mathcal{F}_{t}$ a subgroup of $\mathcal{A}_{t}$. Let $\Pi\left(\mathcal{F}_{s t}, \mathcal{F}_{t}\right)$ be a set of point-predicates that contains Between ${ }^{n+1}$ and Before. If the predicates in $\Pi\left(\mathcal{F}_{s t}, \mathcal{F}_{t}\right)$ are FOexpressible and invariant under the transformations of $\left(\mathcal{F}_{s t}, \mathcal{F}_{t}\right)$ and if the fact " $\left(v_{0}\right.$, $\left.v_{1}, \ldots, v_{n+1}\right)$ is the image of the standard coordinate system under some element $f$ of $\left(\mathcal{F}_{s t}, \mathcal{F}_{t}\right)$ " is expressible in $\operatorname{FO}\left(\Pi\left(\mathcal{F}_{\text {st }}, \mathcal{F}_{\mathrm{t}}\right)\right)$, then the logic $\operatorname{FO}\left(\Pi\left(\mathcal{F}_{\text {st }}, \mathcal{F}_{\mathrm{t}}\right), \sigma\right)$ is sound and complete for the $\left(\mathcal{F}_{s t}, \mathcal{F}_{t}\right)$-generic spatio-temporal database queries that are expressible in $\mathrm{FO}(+, \times,<, 0,1, \bar{\sigma})$.

Proof. First, we show that the language $\mathrm{FO}\left(\left\{\right.\right.$ Between $^{n+1}$, Before $\left.\}\right)$ is sound and complete for the $\left(\mathcal{A}_{s t}^{c}, \mathcal{A}_{t}\right)$-generic $\mathrm{FO}(+, \times,<, 0,1, \bar{\sigma})$-expressible spatio-temporal database queries using Theorem 4.2. Indeed, it is clear that the group $\left(\mathcal{A}_{s t}^{c}, \mathcal{A}_{t}\right)$ is a subgroup of the affinities of $\mathbf{R}^{n} \times \mathbf{R}$. Furthermore, the expression $\operatorname{Before}(u, v)$, is expressible in $\mathrm{FO}(+, \times,<, 0,1)$ (see Property 4.1). Also, the predicates Between ${ }^{n+1}$ and Before are both invariant under elements of $\left(\mathcal{A}_{s t}^{c}, \mathcal{A}_{t}\right)$ (see Property 4.2).

To conclude this part of the proof, we need to show that there is an expression in $\mathrm{FO}\left(\left\{\right.\right.$ Between $^{n+1}$, Before $\left.\}\right)$ that, for $n+2$ arbitrary points $p_{0}, p_{1}$, $, \ldots, p_{n+1}$ in $\mathbf{R}^{n} \times \mathbf{R}$, states that $\left(p_{0}, p_{1}, \ldots, p_{n+1}\right)$ is the image of the standard coordinate system under some element $f$ of $\left(\mathcal{A}_{s t}^{c}, \mathcal{A}_{t}\right)$. It is known (e.g. [Gyssens et al. 1999; Schwabhäuser et al. 1983]) that there exists an expression in the language $\mathrm{FO}\left(\left\{\right.\right.$ Between $\left.\left.^{n+1}\right\}\right)$ that, for $n+2$ points $p_{0}, p_{1}, \ldots, p_{n+1}$ of $\mathbf{R}^{n} \times \mathbf{R}$, expresses that $\left(p_{0}, p_{1}, \ldots, p_{n+1}\right)$ is the image of the standard $(n+1)$-dimensional coordinate system under some affinity of $\mathbf{R}^{n} \times \mathbf{R}$. We refer to this expression as

$$
\operatorname{CoSys}_{\mathcal{A}}\left(v_{0}, v_{1}, \ldots, v_{n+1}\right) \text {. }
$$

Obviously, this formula also belongs to $\mathrm{FO}\left(\left\{\right.\right.$ Between $^{n+1}$, Before $\left.\}\right)$.

The expression for the image of the standard coordinate system under some element of $\left(\mathcal{A}_{s t}^{c}, \mathcal{A}_{t}\right)$ is as follows:

$$
\begin{gathered}
\operatorname{CoSys}_{\left(\mathcal{A}_{s t}^{c}, \mathcal{A}_{t}\right)}\left(v_{0}, v_{1}, \ldots, v_{n+1}\right):=\operatorname{CoSys}_{\mathcal{A}}\left(v_{0}, v_{1}, \ldots, v_{n+1}\right) \wedge \\
\bigwedge_{i=1}^{n} \operatorname{Cotemp}\left(v_{0}, v_{i}\right) \wedge \neg \operatorname{Before}\left(v_{n+1}, v_{0}\right) .
\end{gathered}
$$

It is easy to verify that any coordinate system that is an image of the standard coordinate system under an element of $\left(\mathcal{A}_{s t}^{c}, \mathcal{A}_{t}\right)$ satisfies this expression. Also, the reverse is true. For clarity, we show this only for $n=2$ (the general case is analogous).

Any coordinate system $\left(p_{0}, p_{1}, p_{2}, p_{3}\right)$ satisfying the expression $\operatorname{CoSys}_{\left(\mathcal{A}_{s t}^{c}, \mathcal{A}_{t}\right)}\left(v_{0}\right.$, $\left.v_{1}, v_{2}, v_{3}\right)$ is of the form $p_{0}=\left(a_{0,1}, a_{0,2}, \tau_{0}\right), p_{1}=\left(a_{1,1}, a_{1,2}, \tau_{0}\right), p_{2}=\left(a_{2,1}, a_{2,2}, \tau_{0}\right)$, $p_{3}=\left(a_{3,1}, a_{3,2}, \tau_{3}\right)$, where $\tau_{0}<\tau_{3}$ and the determinant 


$$
\left|\begin{array}{ccc}
a_{1,1}-a_{0,1} & a_{1,2}-a_{0,2} & 0 \\
a_{2,1}-a_{0,1} & a_{2,2}-a_{0,2} & 0 \\
a_{3,1}-a_{0,1} & a_{3,2}-a_{0,2} & \tau_{3}-\tau_{0}
\end{array}\right| \neq 0 .
$$

Now, we have to show that there exists an element $f$ of $\left(\mathcal{A}_{s t}^{c}, \mathcal{A}_{t}\right)$ such that the image of the standard coordinate system under $f$ equals $\left(p_{0}, p_{1}, p_{2}, p_{3}\right)$. As $\left(\mathcal{A}_{s t}^{c}, \mathcal{A}_{t}\right)$ is a subgroup of the affinities, $f$ is representable by a matrix. It is straightforward to derive that $f=\left(f_{s t}, f_{t}\right)$, where

$$
\begin{aligned}
& f_{s t}(x, y, t)=\left(\begin{array}{ll}
a_{1,1}-a_{0,1} & a_{2,1}-a_{0,1} \\
a_{1,2}-a_{0,2} & a_{2,2}-a_{0,2}
\end{array}\right)\left(\begin{array}{l}
x \\
y
\end{array}\right)+\left(\begin{array}{c}
\left(a_{3,1}-a_{0,1}\right) t+a_{0,1} \\
\left(a_{3,2}-a_{0,2}\right) t+a_{0,2}
\end{array}\right), \text { and } \\
& f_{t}(t)=\left(\tau_{3}-\tau_{0}\right) t+\tau_{0} .
\end{aligned}
$$

It is clear that $\left(\tau_{3}-\tau_{0}\right)>0$ and that, because of the inequality $(*)$, the value of the determinant $\left|\begin{array}{ll}a_{1,1}-a_{0,1} & a_{2,1}-a_{0,1} \\ a_{1,2}-a_{0,2} & a_{2,2}-a_{0,2}\end{array}\right|$ differs from zero, hence $f$ is an element of $\left(\mathcal{A}_{s t}^{c}, \mathcal{A}_{t}\right)$.

So far, we proved that the language $\operatorname{FO}\left(\left\{\right.\right.$ Between $^{n+1}$, Before $\left.\}, \sigma\right)$ is sound and complete for the $\left(\mathcal{A}_{s t}^{c}, \mathcal{A}_{t}\right)$-generic queries expressible in $\mathrm{FO}(+, \times,<, 0,1, \bar{\sigma})$. The fact that any other language $\operatorname{FO}\left(\Pi\left(\mathcal{F}_{\text {st }}, \mathcal{F}_{\mathrm{t}}\right), \sigma\right)$, where $\Pi\left(\mathcal{F}_{s t}, \mathcal{F}_{t}\right)$ contains Between $^{n+1}$ and Before, is sound and complete for the $\left(\mathcal{F}_{s t}, \mathcal{F}_{t}\right)$-generic $\mathrm{FO}(+$, $\times,<, 0,1, \bar{\sigma})$-queries for each subgroup $\left(\mathcal{F}_{s t}, \mathcal{F}_{t}\right)$ of $\left(\mathcal{A}_{s t}^{c}, \mathcal{A}_{t}\right)$, under the conditions stated in Theorem 4.3, follows from Theorem 4.2 together with the first part of this proof.

TheOREM 4.4. Let $\sigma$ be a spatio-temporal database schema. Let $\left(\mathcal{F}_{s t}, \mathcal{F}_{t}\right)$ be a group and let $\Pi\left(\mathcal{F}_{s t}, \mathcal{F}_{t}\right)$ be as in Table I. The point language $\operatorname{FO}\left(\Pi\left(\mathcal{F}_{s t}, \mathcal{F}_{t}\right)\right.$, $\sigma)$ is sound and complete for the $\left(\mathcal{F}_{s t}, \mathcal{F}_{t}\right)$-generic queries expressible in $\mathrm{FO}(+, \times$, $<, 0,1, \bar{\sigma})$.

Proof. We can apply Theorem 4.3 for all groups in Table I, because they are all subgroups of $\left(\mathcal{A}_{s t}^{c}, \mathcal{A}_{t}\right)$. From Properties 4.1 and 4.2 , it follows that all predicates are expressible in $\mathrm{FO}(+, \times,<, 0,1)$ and that they are invariant under transformations of the appropriate groups. The only thing left to prove is that, for all groups $\left(\mathcal{F}_{s t}\right.$, $\left.\mathcal{F}_{t}\right)$ from Table I, and for $n+2$ points $v_{0}, v_{1}, \ldots, v_{n+1}$ in $\mathbf{R}^{n} \times \mathbf{R}$, the fact " $\left(v_{0}\right.$, $\left.v_{1}, \ldots, v_{n+1}\right)$ is the image of the standard coordinate system under some element $f$ of $\left(\mathcal{F}_{s t}, \mathcal{F}_{t}\right)$ " is expressible in $\operatorname{FO}\left(\Pi\left(\mathcal{F}_{s t}, \mathcal{F}_{t}\right)\right)$. For each group $\left(\mathcal{F}_{s t}, \mathcal{F}_{t}\right)$ from Table I, we now give a formula that expresses this fact. The correctness of these formulas is easy to verify.

- For the group $\left(\mathcal{A}_{s t}^{c}, \mathcal{A}_{t}\right)$, we already gave a formula in the proof of Theorem 4.3. The desired formula is there denoted by $\operatorname{CoSys}_{\left(\mathcal{A}_{s t}^{c}, \mathcal{A}_{t}\right)}$.

- For the group $\left(\mathcal{A}_{s t}^{c}, \mathcal{T}_{t}\right)$, we have

$$
\begin{aligned}
\operatorname{CoSys}_{\left(\mathcal{A}_{s t}^{c}, \mathcal{T}_{t}\right)}\left(v_{0},\right. & \left.v_{1}, \ldots, v_{n+1}\right):= \\
& \operatorname{CoSys}_{\left(\mathcal{A}_{s t}^{c}, \mathcal{A}_{t}\right)}\left(v_{0}, v_{1}, \ldots, v_{n+1}\right) \wedge \operatorname{UnitTime}\left(v_{0}, v_{n+1}\right) .
\end{aligned}
$$

ACM Journal Name, Vol. V, No. N, 20 YY. 
- For the group $\left(\mathcal{A}_{s t}^{c}, I d_{t}\right)$, we have

$$
\begin{aligned}
\operatorname{CoSys}_{\left(\mathcal{A}_{s t}^{c}, I d_{t}\right)}\left(v_{0}, v_{1}, \ldots, v_{n+1}\right):= & \\
& \operatorname{CoSys}_{\left(\mathcal{A}_{s t}^{c}, \mathcal{T}_{t}\right)}\left(v_{0}, v_{1}, \ldots, v_{n+1}\right) \wedge \mathbf{0}_{\mathbf{t}}\left(v_{0}\right) \wedge \mathbf{1}_{\mathbf{t}}\left(v_{n+1}\right) .
\end{aligned}
$$

Let $\mathcal{F}_{t}$ be an element of $\left\{\mathcal{A}_{t}, \mathcal{T}_{t}, I d_{t}\right\}$.

- For the groups $\left(\mathcal{S}_{s t}^{c}, \mathcal{F}_{t}\right)$, we have

$\operatorname{CoSys}_{\left(\mathcal{S}_{s t}^{c}, \mathcal{F}_{t}\right)}\left(v_{0}, v_{1}, \ldots, v_{n+1}\right):=$

$$
\operatorname{CoSys}_{\left(\mathcal{A}_{s t}^{c}, \mathcal{F}_{t}\right)}\left(v_{0}, v_{1}, \ldots, v_{n+1}\right) \wedge \bigwedge_{i=1}^{n} \bigwedge_{j=1}^{n} \operatorname{EqDist}\left(v_{0}, v_{i}, v_{0}, v_{j}\right) .
$$

- For the groups $\left(\mathcal{I}_{s t}^{c}, \mathcal{F}_{t}\right)$, we have

$$
\begin{aligned}
\operatorname{CoSys}_{\left(\mathcal{I}_{s t}^{c}, \mathcal{F}_{t}\right)}\left(v_{0}, v_{1}, \ldots, v_{n+1}\right):= \\
\operatorname{CoSys}_{\left(\mathcal{S}_{s t}^{c}, \mathcal{F}_{t}\right)}\left(v_{0}, v_{1}, \ldots, v_{n+1}\right) \wedge \bigwedge_{i=1}^{n} \operatorname{UnitDist}\left(v_{0}, v_{i}\right) .
\end{aligned}
$$

- For the groups $\left(\mathcal{T}_{s t}^{c}, \mathcal{F}_{t}\right)$, we have

$$
\begin{aligned}
\operatorname{CoSys}_{\left(\mathcal{T}_{s t}^{c}, \mathcal{F}_{t}\right)}\left(v_{0}, v_{1}, \ldots, v_{n+1}\right) & := \\
\operatorname{CoSys}_{\left(\mathcal{I}_{s t}^{c}, \mathcal{F}_{t}\right)}\left(v_{0}, v_{1}, \ldots, v_{n+1}\right) \wedge & \\
\operatorname{Pos}^{n+1}\left(v_{0}, v_{1}, \ldots, v_{n+1}\right) & \wedge \bigwedge_{j=1}^{n} \bigwedge_{i=1}^{n} \leq_{i}\left(v_{0}, v_{j}\right) .
\end{aligned}
$$

\subsection{Applications to Physics}

Here, we focus on the transformation groups $\left(\mathcal{V}_{s t}, \mathcal{T}_{t}\right),\left(\mathcal{V}(\mathcal{R})_{s t}, \mathcal{T}_{t}\right),\left(\mathcal{A C} \mathcal{C}_{s t}, \mathcal{T}_{t}\right)$ and $\left(\mathcal{A C}(\mathcal{R})_{s t}, \mathcal{T}_{t}\right)$. To formulate our results we need to define one more point-predicate, namely $=_{\text {space. }}$. If $p_{1}=\left(a_{1,1}, \ldots, a_{1, n}, \tau_{1}\right)$ and $p_{2}=\left(a_{2,1}, \ldots, a_{2, n}, \tau_{2}\right)$ are elements of $\mathbf{R}^{n} \times \mathbf{R}$, then $=_{\text {space }}\left(p_{1}, p_{2}\right)$ if and only if $a_{1, i}=a_{2, i}$ for all $1 \leq i \leq n$.

REMARK 4.2. The expression

$$
==_{\text {space }}\left(v_{1}, v_{2}\right):=\bigwedge_{i=1}^{n}\left(\leq_{i}\left(v_{1}, v_{2}\right) \wedge \leq_{i}\left(v_{2}, v_{1}\right)\right)
$$

is expressible in $\mathrm{FO}(+, \times,<, 0,1)$.

Theorem 4.5. Let $\sigma$ be a spatio-temporal database schema. Let the groups $\left(\mathcal{F}_{s t}, \mathcal{T}_{t}\right)$ and the predicate sets $\Pi\left(\mathcal{F}_{s t}, \mathcal{T}_{t}\right)$ be as in Table II. The point language $\mathrm{FO}\left(\Pi\left(\mathcal{F}_{s t}, \mathcal{T}_{t}\right), \sigma\right)$ is sound and complete for the $\left(\mathcal{F}_{s t}, \mathcal{T}_{t}\right)$-generic spatio-temporal queries that are expressible in $\mathrm{FO}(+, \times,<, 0,1, \bar{\sigma})$.

Proof. The transformation groups $\left(\mathcal{F}_{s t}, \mathcal{T}_{t}\right)$ of Table II are all subgroups of the group $\left(\mathcal{A}_{s t}^{c}, \mathcal{A}_{t}\right)$. Furthermore, the predicates of $\Pi\left(\mathcal{F}_{s t}, \mathcal{T}_{t}\right)$ are expressible in $\mathrm{FO}(+$, $\times,<, 0,1$ ) (see Property 4.1 and Remark 4.2). Straightforward geometrical and physical arguments show that all predicates are invariant under the appropriate transformation groups. We can now apply Theorem 4.3. We only have to verify that 


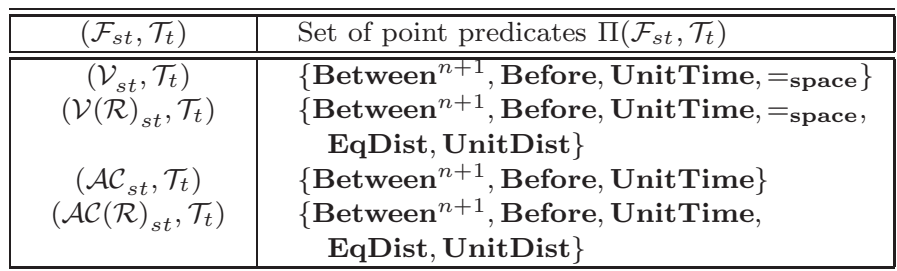

Table II. An overview of the different point-predicate sets for the physical transformation groups.

it is possible to express in the languages $\operatorname{FO}\left(\Pi\left(\mathcal{F}_{s t}, \mathcal{T}_{t}\right)\right)$ that a coordinate system is the image of the standard $(n+1)$-dimensional coordinate system under an element of $\left(\mathcal{F}_{s t}, \mathcal{T}_{t}\right)$. We now give, for each group $\left(\mathcal{F}_{s t}, \mathcal{F}_{t}\right)$ from Table II, the expression for the fact that $\left(v_{0}, v_{1}, \ldots, v_{n+1}\right)$ is the image of the standard coordinate system under some element $f$ of $\left(\mathcal{F}_{s t}, \mathcal{F}_{t}\right)$. The correctness of these expressions is easy to verify.

- For the group $\left(\mathcal{V}_{s t}, \mathcal{T}_{t}\right)$, we have

$$
\begin{aligned}
\operatorname{CoSys}_{\left(\mathcal{V}_{s t}, \mathcal{T}_{t}\right)}\left(v_{0}, v_{1}, \ldots, v_{n+1}\right):= \\
\operatorname{CoSys}_{\left(\mathcal{A}_{s t}, \mathcal{T}_{t}\right)}\left(v_{0}, v_{1}, \ldots, v_{n+1}\right) \wedge==_{\text {space }}\left(v_{0}, v_{n+1}\right),
\end{aligned}
$$

because elements of this group map the origin $(0, \ldots, 0,0)$ and the unit vector in the time-direction $(0, \ldots, 0,1)$ of the standard coordinate system of $\mathbf{R}^{n} \times \mathbf{R}$ onto points which have equal spatial coordinates.

- For the group $\left(\mathcal{V}(\mathcal{R})_{s t}, \mathcal{T}_{t}\right)$, we have

$$
\begin{aligned}
\operatorname{CoSys}_{\left(\mathcal{V}(\mathcal{R})_{s t}, \mathcal{T}_{t}\right)}\left(v_{0}, v_{1}, \ldots, v_{n+1}\right):= \\
\operatorname{CoSys}_{\left(\mathcal{I}_{s t}, \mathcal{T}_{t}\right)}\left(v_{0}, v_{1}, \ldots, v_{n+1}\right) \wedge={ }_{\text {space }}\left(v_{0}, v_{n+1}\right) .
\end{aligned}
$$

- For the group $\left(\mathcal{A C}_{s t}, \mathcal{T}_{t}\right)$, we have

$$
\operatorname{CoSys}_{\left(\mathcal{A C}_{s t}, \mathcal{T}_{t}\right)}\left(v_{0}, v_{1}, \ldots, v_{n+1}\right):=\operatorname{CoSys}_{\left(\mathcal{A}_{s t}, \mathcal{T}_{t}\right)}\left(v_{0}, v_{1}, \ldots, v_{n+1}\right) .
$$

- For the group $\left(\mathcal{A C}(\mathcal{R})_{s t}, \mathcal{T}_{t}\right)$, we have

$$
\operatorname{CoSys}_{\left(\mathcal{A C}(\mathcal{R})_{s t}, \mathcal{T}_{t}\right)}\left(v_{0}, v_{1}, \ldots, v_{n+1}\right):=\operatorname{CoSys}_{\left(\mathcal{I}_{s t}, \mathcal{T}_{t}\right)}\left(v_{0}, v_{1}, \ldots, v_{n+1}\right) .
$$

Next, we illustrate the languages summarized in Table I and Table II on the appropriate examples of Section 3.5.

ExAmple 4.1. We give the $\mathrm{FO}\left(\left\{\right.\right.$ Between $^{n+1}$, Before, UnitTime,$\left.\left.=_{\text {space }}\right\}\right)$ query $Q_{2}^{\prime}$ equivalent to the $\left(\mathcal{V}_{s t}, \mathcal{T}_{t}\right)$-generic query of Example 3.2: Give the places and time moments where car $A$ is standing still at that spot for at least 300 more seconds.

Remember that we assumed before that time is measured in seconds and distance is measured in meters. We first remark that the fact that one point is a constant number of seconds before another, can be expressed using UnitTime and Before. We illustrate this for an easy example where one point is 3 seconds after another:

$$
3 \sec (u, v):=\left(\exists w_{1}\right)\left(\exists w_{2}\right)\left(\operatorname{Before}\left(u, w_{1}\right) \wedge \operatorname{Before}\left(w_{1}, w_{2}\right) \wedge \operatorname{Before}\left(w_{2}, v\right) \wedge\right.
$$




\section{Complete Query Languages for Spatio-Temporal Databases \\ $\left.\operatorname{UnitTime}\left(u, w_{1}\right) \wedge \operatorname{UnitTime}\left(w_{1}, w_{2}\right) \wedge \operatorname{UnitTime}\left(w_{2}, v\right)\right)$.}

Now we give the expression for $Q_{2}^{\prime}$ :

$$
\begin{aligned}
\operatorname{car} A(u) & \wedge(\exists v)(300 \sec (u, v) \wedge \\
(\forall w)((\operatorname{Before}(u, w) & \left.\left.\wedge \operatorname{Before}(w, v) \wedge \operatorname{car} A(w)) \rightarrow==_{\mathbf{s p a c e}}(u, w)\right)\right) .
\end{aligned}
$$

Example 4.2. We give the $\mathrm{FO}\left(\left\{\right.\right.$ Between $^{n+1}$, Before, UnitTime $\left.\}\right)$-query $Q_{5}^{\prime}$ equivalent to the $\left(\mathcal{A C}_{s t}, \mathcal{T}_{t}\right)$-generic query of Example 3.5: Did car $A$ encounter any empty roads? I.e., were there parts of its trajectory where it could drive at constant speed for at least 6000 seconds.

$$
\begin{aligned}
(\exists u)(\exists v)(\operatorname{car} A(u) & \wedge \operatorname{car} A(v) \\
\operatorname{Before}(u, w) & \left.\left.\wedge \operatorname{Before}(w, v)) \rightarrow\left(\operatorname{Between}^{n+1}(u, v, w)\right)\right)\right) .
\end{aligned}
$$

\subsection{Genericity for time-dependent transformations}

Here, we focus on notions of genericity determined by time-dependent transformations. Our first result in this context shows that we can restrict our attention, without loss of generality, to piece-wise constant transformations.

Proposition 4.1. Let $Q$ be a spatio-temporal query expressible in $\mathrm{FO}(+, \times,<$, 0,1 ) and let the group $\mathcal{F}_{s t}$ be $\mathcal{A}_{s t}, \mathcal{S}_{s t}, \mathcal{I}_{s t}$ or $\mathcal{T}_{s t}$ and the group $\mathcal{F}_{t}$ be $\mathcal{A}_{t}$, $\mathcal{T}_{t}$ or $I d_{t}$. Then $Q$ is $\left(\mathcal{F}_{s t}, \mathcal{F}_{t}\right)$-generic if and only if it is $\left(\mathcal{F}_{s t}^{f}, \mathcal{F}_{t}\right)$-generic.

Although we postpone the proof of this proposition until the end of this section, it allows us to focus on subgroups of $\left(\mathcal{A}_{s t}^{f}, \mathcal{A}_{t}\right)$.

We first look at the group $\left(\mathcal{A}_{s t}^{f}, \mathcal{A}_{t}\right)$ and next on its subgroups. It will become clear later, that the proof strategy for these groups is analogous to that for the $\operatorname{group}\left(\mathcal{A}_{s t}^{f}, \mathcal{A}_{t}\right)$.

It is important to note that for $\left(\mathcal{A}_{s t}^{f}, \mathcal{A}_{t}\right)$ and its subgroups, we cannot apply Theorem 4.3. Indeed, it heavily relies on the fact that, using the predicate Between $^{n+1}$, it can be expressed that $n+2$ points form an affine coordinate system for the space $\mathbf{R}^{n} \times \mathbf{R}$, and also that some points represent the coordinates of another point, relative to such an affine coordinate system (the latter is a straightforward consequence of the former). When using the transformation group $\left(\mathcal{A}_{s t}^{f}, \mathcal{A}_{t}\right)$ or one of its subgroups, the predicate Between ${ }^{n+1}$ is too strong. Indeed, transformations of the group $\left(\mathcal{A}_{s t}^{f}, \mathcal{A}_{t}\right)$ do not preserve "betweenness" in $(n+1)$-dimensional space of points with different time coordinates. Therefore, the notion of collinearity in $(n+1)$-dimensional space can no longer be used. Figure 2 illustrates this with a line (left) and the image of the line under some transformation $\alpha=\left(\alpha_{s t}, \alpha_{t}\right)$ in $\left(\mathcal{A}_{s t}^{f}, \mathcal{A}_{t}\right)$ for which $\alpha_{t}$ is the identity function and $\alpha_{s t}$ equals the identity in the time interval $\left[t_{0}, t_{b}\right.$ [ and is a constant translation of space for the interval $\left[t_{b}, t_{1}\right]$. In the left part of Figure 2 , it is true that all points different from the endpoints at time moments $t_{0}$ and $t_{1}$ lie between the endpoints. For the right part of Figure 2 this is not true (the dashed line connecting the end points indicates all points between them.) 


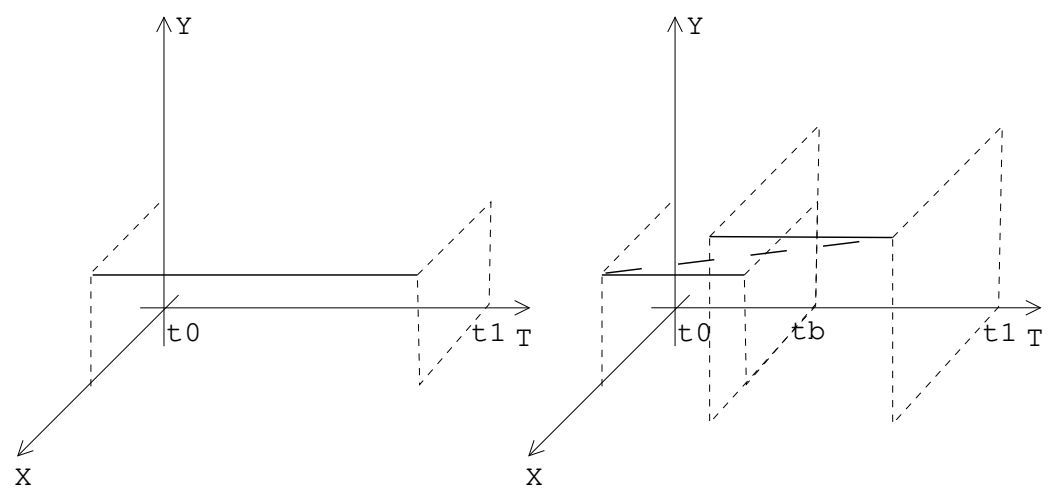

Fig. 2. The elements of $\left(\mathcal{A}_{s t}^{f}, \mathcal{A}_{t}\right)$ do not preserve betweenness of points.

However, as we want our language to be able to express all first-order $\left(\mathcal{A}_{s t}^{f}, \mathcal{A}_{t}\right)$ generic queries, somehow there needs to be a link between an $(n+1)$-dimensional point and its coordinates. It will become more clear later that, although we cannot express projection along the time axis, this link can be expressed using the predicates Between ${ }^{n}$, Before and a new predicate, $\mathbf{E q C R}^{\text {st }}$.

The predicate Before has already been introduced in Section 4.1. The expression Between $^{n}(p, q, r)$ states, for three points $p, q, r \in \mathbf{R}^{n} \times \mathbf{R}$, that they are co-temporal, collinear in the space $\mathbf{R}^{n}$ and that $q$ is between $p$ and $r$. We also

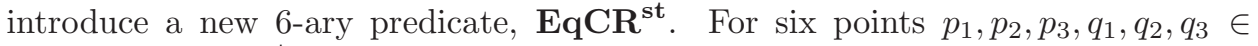

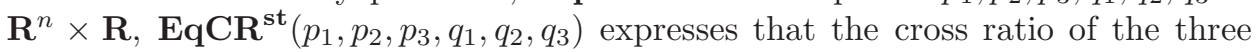
co-temporal and collinear points $p_{1}, p_{2}$ and $p_{3}$ equals the cross ratio of the time coordinates $\tau_{q_{1}}, \tau_{q_{2}}$ and $\tau_{q_{3}}$ of the points $q_{1}, q_{2}$ and $q_{3}$. The cross ratio of three collinear points $p, q, r$ is $\frac{|p q|}{|p r|}$, where $|p q|$ denotes the length of the line segment between $p$ and $q$. It is well known that the cross ratio is invariant under affine transformations.

For example, in $\mathbf{R}^{2} \times \mathbf{R}$,

$$
\mathbf{E q C R}^{\mathbf{s t}}((0,0,0),(1,1,0),(2,2,0),(0,0,0),(0,0,1),(0,0,2))
$$

holds, since the former three points have a cross ratio of $\frac{\sqrt{2}}{2 \sqrt{2}}$ and the latter three points have a cross ratio of $\frac{1}{2}$.

For ease of use, we will often use the predicates $\mathbf{E q C R}$ for the cross-ratio of spatial coordinates, and $\mathbf{E q} \mathbf{C R}^{\mathbf{t}}$ for the cross-ratio of temporal coordinates. Both predicates can be expressed using $\mathbf{E q} \mathbf{C R}^{\text {st }}$ :

$$
\begin{aligned}
& \mathbf{E q C R}^{\mathbf{s}}\left(u_{1}, u_{2}, u_{3}, v_{1}, v_{2}, v_{3}\right):=\left(\exists w_{1}\right)\left(\exists w_{2}\right)\left(\exists w_{3}\right) \\
&\left(\mathbf{E q C R}^{\mathbf{s t}}\left(u_{1}, u_{2}, u_{3}, w_{1}, w_{2}, w_{3}\right) \wedge \mathbf{E q C R} \mathbf{C R}^{\mathbf{s t}}\left(v_{1}, v_{2}, v_{3}, w_{1}, w_{2}, w_{3}\right)\right),
\end{aligned}
$$

and

$$
\begin{aligned}
& \mathbf{E q C R}^{\mathbf{t}}\left(u_{1}, u_{2}, u_{3}, v_{1}, v_{2}, v_{3}\right):=\left(\exists w_{1}\right)\left(\exists w_{2}\right)\left(\exists w_{3}\right) \\
&\left(\mathbf{E q C R}^{\mathbf{s t}}\left(w_{1}, w_{2}, w_{3}, u_{1}, u_{2}, u_{3}\right) \wedge \mathbf{E q C R} \mathbf{C R}^{\mathbf{s t}}\left(w_{1}, w_{2}, w_{3}, v_{1}, v_{2}, v_{3}\right)\right) .
\end{aligned}
$$

ACM Journal Name, Vol. V, No. N, 20 YY. 
Next, we present the main theorem of this section. The proof is composed of three lemmas, as explained below.

Theorem 4.6. Let $\sigma$ be a spatio-temporal database schema. The language $\mathrm{FO}\left(\left\{\right.\right.$ Between $^{n}$, Before, $\left.\left._{\mathbf{E q C R}} \mathbf{C R}^{\text {st }}\right\}, \sigma\right)$ is sound and complete for the $\left(\mathcal{A}_{s t}^{f}, \mathcal{A}_{t}\right)$ generic spatio-temporal queries that are expressible in $\mathrm{FO}(+, \times,<, 0,1, \bar{\sigma})$.

For the remainder of this section, we will assume that $\Pi$ denotes the set $\left\{\right.$ Between $^{n}$, Before, $\mathbf{E q C R}^{\text {st }}$ \}, unless stated otherwise.

We prove this theorem by three lemmas. First, the soundness is addressed in Lemma 4.1. Next, we prove completeness in two steps: Lemma 4.2 shows that every $\mathrm{FO}(+, \times,<, 0,1, \bar{\sigma})$-formula can be converted into a $\mathrm{FO}(\Pi, \sigma)$-formula, parameterized by a set of coordinate systems and Lemma 4.3 shows then that every $\left(\mathcal{A}_{s t}^{f}, \mathcal{A}_{t}\right)$-generic spatio-temporal query that is expressible in $\mathrm{FO}(+, \times,<, 0$, 1) can be converted into an equivalent query expressible in the language $\mathrm{FO}(\Pi)$.

Lemma 4.1. Let $\sigma$ be a spatio-temporal database schema and let $n$ be the underlying dimension. The language $\mathrm{FO}(\Pi, \sigma)$ is sound for the $\left(\mathcal{A}_{s t}^{f}, \mathcal{A}_{t}\right)$ - generic spatio-temporal queries expressible in $\mathrm{FO}(+, \times,<, 0,1, \bar{\sigma})$.

Proof. Soundness is proved in two steps. First, we show that every $\mathrm{FO}(\Pi, \sigma)-$ formula is equivalently expressible in $\mathrm{FO}(+, \times,<, 0,1, \bar{\sigma})$ and afterwards that every $\mathrm{FO}(\Pi, \sigma)$-formula is invariant under elements of $\left(\mathcal{A}_{s t}^{f}, \mathcal{A}_{t}\right)$. Both are proved by induction on the structure of $\mathrm{FO}(\Pi, \sigma)$-formulas.

- Every $\mathrm{FO}(\Pi, \sigma)$-formula is expressible in $\mathrm{FO}(+, \times,<, 0,1, \bar{\sigma})$. The atomic formulas of $\mathrm{FO}(\Pi, \sigma)$ are equality on point variables, the predicates Between $^{n}$, Before, $\mathbf{E q C R}^{\text {st }}$ and formulas of the type $R\left(v_{1}, \ldots, v_{l}\right)$, where $R$ is a relation name from $\sigma$, with arity $l$. We now describe, for each of the above types of atomic formulas, how they can be translated into $\mathrm{FO}(+, \times,<, 0,1, \bar{\sigma})$.

A point variable $v$ occurring in a $\mathrm{FO}(\Pi, \sigma)$-formula is translated into real variables $x_{1}^{v}, \ldots, x_{n}^{v}, t^{v}$. Equality between two point variables is then expressed in $\mathrm{FO}(+, \times$, $<, 0,1, \bar{\sigma})$ by requiring that all corresponding coordinates of the two point variables are equal.

We already know that the predicate Before is expressible in $\mathrm{FO}(+, \times,<, 0,1)$. The predicate Between $^{n}$ is translated in a similar way as Between ${ }^{n+1}$, with the additional restriction that the time coordinates of the variables should be the same.

The formula $\mathbf{E q C R} \mathbf{C R}^{\text {st }}\left(u_{1}, u_{2}, u_{3}, v_{1}, v_{2}, v_{3}\right)$ is translated as the conjunction of the translation of the expression Collinear ${ }^{n}\left(u_{1}, u_{2}, u_{3}\right)$, which is equal to

$$
\operatorname{Between}^{n}\left(u_{1}, u_{2}, u_{3}\right) \vee \operatorname{Between}^{n}\left(u_{2}, u_{1}, u_{3}\right) \vee \operatorname{Between}^{n}\left(u_{1}, u_{3}, u_{2}\right)
$$

and the formula

$$
\left(t^{v_{3}}-t^{v_{1}}\right)^{2} \sum_{i=1}^{n}\left(x_{i}^{u_{1}}-x_{i}^{u_{2}}\right)^{2}=\left(t^{v_{2}}-t^{v_{1}}\right)^{2} \sum_{i=1}^{n}\left(x_{i}^{u_{1}}-x_{i}^{u_{3}}\right)^{2} .
$$

We translate formulas of the type $R\left(v_{1}, \ldots, v_{l}\right)$, where $R$ is a relation name from $\sigma$ with arity $l$, by the formula $\bar{R}\left(x_{1}^{v_{1}}, \ldots, x_{n}^{v_{1}}, t^{v_{1}}, \ldots, x_{1}^{v_{l}}, \ldots, x_{n}^{v_{l}}, t^{v_{l}}\right)$.

Compositions of atomic formulas by logical connectives and quantifiers are translated in a natural way. 
- Every $\mathrm{FO}(\Pi, \sigma)$ formula is invariant for elements of the group $\left(\mathcal{A}_{s t}^{f}, \mathcal{A}_{t}\right)$. The only non-trivial part here is showing that all point predicates are $\left(\mathcal{A}_{s t}^{f}, \mathcal{A}_{t}\right)$-invariant. The predicate Before is invariant for all transformations $f=\left(f_{1}, f_{2}, \ldots, f_{n}, f_{t}\right)$, that map $\mathbf{R}^{n} \times \mathbf{R}$ to $\mathbf{R}^{n} \times \mathbf{R}$, such that $f_{t}$ is a strictly monotone increasing bijection of $t$ alone (Proposition 3.1). Since all elements of $\mathcal{A}_{t}$ are such bijections, this condition is satisfied for $\left(\mathcal{A}_{s t}^{f}, \mathcal{A}_{t}\right)$. It is well known that affinities preserve the crossratio of three points. Because the predicate Between $^{n}$ requires its parameters to be co-temporal (which is preserved by elements of $\left(\mathcal{A}_{s t}^{f}, \mathcal{A}_{t}\right)$ ), these co-temporal points will be transformed by the same affinity and hence their cross-ratio is preserved. Also the predicate $\mathbf{E q C R}{ }^{\text {st }}$ is invariant under elements of $\left(\mathcal{A}_{s t}^{f}, \mathcal{A}_{t}\right)$, because the group $\mathcal{A}_{s t}^{f}$ preserves the cross-ratio between the spatial coordinates of co-temporal points and the group $\mathcal{A}_{t}$ preserves the cross-ratio between time coordinates.

We now show that every $\mathrm{FO}(+, \times,<, 0,1, \bar{\sigma})$-formula can be converted into a $\mathrm{FO}\left(\left\{\right.\right.$ Between $^{n}$, Before $\left.\left.\mathbf{E q C R}^{\text {st }}\right\}, \sigma\right)$ formula, which is parameterized by a finite set of coordinate systems.

A coordinate system in a $n$-dimensional hyperplane of $\mathbf{R}^{n} \times \mathbf{R}$, orthogonal to the time axis will be referred to as a spatial coordinate system and a coordinate system on the time-axis will be referred to as a temporal coordinate system.

If $p, q$ and $r$ are collinear points in $\mathbf{R}^{n} \times \mathbf{R}$, then we denote by $\frac{\overrightarrow{p q}}{\vec{p}}$ the real number $\alpha$ such that $\overrightarrow{p q}=\alpha \overrightarrow{p r}$.

Lemma 4.2. Let $\sigma$ be a spatio-temporal database schema and let the underlying dimension be $n$. For every $\mathrm{FO}(+, \times,<, 0,1, \bar{\sigma})$-formula

$$
\bar{\psi}\left(x_{1}, x_{2}, \ldots, x_{m}, t_{1}, \ldots, t_{l}\right),
$$

there exists a $\mathrm{FO}(\Pi, \sigma)$-formula

$$
\psi\left(u_{t_{O}}, u_{t_{E}}, u_{0,0}, u_{0,1}, \ldots, u_{0, n}, \ldots, u_{l, 0}, u_{l, 1}, \ldots, u_{l, n}, v_{1}, v_{2}, \ldots, v_{k}\right),
$$

where $l$ is the number of variablesoccurring in the formula that refer to a time dimension and where $k$ is the total number of free variables of $\bar{\psi}$, i.e., $k=m+l$. Furthermore, for each spatio-temporal database $\$$ over $\sigma$, for each set of spatial coordinate systems $\left(p_{i, 0}, p_{i, 1}, \ldots, p_{i, n}\right), i=0, \ldots, l$ of the spatial component of $\mathbf{R}^{n} \times \mathbf{R}$, for each temporal coordinate system $\left(p_{t_{O}}, p_{t_{E}}\right)$ of the temporal component of $\mathbf{R}^{n} \times \mathbf{R}$, and for all points $q_{1}, q_{2}, \ldots, q_{k}$ on the line $p_{0,0} p_{0,1}$ :

$$
\begin{aligned}
\left(\mathbf{R}^{n} \times \mathbf{R}, \Pi^{\mathbf{R}^{n} \times \mathbf{R}}, \Phi\right) & \models \psi\left[p_{t_{O}}, p_{t_{E}}, p_{0,0}, p_{0,1}, \ldots, p_{0, n}, \ldots,\right. \\
& \left.p_{l, 0}, p_{l, 1}, \ldots, p_{l, n}, q_{1}, q_{2}, \ldots, q_{k}\right]
\end{aligned}
$$

if and only if

$$
\left(\mathbf{R},+, \times,<, 0,1, \alpha(\bar{\Phi}) \models \bar{\psi}\left[\frac{\overrightarrow{p_{0,0} q_{1}}}{\overline{p_{0,0} p_{0,1}}}, \frac{\overrightarrow{p_{0,0} q_{2}}}{\overline{p_{0,0} p_{0,1}}}, \ldots, \frac{\overrightarrow{p_{0,0} q_{k}}}{\overline{\overline{p_{0,0} p_{0,1}}}}\right],\right.
$$

where $\alpha=\left(\alpha_{s t}, \alpha_{t}\right)$ is an element of $\left(\mathcal{A}_{s t}^{f}, \mathcal{A}_{t}\right)$ such that $\left(p_{0,0}, \ldots, p_{0, n}\right)$ is mapped by $\alpha_{s t}$ onto the standard spatial coordinate system in the hyperplane $\mathbf{R}^{n} \times\{(0, \ldots, 0,0)\}$ of $\mathbf{R}^{n} \times \mathbf{R}$, and each spatial coordinate system $\left(p_{i, 0}, p_{i, 1}, \ldots\right.$, $\left.p_{i, n}\right)(i=1 \ldots, l)$ is mapped on the standard coordinate system in the hyperplane at time $\mathbf{R}^{n} \times\left\{\alpha\left(\tau_{p_{i, 0}}\right)\right\}$ where the temporal part $\alpha_{t}$ of $\alpha$ is the unique time-affinity which maps $\tau_{p_{O}}$ to 0 and $\tau_{p_{E}}$ to 1 .

ACM Journal Name, Vol. V, No. N, $20 Y$ Y. 
Proof. Let $\bar{\psi}$ be a $\mathrm{FO}(+, \times,<, 0,1, \bar{\sigma})$-formula. We assume that $\bar{\psi}$ is in prenex normal form. We now describe the translation of $\bar{\psi}$ into a formula $\psi$ of $\mathrm{FO}(\Pi, \sigma)$ (by induction on its structure). In this translation, first the quantifier-free part of $\bar{\psi}$ is translated and the quantifiers are later added in the obvious way.

To start with, a 2-dimensional "computation plane" is chosen that is used to simulate real variables and constants and all the polynomial equations, polynomial equalities and inequalities.

- The choice of a computation plane. First of all, two moments in time $u_{t_{O}}$ and $u_{t_{E}}$ (time moments are simulated in $\psi$ by variables in $\mathbf{R}^{n} \times \mathbf{R}$ ) are chosen such that $\neg$ Before $\left(u_{t_{E}}, u_{t_{O}}\right)$. They form a temporal coordinate system; the formula describing this is as follows:

$$
\operatorname{TCoSys}_{\mathcal{A}_{t}}\left(u_{1}, u_{2}\right):=\neg \operatorname{Before}\left(u_{2}, u_{1}\right) .
$$

Next, in the hyperplane of points co-temporal with $u_{t_{O}}, n+1$ points $u_{0,0}, u_{0,1}, \ldots$ , $u_{0, n}$ are chosen such that they form an affine coordinate system for the hyperplane co-temporal with $u_{t_{O}}$. The predicate $\operatorname{CoSys}_{\mathcal{A}}^{n}$, expressing this, is similar the the previously introduced predicate $\operatorname{CoSys}_{\mathcal{A}}$ (see the proof of Theorem 4.4), except that some constraints are added that express that the points should be co-temporal.

As the variables $u_{t_{O}}, u_{t_{E}}, u_{0,0}, u_{0,1}, \ldots, u_{0, n}$ represent arbitrary points (up to the mentioned restrictions), they parameterise the translation of $\bar{\psi}$. To start with, $\psi$ will contain the subformula $\psi_{\text {comp }}$, defined as

$$
\begin{aligned}
\psi_{\text {comp }}\left(u_{t_{O}}, u_{t_{E}}, u_{0,0},\right. & \left.u_{0,1}, \ldots, u_{0, n}\right):=\operatorname{TCoSys}_{\mathcal{A}}\left(u_{t_{O}}, u_{t_{E}}\right) \\
& \wedge \operatorname{CoSys}_{\mathcal{A}}^{n}\left(u_{0,0}, u_{0,1}, \ldots, u_{0, n}\right) \wedge \operatorname{Cotemp}\left(u_{t_{O}}, u_{0,0}\right),
\end{aligned}
$$

as a conjunct.

We will use the 2-dimensional plane through the points $u_{0,0}, u_{0,1}$ and $u_{0,2}$ as a "computation plane". The idea is that we will simulate real variables and constants by points on the line through $u_{0,0}$ and $u_{0,1}$ and that addition and multiplication of real terms are simulated by $\mathrm{FO}(\Pi)$ expressions in the plane through $u_{0,0}, u_{0,1}$ and $u_{0,2}$.

- The translation of terms and atomic formulas. A quantifier-free $\mathrm{FO}(+, \times,<, 0,1$, $\bar{\sigma})$-formula may contain the following terms and atomic subformulas: real variables; the constants 0 and 1; polynomial constraints; and relation predicates where the relation names from $\bar{\sigma}$ are used. We translate each separately.

- The translation of real variables. Each real variable $x$ appearing in the formula $\bar{\psi}$ is translated into a spatio-temporal variable $v$. Also, $\psi$ will contain a conjunct

$$
\psi_{\text {var }}(v):=\text { Collinear }^{n}\left(u_{0,0}, u_{0,1}, v\right),
$$

expressing that $v$ is in the computation plane on the line connecting $u_{0,0}$ and $u_{0,1}$. The idea is that a real variable $x$ taking concrete value $a$, is simulated by requiring that $v$ is such that $\frac{\overrightarrow{u_{0,0} \vec{b}}}{\overline{u_{0,0} u_{0,1}}}$ equals $a$.

- The translation of the constants 0 and 1 . The real constants 0 and 1 that may appear in $\bar{\psi}$ are translated into $u_{0,0}$ and $u_{0,1}$ respectively.

- The translation of polynomial constraints. The arithmetic operations (addition and multiplication) on real terms will be simulated in the computation plane 
$\left(u_{0,0}, u_{0,1}, u_{0,2}\right)$ It was shown by Tarski [Schwabhäuser et al. 1983] (the results of Tarski were also used in [Gyssens et al. 1999]) that all arithmetic operations on points that are located on the line through $u_{0,0}$ and $u_{0,1}$ can be simulated in the plane $\left(u_{0,0}, u_{0,1}, u_{0,2}\right)$ using only the construct Between ${ }^{n}$.

Hence, a subformula $p\left(x_{1}, \ldots, x_{m}\right)>0$, with $p$ a polynomial with integer coefficients, using the translation of the real variables $x_{1}, \ldots, x_{m}$ in point variables $v_{1}, \ldots$, $v_{m}$, is translated into $\psi_{\text {poly }}\left(u_{0,0}, u_{0,1}, u_{0,2}, v_{1}, \ldots, v_{m}\right)$, defined using the predicate Between $^{n}$.

The correctness of the three above translations can be demonstrated as that of the similar translations in [Gyssens et al. 1999].

- The translation of relation predicates. A subformula of $\bar{\psi}$ of type $\bar{R}\left(x_{1,1}, \ldots\right.$, $\left.x_{1, n}, x_{1, t}, \ldots, x_{m, 1}, \ldots, x_{m, n}, x_{m, t}\right)$, where $\bar{R} \in \bar{\sigma}$ and where $m$ is the arity of $R$ in $\sigma$, is translated into a formula

$$
R\left(v_{1}, \ldots, v_{m}\right)
$$

and $\psi$ has a conjunct expressing that the point variables $v_{1,1}, \ldots, v_{1, n}, v_{1, t}, \ldots, v_{m, 1}$, $\ldots, v_{m, n}, v_{m, t}$, that are the translations of $x_{1,1}, \ldots, x_{1, n}, x_{1, t}, \ldots, x_{m, 1}, \ldots, x_{m, n}$, $x_{m, t}$, are the coordinates of $v_{1}, \ldots, v_{m}$ respectively. For the moment, we assume that the variables $x_{i, t}$ and $x_{j, t}$ are different for $1 \leq i<j \leq m$ and later show how to deal with the general case.

Indeed, recall that each variable $x_{i, j}(1 \leq i \leq m, 1 \leq j \leq n)$ and $x_{i, t}(1 \leq i \leq$ $m$ ) is already translated into a point variable $v_{i, j}$ and $v_{i, t}$, which are all collinear with $u_{0,0}$ and $u_{0,1}$. To express the link between the coordinates of point variables $v_{1}, \ldots, v_{m}$ and the point variables $v_{i, j}$ and $v_{i, t}$, we proceed as follows. We associate with each point variable $v_{i}(1 \leq i \leq m)$ the following set of point variables:

(1) $n+1$ point variables $u_{i, 0}, \ldots, u_{i, n}$ representing an $n$-dimensional coordinate system which is co-temporal with $v_{i}$; and

(2) $n$ point variables $v_{i, j}^{\prime}$ which are collinear with $u_{i, 0}$ and $u_{i, 1}$, such that $v_{i, j}^{\prime}$ represents the $j$ th coordinate of $v_{i}$ with respect to the coordinate systems specified by $u_{i, 0}, \ldots, u_{i, n}$, and such that the coordinate of $v_{i, j}^{\prime}$, on the line through $u_{i, 0}$ and $u_{i, 1}$, gives the same cross ratio with respect to these points as the coordinate of $v_{i, j}$, on the line through $u_{0,0}$ and $u_{0,1}$, gives with respect to these points, i.e., $\frac{\overrightarrow{u_{i, 0} v_{i, j}^{\prime}}}{\overline{u_{i, 0} u_{i, 1}}}=\frac{\overrightarrow{u_{i, 0} v_{i, j}}}{\overline{u_{0,0} u_{0,1}}}$.

As explained before, the first set of $n+1$ point variables can be defined using the expression

$$
\operatorname{CoSys}_{\mathcal{A}}^{n}\left(u_{i, 0}, u_{i, 1}, \ldots, u_{i, n}\right) \wedge \operatorname{Cotemp}\left(u_{i, 0}, v_{i}\right) .
$$

For the second set of $n$ point variables, we first observe that from [Gyssens et al. 1999], we know that we can express, using Between $^{n}$, that $n$ point variables $v_{i, 1}^{\prime}, \ldots, v_{i, n}^{\prime}$ represent the spatial coordinates of the point variable $v_{i}$ relative to a chosen spatial coordinate system (in this case, the coordinate system specified by $\left.u_{i, 0}, \ldots, u_{i, n}\right)$. In order to establish the link between the point variables $v_{i, j}^{\prime}$ in the plane specified by $u_{i, 0}, \ldots, u_{i, n}$ and the point variables $v_{i, j}$ in the computation plane we need to use the predicate $\mathbf{E q C R} \mathbf{C R}^{\mathbf{s}}$. The predicate $\mathbf{E q C R} \mathbf{C R}^{\mathbf{s}}$ performs a transformation between the affine coordinate systems at two different time moments, and so connects each $v_{i, j}^{\prime}$ to a $v_{i, j}(i=1, \ldots, m, j=1, \ldots, n)$. Remark that ACM Journal Name, Vol. V, No. N, $20 Y Y$. 
all $v_{i, j}^{\prime}$ are collinear with $u_{i, 0}$ and $u_{i, 1}$, and that all $v_{i, j}$ are collinear with $u_{0,0}$ and $u_{0,1}$. Therefore, $\mathbf{E q C R}$ can be used to express this equality of cross ratios.

Until now, we only considered the spatial coordinates. To link the temporal variables $v_{i, t}$ to the temporal coordinate of $v_{i}$, we use the expression $\mathbf{E q C R} \mathbf{C R}^{\mathrm{st}}\left(u_{0,0}\right.$, $\left.u_{0,1}, v_{i, t}, u_{t_{O}}, u_{t_{E}}, v_{i}\right)$. Recall that the predicate $\mathbf{E q C R} \mathbf{C R}^{\text {st }}$ can be used to relate the cross ratio of points on the time axis to the cross ratio of points, representing coordinates on the line through $u_{0,0}$ and $u_{0,1}$, and thus connects each $v_{i}$ to a $v_{i, t}$ $(i=1, \ldots, m)$.

Putting everything together results in the expression $\psi_{\text {rel }}$ :

$$
\begin{aligned}
&\left(\exists v_{1}\right)\left(\exists v_{2}\right) \ldots\left(\exists v_{m}\right)\left(R\left(v_{1}, v_{2}, \ldots, v_{m}\right) \wedge \bigwedge_{i=1}^{m} \operatorname{CoSys}_{\mathcal{A}}^{n}\left(u_{i, 0}, u_{i, 1}, \ldots, u_{i, n}\right)\right. \\
& \wedge \bigwedge_{i=1}^{m} \operatorname{Cotemp}^{m}\left(u_{i, 0}, v_{i}\right) \wedge\left(\exists v_{1,1}^{\prime}\right) \ldots\left(\exists v_{1, n}^{\prime}\right) \ldots\left(\exists v_{m, 1}^{\prime}\right) \ldots\left(\exists v_{m, n}^{\prime}\right) \\
&\left(\bigwedge_{i=1}^{m} \operatorname{Coordinates}^{n}\left(u_{i, 0}, u_{i, 1}, \ldots, u_{i, n}, v_{i, 1}^{\prime}, \ldots, v_{i, n}^{\prime}, v_{i}\right)\right.
\end{aligned}
$$$$
\wedge \bigwedge_{i=1}^{m} \bigwedge_{j=1}^{n} \operatorname{EqCR}^{\mathbf{s}}\left(u_{0,0}, u_{0,1}, v_{i, j}, u_{i, 0}, u_{i, 1}, v_{i, j}^{\prime}\right)
$$

$$
\left.\left.\wedge \bigwedge_{i=1}^{m} \operatorname{EqCR}^{\mathbf{s t}}\left(u_{0,0}, u_{0,1}, v_{i, t}, u_{t_{O}}, u_{t_{E}}, v_{i}\right)\right)\right)
$$

where Coordinates ${ }^{n}\left(u_{i, 0}, \ldots, u_{i, n}, v_{i, 1}^{\prime}, \ldots, v_{i, n}^{\prime}, v_{i}\right)$ expresses for each $(1 \leq j \leq n)$ that $v_{i, j}^{\prime}$ is represents the $j$ th coordinate of $v_{i}$ with respect to the coordinate systems specified by $u_{i, 0}, \ldots, u_{i, n}$.

We now show the correctness of the above translation of a relation predicate. We have to prove that for each spatio-temporal database $\mathbb{\$}$, and for any points $p_{t_{O}}, p_{t_{E}}, p_{0,0}, \ldots, p_{0, n}, \ldots, p_{m, 0}, \ldots, p_{m, n}, q_{1,1}, \ldots, q_{1, n}, q_{1, t}, \ldots, q_{m, 1}, \ldots, q_{m, n}, q_{m, t}$ :

$$
\begin{aligned}
\left(\mathbf{R}^{n} \times \mathbf{R}, \Pi^{\mathbf{R}^{n} \times \mathbf{R}}, \Phi\right) \models \psi_{\text {rel }}\left[p_{t_{O}}, p_{t_{E}}, p_{0,0}, \ldots, p_{0, n}, \ldots, p_{m, 0}, \ldots, p_{m, n},\right. \\
\left.q_{1,1}, \ldots, q_{1, n}, q_{1, t}, \ldots, q_{m, 1}, \ldots, q_{m, n}, q_{m, t}\right]
\end{aligned}
$$

if and only if

$$
\begin{aligned}
(\mathbf{R},+, \times, 0,1, \alpha(\overline{\boldsymbol{\Phi}})) \models \bar{R}\left[\frac{\overrightarrow{p_{0,0} q_{1,1}}}{\overrightarrow{p_{0,0} p_{0,1}}}, \ldots, \frac{\overrightarrow{p_{0,0} q_{1, n}}}{\overline{\overline{p_{0,0} p_{0,1}}}, \frac{\overrightarrow{p_{0,0} q_{1, t}}}{\overrightarrow{p_{0,0} p_{0,1}}}, \ldots,}\right. \\
\left.\frac{\overrightarrow{p_{0,0} q_{m, 1}}}{\overrightarrow{p_{0,0} p_{0,1}}}, \ldots, \frac{\overrightarrow{p_{0,0} q_{m, n}}}{\overrightarrow{p_{0,0} p_{0,1}}}, \frac{\overrightarrow{p_{0,0} q_{m, t}}}{\overrightarrow{p_{0,0} p_{0,1}}}\right],
\end{aligned}
$$

where $\alpha=\left(\alpha_{s t}, \alpha_{t}\right) \in\left(\mathcal{A}_{s t}^{f}, \mathcal{A}_{t}\right)$ is the affinity which maps $\left(p_{0,0}, \ldots, p_{0, n}\right)$ to the spatial standard basis at time $\tau_{0}=0,\left(p_{i, 0}, \ldots, p_{i, n}\right)$ to the spatial standard basis at time $\tau_{i}=\alpha\left(\tau_{p_{i, 0}}\right)$, where $\alpha_{t}$ is uniquely determined on the time axis by $\alpha_{t}\left(\tau_{p_{O}}\right)=0$ and $\alpha_{t}\left(p_{t_{E}}\right)=1$. Note that by assumption, $x_{i, t} \neq x_{j, t}$ for $(1 \leq i<j<m)$ and hence also $\tau_{p_{i, 0}}$ and $\tau_{p_{j, 0}}$, and consequently $\tau_{i} \neq \tau_{j}$ for $(1 \leq i<j<m)$. This condition is essential to ensure that $\alpha_{t}$ exists and is well defined. Indeed, suppose 
that there exists an $i$ and $j$ such that $\tau_{p_{i, 0}}=\tau_{p_{j, 0}}$ and hence $\tau_{i}=\tau_{j}$. Then we would require that $\alpha$ maps two possibly different co-temporal coordinate systems $\left(p_{i, 0}, \ldots, p_{i, n}\right)$ and $\left(p_{j, 0}, \ldots, p_{j, n}\right)$ the same standard basis. This can clearly not be done by a $\left(\mathcal{A}_{s t}^{f}, \mathcal{A}_{t}\right)$-generic query.

We know that the formula $\psi_{\text {rel }}$ is true for the points $p_{t_{O}}, p_{t_{E}}, p_{0,0}, \ldots, p_{0, n}, \ldots$, $p_{m, 0}, \ldots, p_{m, n}, q_{1,1}, \ldots, q_{1, n}, q_{1, t}, \ldots, q_{m, 1}, \ldots, q_{m, n}, q_{m, t}$ if and only if there exist points $p_{1}, \ldots, p_{m}, q_{1,1}^{\prime}, \ldots, q_{1, n}^{\prime}, \ldots, q_{m, 1}^{\prime}, \ldots, q_{m, n}^{\prime}$ such that for each $i=1, \ldots, m$ :

$$
\overrightarrow{p_{0,0} p_{i}}=\overrightarrow{p_{i, 0} p_{i}}+\overrightarrow{p_{0,0} p_{i, 0}}=\sum_{j=1}^{n} \overrightarrow{p_{i, 0} q_{i, j}^{\prime}} \overrightarrow{\underline{p_{i, 0} p_{i, j}}} \overrightarrow{p_{i, 0} p_{i, j}}+\overrightarrow{p_{0,0} p_{i, 0}},
$$

and the following equations hold:

$$
\begin{gathered}
\frac{\overrightarrow{p_{i, 0} q_{i, j}^{\prime}}}{\overrightarrow{p_{i, 0} p_{i, 1}}}=\frac{\overrightarrow{p_{0,0} q_{i, j}}}{\overrightarrow{p_{0,0} p_{0,1}}}, \quad 1 \leq j \leq n, \\
\frac{\tau_{p_{i}}-\tau_{p_{t_{O}}}}{\tau_{p_{t_{E}}}-\tau_{p_{t_{O}}}}=\frac{\overrightarrow{p_{0,0} q_{i, t}}}{\overline{p_{0,0} p_{0,1}}} .
\end{gathered}
$$

Using Equation (2), Equation (1) is equivalent to

$$
\overrightarrow{p_{0,0} p_{i}}=\sum_{j=1}^{n} \frac{\overrightarrow{p_{0,0} q_{i, j}}}{\overrightarrow{p_{0,0} p_{0,1}}} \overrightarrow{p_{i, 0} p_{i, j}}+\overrightarrow{p_{0,0} p_{i, 0}} .
$$

Considering the fact that $\alpha$ is a linear transformation, and using equation (4), the following holds:

$$
\alpha\left(\overrightarrow{p_{0,0} p_{i}}\right)=\sum_{j=1}^{n} \frac{\overrightarrow{p_{0,0} q_{i, j}}}{\overrightarrow{p_{0,0} p_{0,1}}} \alpha\left(\overrightarrow{p_{i, 0} p_{i, j}}\right)+\alpha\left(\overrightarrow{p_{0,0} p_{i, 0}}\right) .
$$

Moreover, let $e_{i}(\tau)$ be the $i$ th vector of the standard spatial basis at time $\tau$ and denote by $e_{i}=e_{i}(0)$. We then have

$$
\alpha\left(\overrightarrow{p_{0,0} p_{i}}\right)=\sum_{j=1}^{n} \frac{\overrightarrow{p_{0,0} q_{i, j}}}{\overline{p_{0,0} p_{0,1}}} \overrightarrow{e_{0}\left(\tau_{i}\right) e_{j}\left(\tau_{i}\right)}+\overrightarrow{e_{0} e_{0}\left(\tau_{i}\right)} .
$$

As equation (3) is invariant under elements of $\left(\mathcal{A}_{s t}^{f}, \mathcal{A}_{t}\right)$, we also have that

$$
\frac{\alpha\left(\tau_{p_{i}}\right)-\alpha\left(\tau_{p_{t_{O}}}\right)}{\alpha\left(\tau_{p_{t_{E}}}\right)-\alpha\left(\tau_{p_{t_{O}}}\right)}=\frac{\tau_{i}-0}{1-0}=\tau_{i}=\frac{\overrightarrow{p_{0,0} q_{i, t}}}{\overrightarrow{p_{0,0} p_{0,1}}} .
$$

So we have that:

$$
\alpha\left(\overrightarrow{p_{0,0} p_{i}}\right)=\sum_{j=1}^{n} \frac{\overrightarrow{p_{0,0} q_{i, j}}}{\overrightarrow{p_{0,0} p_{0,1}}} \overrightarrow{e_{0}\left(\tau_{i}\right) e_{j}\left(\tau_{i}\right)}+\frac{\overrightarrow{p_{0,0} q_{i, t}}}{\overline{p_{0,0} p_{0,1}}} \overrightarrow{e_{0} e_{n+1}} .
$$

ACM Journal Name, Vol. V, No. N, $20 Y Y$. 
Since all standard bases $\left(e_{0}\left(\tau_{i}\right), \ldots, e_{n}\left(\tau_{i}\right)\right)$ are parallel along the time axis, we have that

$$
\alpha\left(\overrightarrow{p_{0,0} p_{i}}\right)=\sum_{j=1}^{n} \frac{\overrightarrow{p_{0,0} q_{i, j}}}{\overrightarrow{p_{0,0} p_{0,1}}} \overrightarrow{e_{0} e_{j}}+\frac{\overrightarrow{p_{0,0} q_{i, t}}}{\overrightarrow{p_{0,0} p_{0,1}}} \overrightarrow{e_{0} e_{n+1}} .
$$

This completes the correctness proof for the conversion of relational predicates.

- The translation of composed formulas. When all the atomic subformulas of $\bar{\psi}$ have been translated as described above, the logical connectives can be added in a natural way. We assume that two atomic formulas $\bar{\chi}_{1}$ and $\bar{\chi}_{2}$ are translated already, into $\chi_{1}$ and $\chi_{2}$. The translations of $\bar{\chi}_{1} \wedge \bar{\chi}_{2}$ and $\bar{\chi}_{1} \vee \bar{\chi}_{2}$ are $\chi_{1} \wedge \chi_{2}$ and $\chi_{1} \vee \chi_{2}$, respectively. The formula $\neg \bar{\chi}_{1}$ is translated into $\neg \chi_{1}$.

Remember that with the conversion of a formula $\bar{\psi}_{\text {rel }}$ of type $\bar{R}\left(x_{1,1}, \ldots, x_{1, n}\right.$, $\left.x_{1, t}, \ldots, x_{m, 1}, \ldots, x_{m, n}, x_{m, t}\right)$ we assumed that $x_{i, t} \neq x_{j, t}$ for any $(1 \leq i<j<m)$. The reason is that we want to have only one affine coordinate system for every different time moment considered in that formula. Indeed, an element $\alpha$ of $\left(\mathcal{A}_{s t}^{f}, \mathcal{A}_{t}\right)$ is a one-to-one mapping from the snapshots of a certain input database $\mathbb{\$}$ to the snapshots of the output database $\alpha(\Phi)$. Therefore, we cannot map two different co-temporal coordinate systems to the same standard coordinate system using such an affinity.

Suppose now that $x_{i, t}=x_{j, t}$ for some $(1 \leq i<j \leq m)$. Then we adapt the previous translation with the extra requirement that $v_{i, k}=v_{j, k}$ for $k=0, \ldots, n$ and we have unique coordinate system for each point occurring in time.

When translating an $\mathrm{FO}(+, \times,<, 0,1, \bar{\sigma})$-formula $\bar{\psi}$, it is in general not known in advance which time coordinates are equal (this may depend on the input database; and it is undecidable in general which time coordinates are equal in an $\mathrm{FO}(+$, $\times,<, 0,1, \bar{\sigma}$ )-formula). To circumvent this problem, we consider all possible orders (using Before) of the time variables of $\bar{\psi}$ (a real variable denoting a time moment is recognized as it appears on the $i(n+1)$-th place $(i=1, \ldots, m)$ in the argument list of a spatio-temporal relation predicate) and take the disjunction over all possible orders of these time variables. We denote the set of all possible orders by $P$.

For each $\rho \in P$ the formula $\psi_{\rho}$ is the translation of $\bar{\psi}$ taken the (in)equalities into account according to the order of the time variables corresponding to $\rho$. Hence, each $\psi_{\rho}$ formula can have a different number $\ell_{\rho}$ of free variables, depending on $\rho$. We denote by $\ell$ the total number of free variables across all formulas $\psi_{\rho}, \rho \in P$.

When connecting several subformulas, the same principle has to be used, as arithmetic subformulas can impose equality on different time variables.

When applying the thus obtained translation of the quantifier-free part of $\psi$ to a spatio-temporal database instance, only some of the disjuncts will apply (possibly depending on the particular input database).

- Formulas with quantifiers. Finally, the quantifier prefix of $\bar{\psi}$ is translated in the natural way. Suppose that we already translated the quantifier-free formula $\bar{\chi}$ into the formula $\chi$. Then the translation of $(\exists x)(\bar{\chi})$ is $(\exists v)(\chi)$, where $v$ is the point variable associated to $x$ for which we have already declared Collinear $^{n}\left(u_{0,0}, u_{0,1}, v\right)$. 
Lemma 4.3. Let $\sigma$ be a spatio-temporal database schema. For every $\left(\mathcal{A}_{s t}^{f}, \mathcal{A}_{t}\right)$ generic spatio-temporal query expressible in $\mathrm{FO}(+, \times,<, 0,1, \bar{\sigma})$, there exists an equivalent $\mathrm{FO}\left(\left\{\right.\right.$ Between $^{n}$, Before, $\left.\left.\mathbf{E q C R}^{\text {st }}\right\}, \sigma\right)$-query.

Proof. Given a $\left(\mathcal{A}_{s t}^{f}, \mathcal{A}_{t}\right)$-generic spatio-temporal query of output type $(n, k)$, expressible in $\mathrm{FO}(+, \times,<, 0,1, \bar{\sigma})$,

$$
\bar{\psi}\left(x_{1,1}, \ldots, x_{1, n}, x_{1, t}, \ldots, x_{k, 1}, \ldots, x_{k, n}, x_{k, t}\right) .
$$

The conversion procedure, given in Lemma 4.2, returns a formula

$$
\begin{gathered}
\psi\left(u_{t_{O}}, u_{t_{E}}, u_{0,0}, \ldots, u_{0, n}, u_{1,0}, \ldots, u_{1, n}, \ldots, u_{k, 0}, \ldots, u_{k, n},\right. \\
\left.v_{1,1}, \ldots, v_{1, n}, v_{1, t}, \ldots, v_{k, 1}, \ldots, v_{k, n}, v_{k, t}\right),
\end{gathered}
$$

parameterized by one temporal and $k$ spatial coordinate systems and which is, up to a transformation of the group $\left(\mathcal{A}_{\underline{s t}}^{f}, \mathcal{A}_{t}\right)$, that depends on the coordinate systems, equivalent to the original formula $\bar{\psi}$. Since it has additional free variables, the query $\psi$ clearly has the wrong output type. $\mathrm{A} \mathrm{FO}(\Pi, \sigma)$-query equivalent to $\bar{\psi}$ should be a formula

$$
\psi_{\text {final }}\left(v_{1}, v_{2}, \ldots, v_{k}\right)
$$

having $k$ free variables only.

We obtain the desired formula by introducing $k$ new point variables $v_{i}$, and for each $1 \leq i \leq k, n$ new point variables $v_{i, 1}^{\prime}, \ldots, v_{i, n}^{\prime}$ such that $v_{i, j}^{\prime}$ is collinear with $u_{i, 0}$ and $u_{i, 1}$ and

$$
\operatorname{Coordinates}^{n}\left(u_{i, 0}, \ldots,, u_{i, n}, v_{i, 1}^{\prime}, \ldots, v_{i, n}^{\prime}, v_{i}\right) .
$$

Moreover, we require that

$$
\mathbf{E q C R}^{\mathbf{s t}}\left(u_{0,0}, u_{0,1}, v_{i, t}, u_{t_{O}}, u_{t_{E}}, v_{i}\right)
$$

and

$$
\bigwedge_{j=1}^{n} \mathbf{E q C R}^{\mathbf{s}}\left(u_{0,0}, u_{0,1}, v_{i, j}, u_{i, 0}, u_{i, 1}, v_{i, j}^{\prime}\right) .
$$

The final formula $\psi_{\text {final }}$ is now obtain by existentially quantifying all point variables, except for $v_{1}, \ldots, v_{k}$ in the conjunction of $\psi$ with the expressions (1), (2) and (3).

Now consider the (partial) output of $\psi_{\text {final }}$ when we choose a specific coordinate system for each set of variables $u_{i, 0}, \ldots, u_{i, n}$. By similar reasoning as in Lemma 4.2, we obtain that this partial output equals

$$
\alpha^{\prime-1}(\bar{\psi}(\alpha(\bar{\Phi})))
$$

where $\alpha^{\prime}=\left(\alpha_{s t}^{\prime}, \alpha_{t}^{\prime}\right)$ and $\alpha=\left(\alpha_{s t}, \alpha_{t}\right)$ both are transformations as specified in the statement of Lemma 4.2. This means that they both satisfy the same set of constraints, i.e., $\alpha_{t}^{\prime}=\alpha_{t}$ and for certain time moments $\tau, \alpha_{s t}^{\prime}(\tau)=\alpha_{s t}(\tau)$. In between those time moments $\alpha_{s t}^{\prime}$ and $\alpha_{s t}$ can differ. However, it follows from Lemma 4.2 that $\bar{\psi}(\alpha(\bar{\Phi}))=\bar{\psi}\left(\alpha^{\prime}(\overline{\mathscr{S}})\right)$, for any two transformations $\alpha$ and $\alpha^{\prime}$ satisfying the constraints as described in the statement of Lemma 4.2. Hence, we can conclude ACM Journal Name, Vol. V, No. N, $20 Y$ Y. 
without loss of generality that the partial output of $\psi_{\text {final }}$ when we fill in a specific coordinate system for each set of variables $u_{i, 0}, \ldots, u_{i, n}$ equals $\alpha^{-1}(\bar{\psi}(\alpha(\bar{\Phi}))$ ) where $\alpha$ is a transformation as specified in the statement of Lemma 4.2.

If we now consider all possible coordinate systems for each set of variables $u_{i, 0}, \ldots, u_{i, n}$

$$
\psi_{\text {final }}(\Phi)=\bigcup_{c} \bigcup_{\alpha_{c}}\left(\alpha_{c}^{-1}\left(\bar{\psi}\left(\alpha_{c}(\bar{\Phi})\right)\right),\right.
$$

where $c$ ranges over all possible coordinate system assignments and $\alpha_{c}$ ranges over all transformations satisfying the constraints following from this choice of coordinate systems.

The union $\bigcup_{c} \bigcup_{\alpha_{c}}\left(\alpha_{c}^{-1}\left(\bar{\psi}\left(\alpha_{c}(\bar{\Phi})\right)\right)\right)$ is in fact the union over all elements $\alpha$ of $\left(\mathcal{A}_{s t}^{f}, \mathcal{A}_{t}\right)$ of $\alpha^{-1}(\bar{\psi}(\alpha(\bar{\Phi})))$. So,

$$
\psi_{\text {final }}(\Phi)=\bigcup_{\alpha}\left(\alpha^{-1}(\bar{\psi}(\alpha(\bar{\Phi})))\right)
$$

where $\alpha$ ranges over all elements of $\left(\mathcal{A}_{s t}^{f}, \mathcal{A}_{t}\right)$.

Since $\psi_{\text {final }}$ is a $\left(\mathcal{A}_{s t}^{f}, \mathcal{A}_{t}\right)$-generic query and the group $\left(\mathcal{A}_{s t}^{f}, \mathcal{A}_{t}\right)$ is semi-algebraic (we give a precise definition in Section 5.3), we have that for every $\alpha \alpha^{-1}(\bar{\psi}(\alpha(\overline{\mathscr{S}})))=$ $\bar{\psi}(\bar{\Phi})$.

So, finally,

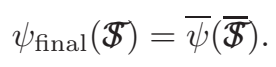

Proof of Theorem 4.6. Lemma 4.1, Lemma 4.2 and Lemma 4.3 together prove Theorem 4.6.

We are now ready to prove Proposition 4.1:

Proof of Proposition 4.1. Note that we only consider a finite number of moments in time in the proof of Lemma 4.2 (there are only a finite number of time variables in any $\mathrm{FO}(+, \times,<, 0,1, \bar{\sigma})$-formula $\varphi)$. This implies that the transformation groups $\mathcal{A}_{s t}^{f}$ and $\mathcal{A}_{s t}$ yield the same results. So, we can use the proof given above for the group $\left(\mathcal{A}_{s t}, \mathcal{A}_{t}\right)$. Indeed, in between the moments of time that are considered, it is indeed not important which transformation function is used.

Theorem 4.6 has a number of corollaries. We need two extra point predicates, namely EqDist ${ }^{\text {cotemp }}$ and Pos $^{n}$ before we can state those corollaries. First, EqDist ${ }^{\text {cotemp }}(p, q, r, s)$ is true for four co-temporal points $p, q, r$ and $s$ if and only if the (Euclidean) distance between $p$ and $q$ equals the distance between $r$ and $s$. Second, the expression $\operatorname{Pos}^{n}\left(p_{0}, p_{1}, \ldots, p_{n}\right)$ is true for $n+1$ co-temporal points $p_{0}, p_{1}, \ldots, p_{n}$ if and only if $\left(p_{0}, p_{1}, \ldots, p_{n}\right)$ forms a positively oriented coordinate system.

Corollary 4.1. Let $\sigma$ be a spatio-temporal database schema. Let $\left(\mathcal{F}_{s t}, \mathcal{F}_{t}\right)$ and $\left.\operatorname{FO}\left(\mathcal{F}_{s t}, \mathcal{F}_{t}\right)\right)$ be taken from Table III. The language $\mathcal{P}\left(\Pi\left(\mathcal{F}_{s t}, \mathcal{F}_{t}\right), \sigma\right)$ is sound and complete for the $\left(\mathcal{F}_{s t}, \mathcal{F}_{t}\right)$-generic spatio-temporal queries that are expressible in $\mathrm{FO}(+, \times,<, 0,1, \bar{\sigma})$. 


\begin{tabular}{|c|c|}
\hline$\left(\mathcal{F}_{s t}, \mathcal{F}_{t}\right)$ & Sets of point predicates $\Pi\left(\mathcal{F}_{s t}, \mathcal{F}_{t}\right)$ \\
\hline 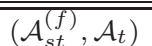 & 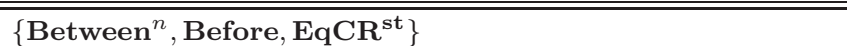 \\
\hline$\left(\mathcal{A}_{s t}^{(f)}, \mathcal{T}_{t}\right)$ & $\left\{\right.$ Between $^{n}$, Before, EqCR $^{\text {st }}$, UnitTime $\}$ \\
\hline$\left(\mathcal{A}_{s t}^{(f)}, I d_{t}\right)$ & $\left\{\right.$ Between $^{n}$, Before, EqCR $^{\text {st }}$, UnitTime, $\left.\mathbf{0}_{\mathbf{t}}, \mathbf{1}_{\mathbf{t}}\right\}$ \\
\hline$\left(\mathcal{S}_{s t}^{(f)}, \mathcal{F}_{t}\right)$ & $\Pi\left(\mathcal{A}_{s t}^{(f)}, \mathcal{F}_{t}\right) \cup\left\{\right.$ EqDist $^{\text {cotemp }\}}$ \\
\hline$\left(\mathcal{I}_{s t}^{(f)}, \mathcal{F}_{t}\right)$ & $\Pi\left(\mathcal{A}_{s t}^{(f)}, \mathcal{F}_{t}\right) \cup\left\{\right.$ EqDist ${ }^{\text {cotemp }}$, UnitDist $\}$ \\
\hline$\left(\mathcal{T}_{s t}^{(f)}, \mathcal{F}_{t}\right)$ & $\Pi\left(\mathcal{A}_{s t}^{(f)}, \mathcal{F}_{t}\right) \cup\left\{\right.$ EqDist ${ }^{\text {cotemp }}$, UnitDist $\left., \leq_{i}(1 \leq i \leq n), \mathbf{P o s}^{n}\right\}$ \\
\hline
\end{tabular}

Table III. An overview of the different sets of point predicate for some transformation groups. We have $\mathcal{F}_{t} \in\left\{\mathcal{A}_{t}, \mathcal{T}_{t}, I d_{t}\right\}$.

Proof. It follows directly from the proof of Theorem 4.6 that, for each subgroup $\left(\mathcal{F}_{s t}^{(f)}, \mathcal{F}_{t}\right)$ of $\left(\mathcal{A}_{s t}^{(f)}, \mathcal{A}_{t}\right)$, the language $\mathrm{FO}(\Pi, \sigma)$ is sound and complete for the $\left(\mathcal{F}_{s t}^{(f)}, \mathcal{F}_{t}\right)$-generic queries expressible in $\mathrm{FO}(+, \times,<, 0,1, \bar{\sigma})$ if and only if the following three conditions are satisfied:

(i) the set $\Pi$ contains the elements Between ${ }^{n}$, Before and $\mathbf{E q C R}{ }^{\text {st }}$;

(ii) all elements of $\Pi$ are $\mathrm{FO}(+, \times,<, 0,1, \bar{\sigma})$-expressible and invariant under the transformations of $\left(\mathcal{F}_{s t}^{(f)}, \mathcal{F}_{t}\right)$;

(iii) the facts " $\left(v_{0}, v_{1}, \ldots, v_{n}\right)$ is the image of the standard coordinate system in the hyperplane co-temporal with $v_{t_{O}}$ under an element of $\left(\mathcal{F}_{s t}^{(f)}, \mathcal{F}_{t}\right)$ " and " $\left(v_{t_{O}}, v_{t_{E}}\right)$ is the image of the standard temporal coordinate system under an element of $\left(\mathcal{F}_{s t}^{(f)}, \mathcal{F}_{t}\right)$ ", where $v_{0}, v_{1}, \ldots, v_{n}, v_{t_{O}}$ and $v_{t_{E}}$ are points in $(n+1)$ dimensional real space, are expressible in $\mathrm{FO}(\Pi)$.

All groups listed in Table III are subgroups of $\left(\mathcal{A}_{s t}^{(f)}, \mathcal{A}_{t}\right)$ and satisfy the first condition. It is also straightforward to verify that they satisfy the second condition.

For the third condition, we list for every group mentioned in Table III the expressions for the spatial and temporal coordinate system. The proof that these expressions are correct are straightforward.

- For the group $\left(\mathcal{A}_{\text {st }}^{(f)}, \mathcal{A}_{t}\right)$, the expressions for $\operatorname{TCoSys}_{\mathcal{A}}\left(u_{1}, u_{2}\right)$ and $\operatorname{CoSys}_{\mathcal{A}}^{n}($ $\left.u_{0}, u_{1}, \ldots, u_{n}\right)$ were given in Lemma 4.2 .

- For the group $\left(\mathcal{A}_{s t}^{(f)}, \mathcal{I}_{t}\right)$, the expression for the spatial coordinate system does not change, but

$$
\operatorname{TCoSys}_{\mathcal{T}}\left(u_{1}, u_{2}\right):=\operatorname{TCoSys}_{\mathcal{A}}\left(u_{1}, u_{2}\right) \wedge \operatorname{UnitTime}\left(u_{1}, u_{2}\right) .
$$

- For the group $\left(\mathcal{A}_{s t}^{(f)}, I d_{t}\right)$, the expression for the spatial coordinate system does again not change, but

$$
\operatorname{TCoSys}_{I d}\left(u_{1}, u_{2}\right):=\operatorname{TCoSys}_{\mathcal{T}}\left(u_{1}, u_{2}\right) \wedge \mathbf{0}_{\mathbf{t}}\left(u_{1}\right) \wedge \mathbf{1}_{\mathbf{t}}\left(u_{2}\right) .
$$

For the following groups, we only list the expression for the spatial coordinate system. The temporal coordinate system depends on the groups $\mathcal{F}_{t}$ and is completely analogous to the previous cases.

ACM Journal Name, Vol. V, No. N, 20YY. 
- For the group $\left(\mathcal{S}_{s t}^{(f)}, \mathcal{F}_{t}\right)$, we have

$\operatorname{CoSys}_{\mathcal{S}}\left(u_{0}, u_{1}, \ldots, u_{n}\right):=$

$$
\operatorname{CoSys}_{\mathcal{A}}^{n}\left(u_{0}, u_{1}, \ldots, u_{n}\right) \wedge \bigwedge_{i=1}^{n} \bigwedge_{j=1}^{n} \operatorname{EqDist}^{\operatorname{cotemp}}\left(u_{0}, u_{i}, u_{0}, u_{j}\right)
$$

- For the group $\left(\mathcal{I}_{s t}^{(f)}, \mathcal{F}_{t}\right)$, we have

$$
\operatorname{CoSys}_{\mathcal{I}}\left(u_{0}, u_{1}, \ldots, u_{n}\right):=\operatorname{CoSys}_{\mathcal{S}}\left(u_{0}, u_{1}, \ldots, u_{n}\right) \wedge \bigwedge_{i=1}^{n} \operatorname{UnitDist}\left(u_{0}, u_{i}\right) .
$$

- For the group $\left(\mathcal{T}_{s t}^{(f)}, \mathcal{F}_{t}\right)$, we have

$\operatorname{CoSys}_{\mathcal{T}}\left(u_{0}, u_{1}, \ldots, u_{n}\right):=$

$$
\operatorname{CoSys}_{\mathcal{I}}\left(u_{0}, u_{1}, \ldots, u_{n}\right) \wedge \bigwedge_{i=1}^{n} \bigwedge_{j=1}^{n} \leq_{i}\left(u_{0}, u_{j}\right) \wedge \operatorname{Pos}^{n}\left(u_{0}, u_{1}, \ldots, u_{n}\right) .
$$

Next, we illustrate the languages summarized in Table III with the appropriate examples of Section 3.5.

Example 4.3. We give the $\mathrm{FO}\left(\left\{\right.\right.$ Between $^{n}$, Before $\left.\left.\mathbf{E q C R}^{\text {st }}\right\}\right)$-expression $\varphi_{3}^{\prime}$ equivalent to the $\left(\mathcal{I}_{s t}, \mathcal{A}_{t}\right)$-generic query of Example 3.3: Was there a collision between car $A$ and car $B$ ?:

$$
\varphi_{3}^{\prime}:=(\exists u)(\operatorname{car} A(u) \wedge \operatorname{car} B(u)) .
$$

Remark that this query can be expressed without the use of the point predicates from $\Pi$.

ExAmple 4.4. We give the $\mathrm{FO}\left(\left\{\right.\right.$ Between $^{n}$, Before $\mathbf{E q C R}^{\text {st }}, \mathbf{E q D i s t}^{\text {cotemp }}$, UnitDist, $\leq_{i}(1 \leq i \leq n), \mathbf{P o s}^{n}$, UnitTime, $\left.\left.\mathbf{0}_{\mathbf{t}}, \mathbf{1}_{\mathbf{t}}\right\}\right)$ expression $\varphi_{4}^{\prime}$ equivalent to the $\left(\mathcal{T}_{s t}, i d_{t}\right)$-generic query of Example 3.4: Did car A pass at 500 meters north of car $B$ at time moment $t=5930$ ?

The fact that a point has time coordinate 5930 can be expressed using UnitTime, $\mathbf{0}_{\mathbf{t}}$, and $\mathbf{1}_{\mathbf{t}}$. We illustrate this with a predicate expressing the fact that a point has time coordinate 3 :

$$
\begin{aligned}
e q 3 t(u):=\left(\exists v_{1}\right)\left(\exists v_{2}\right)\left(\mathbf{1}_{\mathbf{t}}\left(v_{1}\right) \wedge \operatorname{Before}\left(v_{1}, v_{2}\right)\right. & \wedge \operatorname{UnitTime}\left(v_{1}, v_{2}\right) \wedge \\
\operatorname{Before}\left(v_{2}, u\right) & \left.\wedge \operatorname{UnitTime}\left(v_{2}, u\right)\right) .
\end{aligned}
$$

The fact that the distance between two points is 500 can be expressed using UnitDist in a way comparable to the construction of the predicate $3 \mathrm{sec}$ of Example 4.1.

Now we give the expression $\varphi_{4}^{\prime}$ :

$$
\begin{aligned}
& (\exists u)(\exists v)(\exists w)(\operatorname{car} A(u) \wedge \operatorname{car} B(v) \wedge \operatorname{eq5930t}(u) \wedge \operatorname{eq5930t}(v) \wedge \\
& \left.\left(\leq_{1}(u, w) \wedge \leq_{1}(w, u)\right) \wedge\left(\leq_{2}(v, w) \wedge \leq_{2}(w, v)\right) \wedge 500 \text { meters }(u, w)\right) .
\end{aligned}
$$




\section{SOUND AND COMPLETE LANGUAGES FOR THE COMPUTABLE GENERIC SPATIO-TEMPORAL QUERIES}

In this section, we show that the languages $\operatorname{FO}\left(\Pi\left(\mathcal{F}_{s t}, \mathcal{F}_{t}\right), \sigma\right)$ of the previous section, when extended with assignment statements and a While loop, yield languages that are computationally sound and complete for the computable queries that are $\left(\mathcal{F}_{s t}, \mathcal{F}_{t}\right)$-generic. To start with, we explain in more detail how point-based logics are extended with assignment statements and a while loop. Afterwards, this section is organized in the same way as Section 4. We first discuss sound and complete languages for the queries generic for time-independent transformation groups. Then we focus on genericity for groups related to physical notions. Finally, we address sound and complete languages for the queries that are generic for the time-dependent transformations.

We start with extending the point-based logics described in Definition 4.1 with while loops.

Definition 5.1. Let $\Pi$ be a finite set of point predicates, and let $\sigma$ be a database schema. Syntactically, a program in the language $\mathrm{FO}(\Pi, \sigma)+$ While is a finite sequence of statements and while-loops. It is assumed there is a sufficient supply of new relation variables, each with an appropriate arity.

(i) Each statement has the form

$$
R:=\left\{\left(u_{1}, \ldots, u_{k}\right) \mid \varphi\left(u_{1}, \ldots, u_{k}\right)\right\} ; .
$$

Here, $R$ is a new relation variable with assigned arity $k$ (the variables $u_{i}$ range over $\left.\mathbf{R}^{n} \times \mathbf{R}\right)$ and $\varphi$ is a formula in $\operatorname{FO}\left(\Pi, \sigma^{\prime}\right)$, where $\sigma^{\prime}$ is the set of relation names containing the elements of $\sigma$ together with the relation variables introduced in previous statements of the program.

(ii) A while-loop has the form

$$
\text { while } \varphi \text { do } P \text {; }
$$

where $P$ is a program and $\varphi$ is a sentence in $\operatorname{FO}\left(\Pi, \sigma^{\prime}\right)$, where $\sigma^{\prime}$ is again the set of relation names containing the elements of $\sigma$ together with the relation variables introduced in previous statements of the program.

(iii) One of the relation names occurring in the program is designated as the output relation and is named $R_{\text {out }}$.

Semantically, a program in the query language $\mathrm{FO}(\Pi, \sigma)+$ While expresses a spatiotemporal query as soon as $R_{\text {out }}$ is assigned a return value. The execution of a $\mathrm{FO}(\Pi, \sigma)+$ While-program applied to an input database is performed step-by-step. A statement is executed by first evaluating the $\mathrm{FO}(\Pi, \sigma)$-formula on the right hand side on the input database together with the newly created relations resulting from previous statements. Next, the result of the evaluation of the right hand side is assigned to the relation variable on the left-hand side. The effect of a while loop is to execute the body as long as the condition $\varphi$ evaluates to true.

Note that a $\mathrm{FO}(\Pi, \sigma)+$ While-program is not guaranteed to halt. For those input databases it does not, the query represented by the program is not defined on that particular input database.

ACM Journal Name, Vol. V, No. N, 20YY. 
Consider the following example which will be used later on to express the query from Example 3.1.

EXAMPLE 5.1. Suppose that we have a spatio-temporal database with schema $\sigma=\{R, S\}$, where the underlying dimension is two and both $R$ and $S$ have arity one. We assume that all points in $R$ and $S$ have disjoint time coordinates. This means that we can sort all points according to their time coordinates. We also assume that $R$ and $S$ both contain a finite number of points.

The query $Q$ we want to answer is the following: Does $R$ contain more points than $S$ ?. It is well known that we cannot express this query in first-order logic [Grumbach and $\mathrm{Su}$ 1997].

The $\mathrm{FO}(\Pi, \sigma)+$ While-program expressing $Q$ is:

$$
\begin{gathered}
R_{\text {Not }}:=\{\} ; \\
S_{\text {Not }}:=\{\} ; \\
R_{\text {Smallest }}:=\left\{(u) \mid R(u) \wedge \neg R_{\text {Not }}(u) \wedge\right. \\
\left.\quad(\forall v)\left(\left(R(v) \wedge \neg R_{\text {Not }}(v)\right) \rightarrow(\text { Before }(u, v))\right)\right\} ; \\
S_{\text {Smallest }}:=\left\{(u) \mid S(u) \wedge \neg S_{\text {Not }}(u) \wedge\right. \\
\left.\quad(\forall v)\left(\left(S(v) \wedge \neg S_{\text {Not }}(v)\right) \rightarrow(\text { Before }(u, v))\right)\right\} ; \\
\text { While }(\exists u)\left(R_{\text {Smallest }}(u)\right) \wedge(\exists v)\left(S_{\text {Smallest }}(v)\right) \text { do } \\
R_{\text {Not }}:=\left\{(u) \mid R_{\text {Not }}(u) \vee R_{\text {Smallest }}(u)\right\} ; \\
S_{\text {Not }}:=\left\{(u) \mid S_{\text {Not }}(u) \vee S_{\text {Smallest }}(u)\right\} ; \\
R_{\text {Smallest }}:=\left\{(u) \mid R(u) \wedge \neg R_{\text {Not }}(u) \wedge\right. \\
\left.\quad(\forall v)\left(\left(R(v) \wedge \neg R_{\text {Not }}(v)\right) \rightarrow(\text { Before }(u, v))\right)\right\} ; \\
S_{\text {Smallest }}:=\left\{(u) \mid S(u) \wedge \neg S_{\text {Not }}(u) \wedge\right. \\
\left.\quad(\forall v)\left(\left(S(v) \wedge \neg S_{\text {Not }}(v)\right) \rightarrow(\text { Before }(u, v))\right)\right\} ; \\
R_{\text {out }}:=\left\{() \mid(\exists u)\left(R_{\text {Smallest }}(u)\right)\right\} ;
\end{gathered}
$$

Intuitively, this program repeatedly takes the earliest point from both $R$ and $S$ until they do not both contain unvisited points anymore. When the while loop terminates and $R$ still contains unvisited points, true is returned.

\subsection{Genericity for time-independent transformations}

In this section, we prove a general result concerning computable $\left(\mathcal{F}_{s t}, \mathcal{F}_{t}\right)$-generic queries where $\left(\mathcal{F}_{s t}, \mathcal{F}_{t}\right)$ is a time-independent affinity of $\mathbf{R}^{n} \times \mathbf{R}$, i.e., a group from Table I.

The following theorem follows directly from the proof of Theorem 6.1 [Gyssens et al. 1999].

Theorem 5.1. Let $\sigma$ be a spatio-temporal database schema. Let $\mathcal{F}$ be a subgroup of the affinities of $\mathbf{R}^{n} \times \mathbf{R}$, let $\Pi$ be a set of point predicates and let $\mathrm{FO}(\Pi, \sigma)$ be a point language that is sound and complete for the $\mathcal{F}$-generic queries expressible in $\mathrm{FO}(+, \times,<, 0,1, \bar{\sigma})$. Then the language $\mathrm{FO}(\Pi)+$ While is sound and complete for the $\mathcal{F}$-generic computable queries.

From this, we can derive the following result:

Corollary 5.1. Let $\sigma$ be a spatio-temporal database schema. Let $\left(\mathcal{F}_{s t}, \mathcal{F}_{t}\right)$ be a group and let $\Pi\left(\mathcal{F}_{s t}, \mathcal{F}_{t}\right)$ be as in Table I. The point language $\mathrm{FO}\left(\Pi\left(\mathcal{F}_{s t}, \mathcal{F}_{t}\right), \sigma\right)$ + While is sound and complete for the computable $\left(\mathcal{F}_{s t}, \mathcal{F}_{t}\right)$-generic queries over $\bar{\sigma}$. 
Proof. The correctness follows from Theorem 4.4 and Theorem 5.1.

\subsection{Applications to Physics}

Here, we focus again on the transformation groups $\left(\mathcal{V}_{s t}, \mathcal{T}_{t}\right),\left(\mathcal{V}(\mathcal{R})_{s t}, \mathcal{T}_{t}\right),\left(\mathcal{A C}_{s t}\right.$, $\left.\mathcal{T}_{t}\right)$ and $\left(\mathcal{A C}(\mathcal{R})_{s t}, \mathcal{T}_{t}\right)$. As they are all subgroups of the affinities of $\mathbf{R}^{n} \times \mathbf{R}$, we can apply Theorem 5.1 again.

Corollary 5.2. Let $\sigma$ be a spatio-temporal database schema. Let $\left(\mathcal{F}_{s t}, \mathcal{T}_{t}\right)$ be a group from Table II and let $\Pi\left(\mathcal{F}_{s t}, \mathcal{T}_{t}\right)$ be as in Table II. The point language $\mathrm{FO}\left(\Pi\left(\mathcal{F}_{s t}, \mathcal{T}_{t}\right), \sigma\right)+$ While is sound and complete for the computable spatio-temporal queries over $\bar{\sigma}$ that are $\left(\mathcal{F}_{s t}, \mathcal{I}_{t}\right)$-generic.

Proof. The correctness follows from Theorem 4.5 and Theorem 5.1.

Example 5.2. We now give the $\mathrm{FO}\left(\left\{\right.\right.$ Between $^{n+1}$, Before, $\left.=_{\text {space }}\right)+$ Whileprogram expressing query $Q_{1}$ of Example 3.1: Does the route followed by car $A$ self-intersect more often than the route followed by car $B$ does?.

If a car is standing still at a certain position, this will result in an infinite number of points in $\mathbf{R}^{n} \times \mathbf{R}$ with the same spatial coordinates. However, one would not consider this situation to be an infinite number of self-intersections. Therefore, when such a situation happens, we only consider the last moment of the interval during which the car is at that specific location.

Intuitively, the program first computes the relations containing all self-intersections of the trajectories of both cars, and then determines whether the route of car A self-intersects the most. The program of Example 5.1 can be used to perform this last task. We slightly adapt it such that it expresses query $Q_{1}$ :

$$
\begin{aligned}
& A_{\cap}:=\left\{(u) \mid \operatorname{car} A(u) \wedge(\exists v)\left(\operatorname{car} A(v) \wedge \operatorname{Before}(u, v) \wedge=_{\text {space }}(u, v) \wedge\right.\right. \\
& (\forall w)((\operatorname{car} A(w) \wedge \operatorname{Before}(u, w) \wedge \operatorname{Before}(w, v) \wedge u \neq w \wedge v \neq w) \\
& \left.\left.\left.\rightarrow \neg\left(=_{\text {space }}(w, v)\right)\right)\right)\right\} \text {; } \\
& B_{\cap}:=\left\{(u) \mid \operatorname{car} B(u) \wedge(\exists v)\left(\operatorname{car} B(v) \wedge \operatorname{Before}(u, v) \wedge=_{\text {space }}(u, v) \wedge\right.\right. \\
& (\forall w)((\operatorname{car} B(w) \wedge \operatorname{Before}(u, w) \wedge \operatorname{Before}(w, v) \wedge u \neq w \wedge v \neq w) \\
& \left.\left.\left.\rightarrow \neg\left(=_{\text {space }}(w, v)\right)\right)\right)\right\} \text {; } \\
& A_{\mathrm{Not}}:=\{\} \\
& B_{\mathrm{Not}}:=\{\} \\
& A_{\text {Smallest }}:=\left\{(u) \mid A_{\cap}(u) \wedge \neg A_{\text {Not }}(u) \wedge(\forall v)(\right. \\
& \left.\left.\left(A_{\cap}(v) \wedge \neg A_{\mathrm{Not}}(v)\right) \rightarrow(\operatorname{Before}(u, v))\right)\right\} ; \\
& B_{\text {Smallest }}:=\left\{(u) \mid B_{\cap}(u) \wedge \neg B_{\text {Not }}(u) \wedge(\forall v)(\right. \\
& \left.\left.\left(B_{\cap}(v) \wedge \neg B_{\text {Not }}(v)\right) \rightarrow(\text { Before }(u, v))\right)\right\} ; \\
& \text { While }(\exists u)\left(A_{\text {Smallest }}(u)\right) \wedge(\exists v)\left(B_{\text {Smallest }}(v)\right) \text { do } \\
& A_{\text {Not }}:=\left\{(u) \mid A_{\text {Not }}(u) \vee A_{\text {Smallest }}(u)\right\} \\
& B_{\text {Not }}:=\left\{(u) \mid B_{\text {Not }}(u) \vee B_{\text {Smallest }}(u)\right\} ; \\
& A_{\text {Smallest }}:=\left\{(u) \mid A_{\cap}(u) \wedge \neg A_{\text {Not }}(u) \wedge(\forall v)(\right. \\
& \left.\left.\left(A_{\cap}(v) \wedge \neg A_{\text {Not }}(v)\right) \rightarrow(\text { Before }(u, v))\right)\right\} ; \\
& B_{\text {Smallest }}:=\left\{(u) \mid B_{\cap}(u) \wedge \neg B_{\text {Not }}(u) \wedge(\forall v)(\right. \\
& \left.\left.\left(B(v) \wedge \neg B_{\mathrm{Not}}(v)\right) \rightarrow(\operatorname{Before}(u, v))\right)\right\} ; \\
& R_{\text {out }}:=\left\{() \mid(\exists u)\left(A_{\text {Smallest }}(u)\right)\right\} ;
\end{aligned}
$$




\subsection{Genericity for time-dependent transformations}

Finally, we study notions of genericity determined by groups of time-dependent transformations. Here, we only show results for the groups of arbitrary timedependent transformations $\mathcal{F}_{s t}$. We concentrate on the group $\left(\mathcal{A}_{s t}, \mathcal{A}_{t}\right)$. The other time-dependent transformation groups will be addressed afterwards (Corollary 5.3). For the groups $\mathcal{F}_{s t}^{f}$ the problem of identifying sound and complete languages is open, we will discuss the problems concerning this at the end of this section.

We introduce some definitions first. Recall that we introduced, in Section 3.1, the abbreviation $f\left(R^{\Phi}\right)$ for the formula $\left\{\left(f\left(\mathbf{a}_{1}, \tau_{1}\right), f\left(\mathbf{a}_{2}, \tau_{2}\right), \ldots, f\left(\mathbf{a}_{k}, \tau_{k}\right)\right) \mid\left(\mathbf{a}_{1}, \tau_{1}\right.\right.$, $\left.\left.\mathbf{a}_{2}, \tau_{2}, \ldots, \mathbf{a}_{k}, \tau_{k}\right) \in R^{\mathbb{S}}\right\}$, where $R$ is a relation name and $\mathbb{\$}$ a spatio-temporal database over a schema $\sigma$ that contains $R$.

Definition 5.2. Let $\mathbb{S}_{1}$ and $\mathbb{S}_{2}$ be spatio-temporal databases over the schema $\sigma=\left\{R_{1}, \ldots, R_{m}\right\}$ with underlying dimension $n$. The databases $\mathbb{S}_{1}$ and $\mathbb{S}_{2}$ are called $\left(\mathcal{F}_{s t}, \mathcal{F}_{t}\right)$-isomorphic if and only if there exists a $f=\left(f_{s t}, f_{t}\right) \in\left(\mathcal{F}_{s t}, \mathcal{F}_{t}\right)$ such that for all $R_{i}$ in $\sigma, f\left(R_{i}^{\Phi_{1}}\right)=R_{i}^{\mathbb{S}_{2}}$.

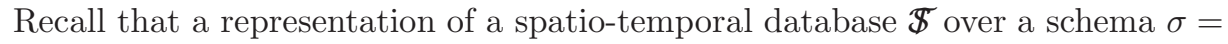
$\left\{R_{1}, \ldots, R_{m}\right\}$ is a tuple $\left(\varphi_{1}, \ldots, \varphi_{m}\right)$ of quantifier-free formulas in $\mathrm{FO}(+, \times,<, 0$, $1)$, such that $\varphi_{i}$ describes $R_{i}^{\mathbb{S}}$.

Assuming some order on the characters or symbols that may appear in a $\mathrm{FO}(+$, $\times,<, 0,1)$-formulas, we can lexicographically order the $\mathrm{FO}(+, \times,<, 0,1)$-formulas.

Definition 5.3. The $\left(\mathcal{F}_{s t}, \mathcal{F}_{t}\right)$-canonization of a spatio-temporal database $\mathbb{\$}$ over a schema $\sigma=\left\{R_{1}, \ldots, R_{m}\right\}$, denoted by $\operatorname{Canon}_{\left(\mathcal{F}_{s t}, \mathcal{F}_{t}\right)}(\mathbb{S})$, is the spatiotemporal database $\mathbb{S}^{\prime}$, which is $\left(\mathcal{F}_{s t}, \mathcal{F}_{t}\right)$-isomorphic to $\mathbb{S}$ and has a representation by quantifier-free $\mathrm{FO}(+, \times,<, 0,1)$-formulas

$$
\left(\varphi_{\mathbf{C a n o n}_{\left(\mathcal{F}_{s t}, \mathcal{F}_{t}\right)}\left(R_{1}\right)}, \ldots, \varphi_{\mathbf{C a n o n}_{\left(\mathcal{F}_{s t}, \mathcal{F}_{t}\right)}\left(R_{1}\right)}\right)
$$

that occurs lexicographically first among the representations of spatio-temporal databases $\left(\mathcal{F}_{s t}, \mathcal{F}_{t}\right)$-isomorphic to $\mathbb{\$}$.

Definition 5.4. Let $\mathbb{S}$ be a spatio-temporal database. The $\left(\mathcal{F}_{\text {st }}, \mathcal{F}_{t}\right)$-type of $\mathbb{S}$, denoted $\operatorname{Type}_{\left(\mathcal{F}_{s t}, \mathcal{F}_{t}\right)}(\mathbb{\Phi})$, equals

$$
\left\{f \in\left(\mathcal{F}_{s t}, \mathcal{F}_{t}\right) \mid f(\mathbb{\Phi})=\operatorname{Canon}_{\left(\mathcal{F}_{s t}, \mathcal{F}_{t}\right)}(\mathbb{\Phi})\right\}
$$

We can derive directly from a similar proposition of Gyssens, Van den Bussche and Van Gucht [Gyssens et al. 1999] that, for a spatio-temporal database $\mathbb{\$}$, a representation of $\operatorname{Canon}_{\left(\mathcal{F}_{s t}, \mathcal{F}_{t}\right)}(\mathbb{S})$ can be computed if and only if $\left(\mathcal{F}_{s t}, \mathcal{F}_{t}\right)$ is a transformation group.

A transformation group $\mathcal{G}$ of $\mathbf{R}^{n} \times \mathbf{R}$ is semi-algebraic if and only if there exists a semi-algebraic subset of $\mathbf{R}^{l}$, described by a $\mathrm{FO}(+, \times,<, 0,1)$-formula $\varphi_{\mathcal{G}}$, for some fixed $l$, representing all elements of $\mathcal{G}$, such that the set

$$
\begin{aligned}
\left\{\left(g_{1}, \ldots, g_{l}, x_{1}, \ldots, x_{n}, t, x_{1}^{\prime}, \ldots, x_{n}^{\prime}, t^{\prime}\right) \mid\right. & \\
\varphi_{\mathcal{G}}\left(g_{1}, \ldots, g_{l}\right) & \left.\wedge \varphi_{\mathcal{G}-i m g}\left(g_{1}, \ldots, g_{l}, x_{1}, \ldots, x_{n}, t, x_{1}^{\prime}, \ldots, x_{n}^{\prime}, t^{\prime}\right)\right\}
\end{aligned}
$$


also called the graph of $\mathcal{G}$, is a semi algebraic subset of $\mathbf{R}^{l+2(n+1)}$. The formula $\varphi_{\mathcal{G}-i m g}$ expresses that, for the element of $\mathcal{G}$ represented by the tuple $\left(g_{1}, \ldots, g_{l}\right)$, the tuple $\left(x_{1}, \ldots, x_{n}, t\right)$ is mapped to $\left(x_{1}^{\prime}, \ldots, x_{n}^{\prime}, t^{\prime}\right)$.

REMARK 5.1. The transformation group $\left(\mathcal{A}_{s t}, \mathcal{A}_{t}\right)$ is semi-algebraic if and only if the transformation groups $\mathcal{A}_{s t}$ and $\mathcal{A}_{t}$ are both semi-algebraic. The semialgebraic set for the group $\mathcal{A}_{t}$ is $\varphi_{\mathcal{A}_{t}} \equiv\left\{(\alpha, \beta) \in \mathbf{R}^{2} \mid \alpha>0\right\}$. The semialgebraic set associated to the group $\mathcal{A}_{s t}$ is given by the $\mathrm{FO}(+, \times,<, 0,1)$-formula $\varphi_{\mathcal{A}_{s t}}\left(\alpha_{1,1}, \ldots, \alpha_{1, n}, \ldots, \alpha_{n, 1}, \ldots, \alpha_{n, n}, \beta_{1}, \ldots, \beta_{n}, t\right)$ expressed by

$$
\left|\begin{array}{ccc}
\alpha_{1,1} & \cdots & \alpha_{1, n} \\
\vdots & \cdots & \vdots \\
\alpha_{n, 1} & \cdots & \alpha_{n, n}
\end{array}\right| \neq 0
$$

We now give the formulas for $\varphi_{\mathcal{A}_{t}-i m g}$ and $\varphi_{\mathcal{A}_{s t}-i m g}$. The formula $\varphi_{\mathcal{A}_{t}-i m g}(\alpha$, $\left.\beta, x_{1}, \ldots, x_{n}, x_{t}, x_{1}^{\prime}, \ldots, x_{n}^{\prime}, x_{t}^{\prime}\right)$ can be given as

$$
x_{t}^{\prime}=\alpha x_{t}+\beta \wedge \bigwedge_{i=1}^{n} x_{i}^{\prime}=x_{i} .
$$

The formula $\varphi_{\mathcal{A}_{s t}-i m g}\left(\alpha_{1,1}, \ldots, \alpha_{1, n}, \ldots, \alpha_{n, 1}, \ldots, \alpha_{n, n}, \beta_{1}, \ldots, \beta_{n}, t, x_{1}, \ldots, x_{n}, x_{t}\right.$, $\left.x_{1}^{\prime}, \ldots, x_{n}^{\prime}, x_{t}^{\prime}\right)$ on the other hand is

$$
t=t_{x} \wedge t_{x}=t_{x}^{\prime} \wedge \bigwedge_{i=1}^{n} \alpha_{i, 1} x_{1}+\cdots+\alpha_{i, n} x_{n}+\beta_{i}=x_{i}^{\prime} .
$$

The graph of the group $\left(\mathcal{A}_{s t}, \mathcal{A}_{t}\right)$ is now a semi-algebraic subset of $\mathbf{R}^{l+2(n+1)}$, where $l=n^{2}+n+3$, described as follows:

$$
\begin{aligned}
& \gamma_{\left(\mathcal{A}_{s t}, \mathcal{A}_{t}\right)}\left(\alpha_{1,1}, \ldots, \alpha_{1, n}, \ldots,\right. \alpha_{n, 1}, \ldots, \alpha_{n, n}, \beta_{1}, \ldots, \beta_{n}, t \\
&\left.\alpha, \beta, x_{1}, \ldots, x_{n}, t_{x}, x_{1}^{\prime}, \ldots, x_{n}^{\prime}, t_{x}^{\prime}\right):= \\
& \varphi_{\mathcal{A}_{s t}-i m g}\left(\alpha_{1,1}, \ldots, \alpha_{1, n}, \ldots, \alpha_{n, 1}, \ldots, \alpha_{n, n}, \beta_{1}, \ldots, \beta_{n}, t\right. \\
&\left.x_{1}, \ldots, x_{n}, x_{t}, x_{1}^{\prime}, \ldots, x_{n}^{\prime}, x_{t}\right) \\
& \quad \wedge \varphi_{\mathcal{A}_{t}-i m g}\left(\alpha, \beta, x_{1}^{\prime}, \ldots, x_{n}^{\prime}, x_{t}, x_{1}^{\prime}, \ldots, x_{n}^{\prime}, x_{t}^{\prime}\right) .
\end{aligned}
$$

We now prove the main theorem of this section. The proof technique used here was introduced by Gyssens, Van den Bussche and Van Gucht [Gyssens et al. 1999]. We first sketch the proof technique, but only give details about the aspects of the proof that need modifications in the context of spatio-temporal databases. These modifications are based on proof techniques introduced in Section 4.

Theorem 5.2. Let $\sigma$ be a spatio-temporal database schema. The point language $\mathrm{FO}\left(\left\{\right.\right.$ Between $^{n}$, Before, $\left.\left.\mathbf{E q C R}^{\text {st }}\right\}, \sigma\right)+$ While is sound and complete for the $\left(\mathcal{A}_{s t}\right.$, $\mathcal{A}_{t}$ )-generic computable spatio-temporal queries over $\bar{\sigma}$.

ACM Journal Name, Vol. V, No. N, $20 Y$ Y. 
Proof. It suffices to show that an $\left(\mathcal{A}_{s t}, \mathcal{A}_{t}\right)$-generic computable query $Q$ over $\bar{\sigma}$ can be simulated in the language $\mathrm{FO}\left(\left\{\right.\right.$ Between $^{n}$, Before, $\left.\left.\mathbf{E q C R} \mathbf{C R}^{\text {st }}\right\}, \sigma\right)+$ While. We first briefly sketch the proof strategy, including the conversion procedure and the encoding and decoding step, that appear in it. Later the coding and decoding will be explained in more detail. For the remainder of this proof, $\Pi$ will denote the set $\left\{\right.$ Between $^{n}$, Before, $\left.\mathbf{E q C R}^{\text {st }}\right\}$.

We start with the encoding that will be used to convert formulas that represent spatio-temporal relations into natural numbers

- The encoding mechanism. Let $\$$ be a spatio-temporal database over $\sigma$. Let $K$ be the maximum of the arities of all relations in $\sigma$ and the query $Q$. Let $n$ be the underlying dimension. Then each relation of $\$$ can be represented by a quantifierfree $\mathrm{FO}(+, \times,<, 0,1)$-formula using only the variables $x_{1}, \ldots, x_{(n+1) K}$, the symbols $\leq,+, \times,(),, \vee$ and $\neg$, and the constants 0 and 1 .

We denote these $9+(n+1) K$ by $s_{1}, \ldots, s_{9+(n+1) K}$. Hence, we can encode a quantifier-free $\mathrm{FO}(+, \times,<, 0,1)$-formula as a string $s=s_{i_{1}} \ldots s_{i_{k}}$ as the natural number $N=p_{1}^{i_{1}} \ldots p_{k}^{i_{k}}$, where $p_{j}$ is the $j$-th prime number. And we denote $N$ by $\operatorname{Encode}(s)$.

- Proof strategy. Given a spatio-temporal database $\$$ over a schema $\sigma=\left\{R_{1}\right.$, $\left.\ldots, R_{m}\right\}$, the simulation of a $\left(\mathcal{A}_{s t}, \mathcal{A}_{t}\right)$-generic $k$-ary computable query on input $\$$ is broken up into three steps:

(i) The encoding step: The database $\$$ is encoded as a tuple of natural numbers $\left(N_{R_{1}}, \ldots, N_{R_{m}}\right)$, one for each relation of the database. Here, $N_{R_{i}}=$ Encode $\left(s_{i}\right)$, where $\left(s_{1}, \ldots, s_{m}\right)$ are the string representation of the quantifierfree formulas $\varphi_{\mathbf{C a n o n}_{\left(\mathcal{A}_{s t}, \mathcal{A}_{t}\right)}\left(R_{i}\right)}(i=1, \ldots, m)$ of the database $\operatorname{Canon}_{\left(\mathcal{A}_{s t}, \mathcal{A}_{t}\right)}(\$)$. It will be shown below that this encoding can be performed in the language $\mathrm{FO}(\boldsymbol{\Pi}, \sigma)+$ While. The set $\operatorname{Type}_{\left(\mathcal{A}_{s t}, \mathcal{A}_{t}\right)}(\mathbb{\$})$ is also computed, to be used in the decoding step.

(ii) The computing step: It was shown that the language $\mathrm{FO}(\Pi)+$ While has full computational power on the natural numbers, by simulating a counter machine [Gyssens et al. 1999].

More specifically, one can simulate a counter machine $M$ in $\mathrm{FO}(\boldsymbol{\Pi})+$ While such that on input $\left(N_{R_{1}}, \ldots, N_{R_{m}}\right), M$ halts if and only if $Q$ is defined on

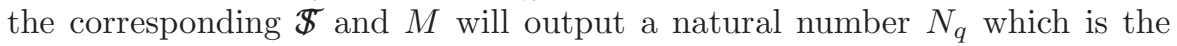
encoding of $Q(\$)$.

(iii) The decoding step: If $M$ terminates on input $\left(N_{R_{1}}, \ldots, N_{R_{m}}\right)$ then it outputs a natural number $N_{q}$. Using $\operatorname{Type}_{\left(\mathcal{A}_{s t}, \mathcal{A}_{t}\right)}(\$)$, the decoding algorithm computes the point set of which $N_{q}$ is the encoding. This can be implemented in the language $\mathrm{FO}(\Pi)+$ While.

We show next the details in the encoding and decoding algorithms that are different for $\left(\mathcal{A}_{s t}, \mathcal{A}_{t}\right)$-generic queries, as compared to the affine-generic queries considered in [Gyssens et al. 1999]. For ease of exposition, we will assume for the remainder of this proof that the input spatio-temporal database $\$$ has only one relation, with arity one, i.e., $\sigma=\{R\}$. For relations with arity greater than one, the encoding algorithm has to consider more variables. If the input database contains more relations, each relation has to be encoded separately. 
- The encoding algorithm can be expressed in $\mathrm{FO}(\Pi)$ + While. Roughly speaking, the encoding procedure enumerates all natural numbers and meanwhile stores the evaluation of the terms and formulas that are encoded by those numbers in relations that are called $T$ and $F$, respectively. This enumeration continues until one natural number is found that encodes a relation that is $\left(\mathcal{A}_{s t}, \mathcal{A}_{t}\right)$-isomorphic to $R$. This relation, for which the evaluation is stored in $F$, corresponds to $\operatorname{Canon}_{\left(\mathcal{A}_{s t}, \mathcal{A}_{t}\right)}(R)$. The set $\operatorname{Type}_{\left(\mathcal{A}_{s t}, \mathcal{A}_{t}\right)}(\$)$ is also computed, to use in the decoding step.

First, we explain the role of the relations $T$ and $F$ in more detail, as well as the way they are built during the encoding process.

The encoding program builds up terms and formulas until the formula is found that encodes $\operatorname{Canon}_{\left(\mathcal{A}_{s t}, \mathcal{A}_{t}\right)}(\$)$. The terms and formulas are stored in the relations $T$ and $F$. In general, the arity of $T$ is $(n+1)+2+2+l \times(n+1)$, where $n$ is the underlying dimension and $l=\operatorname{ar}(R)$. Under the assumption that $\operatorname{ar}(R)=1$ and the underlying dimension is 2 , each tuple in $T$ is of the form

$$
\left(u_{t_{O}}, u_{t_{E}}, u_{0}, u_{1}, u_{2}, u_{t}, p_{1}, p_{2}, p_{t}, v\right),
$$

where $\left(u_{t_{O}}, u_{t_{E}}\right)$ is a temporal coordinate system, $\left(u_{0}, u_{1}, u_{2}\right)$ a spatial coordinate system, $u_{t}$ the encoding of a term which only uses the variables $x_{1}, x_{2}, x_{t}$ (which are translated into $v_{1}, v_{2}, v_{t}$ ), and $v$ the value of the term when evaluated under the valuation $v_{1} \mapsto p_{1}, v_{2} \mapsto p_{2}, v_{t} \mapsto p_{t}$. The arity of $F$ is $(n+1)+2+1+l \times(n+1)$. Under the same assumptions, each tuple in $F$ is of the form

$$
\left(u_{t_{O}}, u_{t_{E}}, u_{0}, u_{1}, u_{2}, u_{f}, p_{1}, p_{2}, p_{t}\right),
$$

where $\left(u_{t_{O}}, u_{t_{E}}\right)$ and $\left(u_{0}, u_{1}, u_{2}\right)$ are as before, $u_{f}$ the encoding of a formula $\bar{\varphi}$ which only uses the variables $x_{1}, x_{2}, x_{t}$, and where $\varphi\left(p_{1}, p_{2}, p_{t}\right)$ is true.

In Figure 3, we give the structure of the encoding program in $\mathrm{FO}\left[\right.$ Between $^{n}$, Before, $\left.\mathbf{E q C R}^{\text {st }}\right\}$ ] +While. In this algorithm, it is assumed that substrings $s^{\prime}$ of a string $s$ is encountered in the enumeration before $s$ is encountered.

We will discuss in detail

(i) the representation of natural numbers (as we only have point-variables),

(ii) the expression that checks whether a certain natural number encodes a formula which represents $\operatorname{Canon}_{\left(\mathcal{A}_{s t}, \mathcal{A}_{t}\right)}(\Phi)$, and

(iii) the computation of the set $\operatorname{Type}_{\left(\mathcal{A}_{s t}, \mathcal{A}_{t}\right)}(R)$.

All other elements of the encoding can be adopted from the proof of [Gyssens et al. 1999] with only slight modifications. For ease of exposition, we give the formulas for $n=2$.

(i) Natural numbers can be represented by $(n+1)$-dimensional points using the computation plane technique introduced in Section 4. Further on, in de encoding and decoding algorithm, we need to simulate assignments such as $m:=0$ and $m:=m+1$ (since we have to run through all natural numbers in those algorithms). As an illustration, we explain here how thet are simulated in $\mathrm{FO}(\Pi)+$ While.

The expression $m:=0$, for example, is translated in $\mathrm{FO}(\Pi)+$ While by assigning to a spatio-temporal relation a point that is the origin of the chosen computation plane. The translated expression is

ACM Journal Name, Vol. V, No. N, 20 YY. 


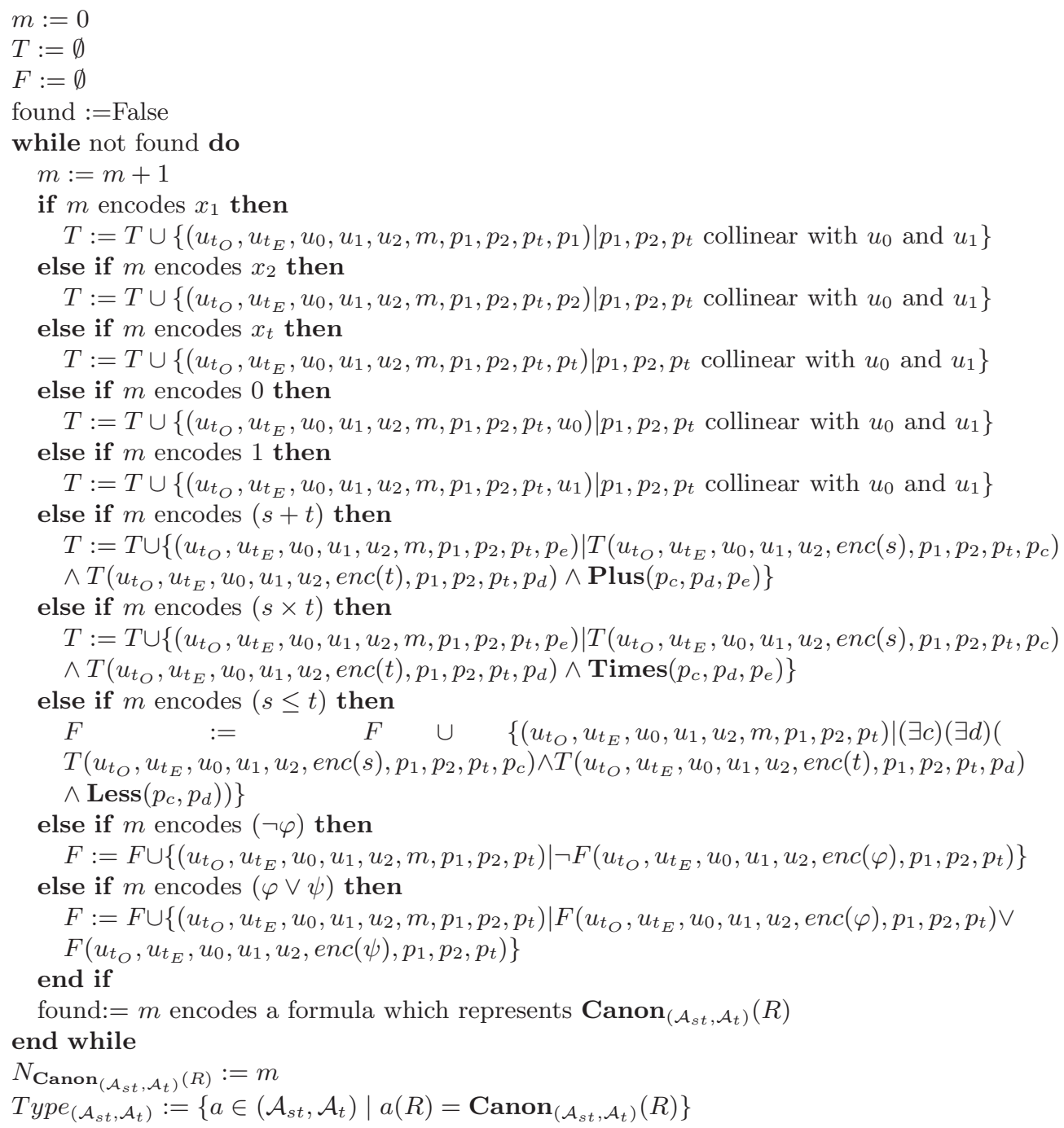

Fig. 3. The encoding program. The input is a $\mathrm{FO}(+, \times,<, 0,1, \bar{R})$-sentence.

$$
\begin{aligned}
N:=\left\{\left(u_{t_{O}}, u_{t_{E}}, u_{0}, u_{1}, u_{2}, v\right) \mid \mathbf{T C o S y s}_{\mathcal{A}}\left(u_{t_{O}}, u_{t_{E}}\right) \wedge\right. \\
\left.\operatorname{CoSys}_{\mathcal{A}}^{n}\left(u_{0}, u_{1}, u_{2}\right) \wedge \operatorname{Collinear}\left(u_{0}, u_{1}, v\right) \wedge v=u_{0}\right\} .
\end{aligned}
$$

For the assignment $m:=m+1$, we have:

$$
\begin{aligned}
N:=\left\{\left(u_{t_{O}}, u_{t_{E}}, u_{0}, u_{1}, u_{2}, v\right) \mid\right. & (\exists w)\left(N\left(u_{t_{O}}, u_{t_{E}}, u_{0}, u_{1}, u_{2}, w\right)\right. \\
& \left.\left.\wedge \operatorname{Plus}\left(u_{t_{O}}, u_{t_{E}}, u_{0}, u_{1}, u_{2}, w, u_{1}, v\right)\right)\right\} .
\end{aligned}
$$

The predicate Plus, which expresses that, relative to a computation plane, a certain point represents the sum of two other points can be written in $\mathrm{FO}(\boldsymbol{\Pi})$ 
+While because of Theorem 4.6.

(ii) We now give the expression $\varphi$ that checks whether a certain natural number $m$ encodes a formula which represents $\varphi_{\mathbf{C a n o n}_{\left(\mathcal{A}_{s t}, \mathcal{A}_{t}\right)}\left(R_{i}\right)}(i=1, \ldots, m)$. Remember that the evaluation of the formula encoded by $m$ is stored in the relation $F$. This relation has arity $n+3+K$, where $K$ is the maximal arity in the input database schema. Let $\left(p_{1}, p_{2}, \ldots, p_{n+3+K}\right)$ be a tuple of points satisfying $F$. The points $p_{1}$ and $p_{2}$ are a temporal coordinate system, $p_{n+3}$ represents the natural number $m$ encoding the formula and $p_{3}, p_{4}, \ldots, p_{n+2}$ form a hyperplane of which the plane through $p_{3}, p_{4}$ and $p_{5}$ will be use as a computation plane. The last $K$ points are the translation of the free variables in the formula encoded by $m$.

Let the formula $\psi_{\left(\mathcal{A}_{s t}, \mathcal{A}_{t}\right)}$ be the translation of the formula $\varphi_{\left(\mathcal{A}_{s t}, \mathcal{A}_{t}\right)}$ from Example 5.1. Intuitively, the next formula checks, for a natural number $m$, whether there exists an element of the group $\left(\mathcal{A}_{s t}, \mathcal{A}_{t}\right)$ that maps each point in $R$ to a point in the set of points satisfying the formula encoded by $m$, the evaluation of which is stored in $F$.

The following formula $\psi$ checks whether the right quantifier-free formula has been found. It reflects the stop condition of the While-loop that runs through the natural numbers. This formula $\psi$ can be written as

$$
\begin{aligned}
& \left(\forall u_{t_{O}}\right)\left(\forall u_{t_{E}}\right)\left(\forall u_{0}\right)\left(\forall u_{1}\right)\left(\forall u_{2}\right)\left(\mathbf{T C o S y s}_{\mathcal{A}}\left(u_{t_{O}}, u_{t_{E}}\right) \wedge\right. \\
& \left.\operatorname{CoSys}_{\mathcal{A}}^{n}\left(u_{0}, u_{1}, u_{2}\right)\right) \rightarrow\left(\exists v_{\alpha}\right)\left(\exists v_{\beta}\right)(\exists w)\left(\forall u_{t}\right)\left(\exists v_{a_{1,1}}\right)\left(\exists v_{a_{1,2}}\right)\left(\exists v_{a_{2,1}}\right)\left(\exists v_{a_{2,2}}\right) \\
& \left(\exists v_{b_{1}}\right)\left(\exists v_{b_{2}}\right)\left(N\left(u_{t_{O}}, u_{t_{E}}, u_{0}, u_{1}, u_{2}, w\right) \wedge\right. \\
& \left(\forall v_{x}\right)\left(\forall v_{y}\right)\left(\forall v_{t}\right)\left(F\left(u_{t_{O}}, u_{t_{E}}, u_{0}, u_{1}, u_{2}, w, v_{x}, v_{y}, v_{t}\right) \leftrightarrow\right. \\
& (\exists v)\left(\exists v_{x}^{\prime}\right)\left(\exists v_{y}^{\prime}\right)\left(R(v) \wedge \text { comp-coord }\left(u_{t_{O}}, u_{t_{E}}, u_{0}, u_{1}, u_{2}, v, v_{x}^{\prime}, v_{y}^{\prime}, u_{t}\right) \wedge\right. \\
& \psi_{\left(\mathcal{A}_{s t}, \mathcal{A}_{t}\right)}\left(u_{t_{O}}, u_{t_{E}}, u_{0}, u_{1}, u_{2}, v_{a_{1,1}}, v_{a_{1,2}}, v_{a_{2,1}}, v_{a_{2,2}}, v_{b_{1}}, v_{b_{2}}, u_{t}, v_{\alpha}, v_{\beta}\right. \text {, } \\
& \left.\left.\left.\left.v_{x}^{\prime}, v_{y}^{\prime}, u_{t}, v_{x}, v_{y}, v_{t}\right)\right)\right)\right) \text {. }
\end{aligned}
$$

In the above formula, we omitted, for all point variables except $v$, the sub formulas expressing collinearity with $v_{0}$ and $v_{1}$. Also, the predicate compcoord is an abbreviation for the fact that the translation of $v$ 's coordinates to the computation plane are $v_{x}^{\prime}, v_{y}^{\prime}$ and $u_{t}$. The exact formula expressing this can be found in the proof of Lemma 4.2, when the translation of relation predicates is explained.

(iii) For the set $\operatorname{Type}_{\left(\mathcal{A}_{s t}, \mathcal{A}_{t}\right)}(R)=\left\{\alpha \in\left(\mathcal{A}_{s t}, \mathcal{A}_{t}\right) \mid \alpha(R)=\operatorname{Canon}_{\left(\mathcal{A}_{s t}, \mathcal{A}_{t}\right)}(R)\right\}$, we compute two separate relations storing the $\mathcal{A}_{t}$-type, respectively $\mathcal{A}_{s t}$-type of the encoded relation. In the previous formula, it was checked whether there exists a transformation mapping all points in $R$ to points in the formula coded by $m$ (i.e., in $F$ ). Here, we compute that transformation: 


$$
\begin{aligned}
& T_{\mathcal{A}_{t}}:=\left\{\left(u_{t_{O}}, u_{t_{E}}, u_{0}, u_{1}, u_{2}, v_{\alpha}, v_{\beta}\right) \mid\left(\mathbf{T C o S y s}_{\mathcal{A}}\left(u_{t_{O}}, u_{t_{E}}\right) \wedge\right.\right. \\
& \left.\operatorname{CoSys}_{\mathcal{A}}^{n}\left(u_{0}, u_{1}, u_{2}\right)\right) \rightarrow(\exists w)\left(N\left(u_{t_{O}}, u_{t_{E}}, u_{0}, u_{1}, u_{2}, w\right) \wedge\right. \\
& \left(\forall v_{x}\right)\left(\forall v_{y}\right)\left(\forall v_{t}\right)\left(F\left(u_{t_{O}}, u_{t_{E}}, u_{0}, u_{1}, u_{2}, w, v_{x}, v_{y}, v_{t}\right) \leftrightarrow\right. \\
& (\exists v)\left(\exists v_{x}^{\prime}\right)\left(\exists v_{y}^{\prime}\right)\left(\exists v_{t}^{\prime}\right)\left(\exists v_{x}^{\prime \prime}\right)\left(\exists v_{y}^{\prime \prime}\right)\left(\exists v_{\alpha_{0,0}}\right)\left(\exists v_{\alpha_{0,1}}\right)\left(\exists v_{\alpha_{1,0}}\right)\left(\exists v_{\alpha_{1,1}}\right)\left(\exists v_{\beta_{0}}\right)\left(\exists v_{\beta_{1}}\right) \\
& \left(R(v) \wedge \text { comp-coord }\left(u_{t_{O}}, u_{t_{E}}, u_{0}, u_{1}, u_{2}, v, v_{x}^{\prime}, v_{y}^{\prime}, v_{t}^{\prime}\right) \wedge\right. \\
& \psi_{\mathcal{A}_{s t}}\left(u_{t_{O}}, u_{t_{E}}, u_{0}, u_{1}, u_{2}, v_{\alpha_{0,0}}, v_{\alpha_{0,1}}, v_{\alpha_{1,0}}, v_{\alpha_{1,1}}, v_{\beta_{0}}, v_{\beta_{1}},\right. \\
& \left.v_{t}^{\prime}, v_{x}^{\prime}, v_{y}^{\prime}, v_{t}^{\prime}, v_{x}^{\prime \prime}, v_{y}^{\prime \prime}, v_{t}^{\prime}\right) \wedge \\
& \left.\left.\left.\left.\psi_{\mathcal{A}_{t}}\left(u_{t_{O}}, u_{t_{E}}, u_{0}, u_{1}, u_{2}, v_{\alpha}, v_{\beta}, v_{x}^{\prime \prime}, v_{y}^{\prime \prime}, v_{t}^{\prime}, v_{x}, v_{y}, v_{t}\right)\right)\right)\right)\right\}
\end{aligned}
$$

and

$$
\begin{aligned}
& T_{\mathcal{A}_{s t}}:=\left\{\left(u_{t_{O}}, u_{t_{E}}, u_{0}, u_{1}, u_{2}, v_{\alpha_{0,0}}, v_{\alpha_{0,0}}, v_{\alpha_{0,1}}, v_{\alpha_{1,0}}, v_{\alpha_{1,1}}, v_{\beta_{0}}, v_{\beta_{1}}, u_{t}\right) \mid\right. \\
& \left(\operatorname{TCoSys}_{\mathcal{A}}\left(u_{t_{O}}, u_{t_{E}}\right) \wedge \operatorname{CoSys}_{\mathcal{A}}^{n}\left(u_{0}, u_{1}, u_{2}\right)\right) \rightarrow \\
& (\exists w)\left(N\left(u_{t_{O}}, u_{t_{E}}, u_{0}, u_{1}, u_{2}, w\right) \wedge\left(\forall v_{x}\right)\left(\forall v_{y}\right)\left(\forall v_{t}\right)(\right. \\
& F\left(u_{t_{O}}, u_{t_{E}}, u_{0}, u_{1}, u_{2}, w, v_{x}, v_{y}, v_{t}\right) \leftrightarrow(\exists v)\left(\exists v_{x}^{\prime}\right)\left(\exists v_{y}^{\prime}\right)\left(\exists v_{t}^{\prime}\right)\left(\exists v_{\alpha}\right)\left(\exists v_{\beta}\right) \\
& \left(R(v) \wedge \text { comp-coord }\left(u_{t_{O}}, u_{t_{E}}, u_{0}, u_{1}, u_{2}, v, v_{x}^{\prime}, v_{y}^{\prime}, v_{t}^{\prime}\right) \wedge\right. \\
& T_{\mathcal{A}_{t}}\left(u_{t_{O}}, u_{t_{E}}, u_{0}, u_{1}, u_{2}, v_{\alpha}, v_{\beta}\right) \wedge \\
& \psi_{\mathcal{A}_{t}}\left(u_{t_{O}}, u_{t_{E}}, u_{0}, u_{1}, u_{2}, v_{\alpha}, v_{\beta}, v_{x}^{\prime}, v_{y}^{\prime}, v_{t}^{\prime}, v_{x}^{\prime}, v_{y}^{\prime}, v_{t}\right) \wedge \\
& \psi_{\mathcal{A}_{s t}}\left(u_{t_{O}}, u_{t_{E}}, u_{0}, u_{1}, u_{2}, v_{\alpha_{0,0}}, v_{\alpha_{0,1}}, v_{\alpha_{1,0}}, v_{\alpha_{1,1}}, v_{\beta_{0}}, v_{\beta_{1}},\right. \\
& \left.\left.\left.\left.\left.v_{t}, v_{x}^{\prime}, v_{y}^{\prime}, v_{t}, v_{x}, v_{y}, v_{t}\right)\right)\right)\right)\right\} .
\end{aligned}
$$

- The decoding algorithm can be expressed in $\mathrm{FO}(\boldsymbol{\Pi})+$ While. Input databases are encoded by natural numbers. A counter machine simulates the query on this natural number and returns a natural number that encodes the output.

In the decoding algorithm, again all natural numbers are enumerated and the evaluation of the terms and formulas they encode are stored in relations called $T$ and $F$. When the number that is the output of the counter machine is encountered, the relation $F$ contains all points of the result, up to the transformation stored in $\operatorname{Type}_{\left(\mathcal{A}_{s t}, \mathcal{A}_{t}\right)}$ (because the query is $\left(\mathcal{A}_{s t}, \mathcal{A}_{t}\right)$-generic). The result corresponds to the set $Q\left(\operatorname{Canon}_{\left(\mathcal{A}_{s t}, \mathcal{A}_{t}\right)}(\mathbb{\Phi})\right)$. As $Q$ is assumed to be a $\left(\mathcal{A}_{s t}, \mathcal{A}_{t}\right)$-generic query, we have that for all $f \in \operatorname{Type}_{\left(\mathcal{A}_{s t}, \mathcal{A}_{t}\right)}(\mathbb{\$})$

$$
Q\left(\operatorname{Canon}_{\left(\mathcal{A}_{s t}, \mathcal{A}_{t}\right)}(\Phi)\right)=Q(f(\Phi))=f(Q(\Phi)),
$$

so $Q(\$)$ is computed as

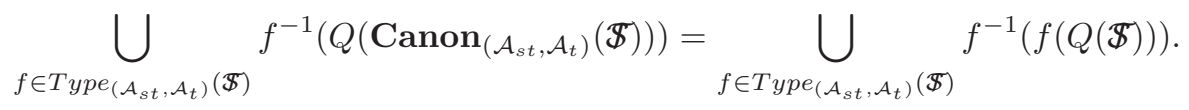

For completeness, we give a program Decode that, when applied to the encoding $N_{\varphi}$ of a formula $\varphi$, computes in a relation variable Dec the spatio-temporal relation defined by $\varphi$. Thereto it suffices to modify the encode program as shown in Figure 5.3. 


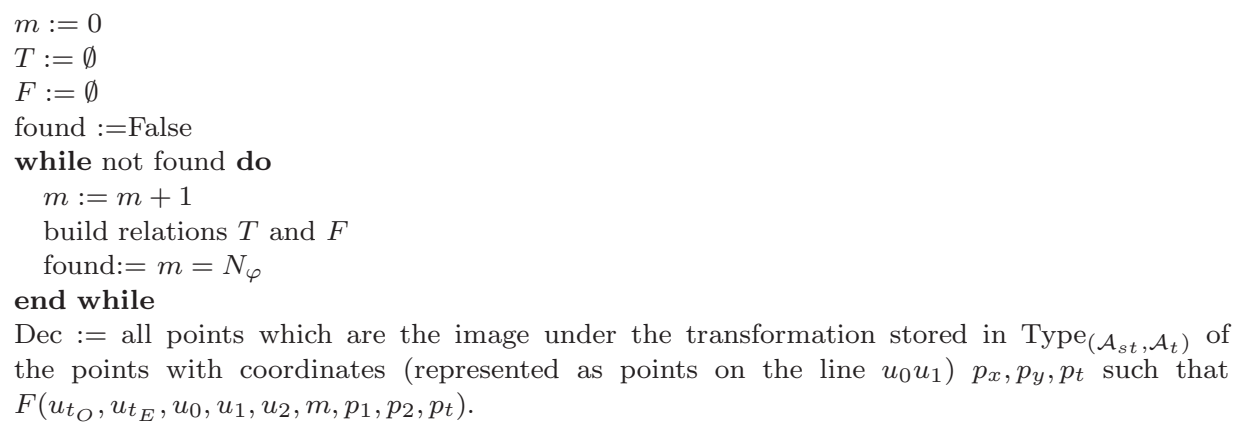

Fig. 4. The decoding program. The input is a natural number encoding a relation.

The formula constructing the output, using the above, only differs slightly from the formulas we gave when explaining the encoding algorithm. In the encoding phase, it had to be checked, for some natural number $m$, whether there existed a transformation mapping all points of $R$ to the points satisfying the formula encoded by $m$. Also, that transformation was computed. Here, we have the transformation stored in $\operatorname{Type}_{\left(\mathcal{A}_{s t}, \mathcal{A}_{t}\right)}$, and we know we have the right natural number $m$, so all points mapped by the transformation in $\operatorname{Type}_{\left(\mathcal{A}_{s t}, \mathcal{A}_{t}\right)}$ to points satisfying the formula encoded by $m$, are returned.

To conclude we summarize the conversion procedure. Given a $k$-ary computable query Q over a schema $\sigma=\left\{R_{1}, \ldots, R_{m}\right\}$, there exists a counter program $M$ such

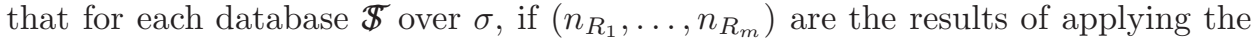
program Encode to $\mathbb{\$}$ then $M\left(n_{R_{1}}, \ldots, n_{R_{m}}\right)$ is the encoding of the quantifier-free formula defining $Q(\mathbb{\$})$, using the variables $x_{1}^{1}, \ldots, x_{1}^{n+1}, \ldots, x_{K}^{1}, \ldots, x_{K}^{n+1}$. If $Q(\mathbb{\$})$ is not defined, then $\mathrm{M}$ does not halt on this input. As already noted above, we can simulate $M$ by a program $P$ in $\mathrm{FO}(\boldsymbol{\Pi})+$ While. Hence, the query $\mathrm{Q}$ is expressed by the program

Encode;

$P$

Decode;

The reason that the problem of identifying sound and complete languages for the groups $\mathcal{F}_{s t}^{f}$ is still open, is that for those groups, there is no first-order logic formula expressing their graph. Indeed, it is not possible to express that there should exist a finite number of time moments for which there is a different affinity, when describing the groups $\mathcal{F}_{s t}^{f}$. Hence, we cannot use the above proof technique.

The previous theorem has a number of corollaries.

TheOREM 5.3. Let $\sigma$ be a database schema. Let $\left(\mathcal{F}_{s t}, \mathcal{F}_{t}\right)$ be one of the groups $\left(\mathcal{A}_{s t}, \mathcal{A}_{t}\right),\left(\mathcal{A}_{s t}, \mathcal{I}_{t}\right),\left(\mathcal{A}_{s t}, I d_{t}\right),\left(\mathcal{S}_{s t}, \mathcal{F}_{t}\right),\left(\mathcal{I}_{s t}, \mathcal{F}_{t}\right)$, or $\left(\mathcal{T}_{s t}, \mathcal{F}_{t}\right)$ with $\mathcal{F}_{t} \in\left\{\mathcal{A}_{t}, \mathcal{T}_{t}\right.$, $\left.I d_{t}\right\}$ and let $\Pi\left(\mathcal{F}_{s t}, \mathcal{F}_{t}\right)$ be as in Table III. The point language $\mathrm{FO}\left(\Pi\left(\mathcal{F}_{s t}, \mathcal{F}_{t}\right), \sigma\right)+$ While is sound and complete for the $\left(\mathcal{F}_{s t}, \mathcal{F}_{t}\right)$-generic computable spatio-temporal queries over $\bar{\sigma}$.

ACM Journal Name, Vol. V, No. N, $20 Y$ Y. 
Proof. The proof of this corollary is similar to the proof of Theorem 5.2. The encoding and decoding programs for the various transformation groups only differ where the transformation in $\operatorname{Type}_{\left(\mathcal{A}_{s t}, \mathcal{A}_{t}\right)}$ is described, and where a coordinate system needs to be defined. The rest of the proof is the same, regardless of the transformation groups considered. The descriptions of the coordinate systems for the various transformation groups can be found in the proof of Corollary 4.1

\section{CONCLUSION AND DISCUSSION}

We have investigated different genericity classes relative to the constraint database model for spatio-temporal databases and we have identified sound and complete languages for the $\mathrm{FO}(+, \times,<, 0,1, \bar{\sigma})$, respectively the computable, queries in (most of) these genericity classes. Some results were obtained by techniques introduced by Gyssens, Van den Bussche and Van Gucht [Gyssens et al. 1999], but for timedependent transformations we have introduced new proof techniques.

For what concerns computationally complete languages these techniques seem to be insufficient to deal with the genericity notions that are expressed by the groups $\left(\mathcal{A}_{s t}^{f}, \mathcal{A}_{t}\right),\left(\mathcal{A}_{s t}^{f}, \mathcal{I}_{t}\right),\left(\mathcal{A}_{s t}^{f}, I d_{t}\right),\left(\mathcal{S}_{s t}^{f}, \mathcal{F}_{t}\right),\left(\mathcal{I}_{s t}^{f}, \mathcal{F}_{t}\right)$, and $\left(\mathcal{T}_{s t}^{f}, \mathcal{F}_{t}\right)$ with $\mathcal{F}_{t} \in$ $\left\{\mathcal{A}_{t}, \mathcal{T}_{t}, I d_{t}\right\}$. The problem in adapting the proof technique of Theorem 5.2 to these groups is that it is not clear how we can express in the respective point-based logics that two spatio-temporal databases can be mapped to each other by some piecewise constant affinity. Indeed, since the number of pieces is not defined a priori, this might not be expressible. This would imply that yet another new proof technique would be required to deal with the remaining cases.

\section{REFERENCES}

Abel, D. And OoI, B. C., Eds. 1993. Advances in spatial databases-3rd Symposium SSD'g3. Lecture Notes in Computer Science, vol. 692. Springer-Verlag.

Allen, J. F. 1983. Maintaining knowledge about temporal intervals. Communications of the ACM 26, 11, 832-843.

Allen, J. F. And Ferguson, G. 1994. Actions and events in interval temporal logic. Journal of Logic and Computation 4, 5, 531-579.

Bochnak, J., Coste, M., And Roy, M. 1987. Géométrie Algébrique Réelle. Springer-Verlag, Berlin.

Böhlen, M. H., Jensen, C. S., And Scholl, M., Eds. 1999. Proceedings of the International Workshop on Spatio Temporal Database Management (STDBM'99). Lecture Notes in Computer Science, vol. 1678. Springer-Verlag.

Buchmann, A., Ed. 1989. Design and implementation of large spatial databases-1st Symposium SSD'89. Lecture Notes in Computer Science, vol. 409. Springer-Verlag.

Chandra, A. K. And Harel, D. 1980. Computable queries for relational data bases. Journal of Computer and System Sciences 21, 2, 156-178.

Chen, C. X. And ZAniolo, C. 2000. SQLST: A spatio-temporal data model and query language. In Conceptual Modeling, 19th International Conference on Conceptual Modeling (ER'00), V. C. S. A. H. F. Laender, S. W. Liddle, Ed. Lecture Notes in Computer Science, vol. 1920. Springer-Verlag, 96-111.

Chomicki, J. And Revesz, P. 1997. Constraint-based interoperability of spatio-temporal databases. In Proceedings of the 5th International Symposium on Advances in Spatial Databases. Lecture Notes in Computer Science, vol. 1262. Springer, 142-161.

Chomicki, J. And Revesz, P. 1999. A geometric framework for specifying spatiotemporal objects. In Proceedings of the 6th International Workshop on Temporal Representation and Reasoning. IEEE Computer Society, 41-46. 
Desloge, E. 1982. Classical Mechanics. Wiley, New York.

Egenhofer, M. J. And Herring, J. R., Eds. 1995. Advances in Spatial Databases-4th Symposium SSD'95. Lecture Notes in Computer Science, vol. 951. Springer-Verlag.

Erwig, M., Güting, R. H., Schneider, M., and Vazirgiannis, M. 1999. Spatio-temporal data types: An approach to modeling and querying moving objects in databases. GeoInformatica 3, 3, 269-296.

Frank, A., Grumbach, S., Güting, R., Jensen, C., Koubarakis, M., Lorentzos, N., Manopoulos, Y., Nardelli, E., Pernici, B., Schek, H.-J., Scholl, M., Sellis, T., Theodoulidis, B., And Widmayer, P. 1999. Chorochronos: A research network for spatiotemporal database systems. SIGMOD Record 28, 12-21.

Geerts, F., Haesevoets, S., And Kuijpers, B. 2001. A theory of spatio-temporal database queries. In Database Programming Languages, 8th International Workshop, DBPL 2001, Frascati, Italy, September 8-10, 2001, Revised Papers, G. Ghelli and G. Grahne, Eds. Lecture Notes in Computer Science, vol. 2397. Springer, 198-212.

Grumbach, S., Rigaux, P., And Segoufin, L. 1998. Spatio-temporal data handling with constraints. In Proceedings of the 6th international symposium on Advances in Geographic Information Systems (ACM-GIS'98), R. Laurini, K. Makki, and N. Pissinou, Eds. 106-111.

Grumbach, S. And Su, J. 1997. Queries with arithmetical constraints. Theor. Comput. Sci. 173, 1, $151-181$.

Gunther, O. And ScheK, H.-J., Eds. 1991. Advances in spatial databases-2nd Symposium $S S D^{\prime} 91$. Lecture Notes in Computer Science, vol. 525. Springer-Verlag.

Güting, R., Ed. 1999. Advances in Spatial Databases-6th Symposium SSD'99. Lecture Notes in Computer Science, vol. 1651. Springer-Verlag.

Güting, R. H., Bohlen, M. H., Erwig, M., Jensen, C. S., Lorentzos, N. A., Schneider, M., and Vazirgiannis, M. 2000. A foundation for representing and querying moving objects. $A C M$ Transactions on Databases Systems 25, 1-42.

Gyssens, M., Den Bussche, J. V., And Gucht, D. V. 1999. Complete geometric query languages. Journal of Computer and System Sciences 58, 3, 483-511.

Kanellakis, P. C., Kuper, G. M., and Revesz, P. 1990. Constraint query languages. In Proceedings of the 9th ACM Symposium on Principles of Database Systems (PODS'90). ACM Press, 299-313.

Kanellakis, P. C., Kuper, G. M., And Revesz, P. 1995. Constraint query languages. Journal of Computer and System Sciences 51, 26-52.

Kuijpers, B., Paredaens, J., And Gucht, D. V. 2000. Towards a theory of movie database queries. In Proceedings of the 7th International Workshop on Temporal Representation and Reasoning. IEEE Computer Society, 95-102.

Kuper, G. And Scholl, M. 2000. Geographic information systems. In Constraint databases, J. Paredaens, G. Kuper, and L. Libkin, Eds. Springer-Verlag, Chapter 2, 175-198.

LiBkin, L. 2000. Variable independence, quantifier elimination, and constraint representation. In Automata, Languages and Programming, 27th International Colloquium (ICALP 2000), U. Montanari, J. D. P. Rolim, and E. Welzl, Eds. Lecture Notes in Computer Science, vol. 1853. Springer-Verlag, 260-271.

Matijasevich, Y. V. 1993. Hilbert's Tenth Problem. MIT Press, Cambridge, MA.

Paredaens, J., Kuper, G., And Libkin, L., Eds. 2000. Constraint databases. Springer-Verlag.

Paredaens, J., Van den Bussche, J., And Van Gucht, D. 1994. Towards a theory of spatial database queries. In Proceedings of the 13th ACM Symposium on Principles of Database Systems. ACM Press, 279-288.

Pfoser, D. And Tryfona, N. 1998. Requirements, definitions and notations for spatiotemporal application environments. In Proceedings of the 6th International Symposium on Advances in Geographic Information Systems (ACM-GIS'98), R. Laurini, K. Makki, and N. Pissinou, Eds. 124-130.

Revesz, P. 2002. Introduction to Constraint Databases. Springer-Verlag.

Rigaux, P., Scholl, M., And Voisard, A. 2000. Introduction to Spatial Databases: Applications to GIS. Morgan Kaufmann.

ACM Journal Name, Vol. V, No. N, 20YY. 
Scholl, M. And Voisard, A., Eds. 1997. Advances in Spatial Databases-5th Symposium SSD'97. Lecture Notes in Computer Science, vol. 1262. Springer-Verlag.

Schwabhäuser, W., Szmielew, W., And Tarski, A. 1983. Metamathematische Methoden in der Geometrie. Springer-Verlag.

Sistla, A. P., Wolfson, O., Chamberlain, S., And DaO, S. 1997. Modeling and querying moving objects. In ICDE '97: Proceedings of the Thirteenth International Conference on Data Engineering. IEEE Computer Society, 422-432.

Van den Bussche, J. 2000. Constraint databases, queries and query languages. In Constraint databases, J. Paredaens, G. Kuper, and L. Libkin, Eds. Springer-Verlag, Chapter 2, 21-54. 